THE ASTROPHYSICAL JOURNAL SUPPLEMENT SERIES, 86:153-198, 1993 May

(c) 1993. The American Astronomical Society. All rights reserved. Printed in U.S.A.

\title{
OLD STELLAR POPULATIONS. IV. EMPIRICAL FITTING FUNCTIONS FOR FEATURES IN THE SPECTRA OF G AND K STARS ${ }^{1}$
}

\author{
Javier Gorgas, ${ }^{2}$ S. M. Faber, ${ }^{3}$ David Burstein, ${ }^{4}$ J. Jesús Gonzalez, ${ }^{3}$ Stéphane Courteau, ${ }^{5}$ AND Charles Prosser ${ }^{6}$ \\ Received 1991 June 24; accepted 1992 July 27
}

\begin{abstract}
Empirical fitting functions are presented that model the behavior of 11 strong atomic and molecular features in the spectra of Galactic $\mathrm{G}$ and $\mathrm{K}$ stars. The functions express the strength of spectral features as a function of $V-$ $K$, surface gravity, and metallicity. The gravity calibration rests on stars in Galactic globular and open clusters, for which gravities have been derived by fitting to stellar evolutionary isochrones.

The range of application of the fitting functions is set by the calibrating stars. There is good metallicity coverage from $[\mathrm{Fe} / \mathrm{H}]=-0.75$ to $+0.50 \mathrm{dex}$, and cluster ages span $3-15 \times 10^{9}$ yr. However, the range in age is large only near solar metallicity, and the gravity partial derivatives may therefore be uncertain at metallicities much higher or lower than this value. In addition, the fitting functions build in whatever abundance ratio trajectories of elements relative to iron are present in local Galactic stars; these might differ from the element ratios present in external galaxies.

In general, the behavior of the fitting functions matches expectations as to how these spectral features should behave as a function of basic stellar atmospheric parameters. The exception is CN ( $4150 \AA$ ), for which we find strong evidence for rapid onset of giant-branch mixing of $\mathrm{CNO}$ products to the stellar surface, setting in at $V-K=$ 2.10. This confirms other recent studies of this index.

The paper also presents new Lick line strength data on 112 field dwarfs and 92 cluster stars, plus colors, metallicities, and surface gravities for all Lick $\mathrm{G}$ and $\mathrm{K}$ stars published previously. The fitting functions are inverted to produce new output gravity and metallicity estimates for these stars and new average metallicities for all clusters, with errors of $\pm 0.15 \mathrm{dex}$ in $[\mathrm{Fe} / \mathrm{H}]$ and $\pm 0.23 \mathrm{dex}$ in $\log g$ per star.
\end{abstract}

Subject headings: Galaxy: stellar content — globular clusters: general — stars: abundances — stars: evolution

\section{INTRODUCTION}

This paper is the next step in a long-term program to understand the absorption-line spectra of elliptical galaxies as a function of age and metallicity. In previous papers, we have compared elliptical galaxy spectra to those of globular clusters (Burstein et al. 1984, hereafter Paper I), analyzed field K giant spectra (Faber et al. 1985, hereafter Paper II), and estimated the metallicity of globular clusters using an iron-line index (Burstein. Faber, \& Gonzalez, 1986, hereafter Paper III).

The present paper analyzes stellar spectra along the same lines as in Paper II, but with better fitting techniques and a larger sample of stars. The main product is a set of empirical "fitting functions" that express the strength of strong spectral features in cool stars as a function of surface temperature, surface gravity, and $[\mathrm{Fe} / \mathrm{H}]$. The features modeled are the 11 atomic and molecular features on the Lick system that have appeared in previous papers. The broadened stellar sample

\footnotetext{
${ }^{1}$ Lich Observatory Bulletin No. 1229.

${ }^{2}$ Departamento de Astrofisica, Facultad de Fisicas, Universitad Complutense, Madrid, E28040, Spain.

${ }^{3}$ UCO/Lick Observatory, Board of Studies in Astronomy and Astrophysics, University of California, Santa Cruz, CA 95064.

${ }_{4}^{4}$ Department of Physics and Astronomy, Arizona State University, Tempe, AZ 85287-1504.

${ }^{5}$ Department of Astronomy, 512 Space Sciences Building, Cornell University, Ithaca, NY 14853-6801.

${ }^{6}$ Center for Astrophysics, 60 Garden Street, Cambridge, MA 02138.
}

now includes field $\mathrm{G}$ dwarfs, plus stars from several open and globular clusters. The cluster stars remedy a serious lack in Paper II by providing a sample of giants and subgiants with known gravities, derived by fitting to cluster evolutionary tracks. Gravities of field $\mathrm{G}$ dwarfs have also been estimated from trigonometric parallaxes and spectroscopic indicators. The net result is a large sample of stars with known temperature, metallicity, and gravity. Least-squares fits have been used to derive empirical fitting functions that model each spectral feature as a function of these three atmospheric parameters.

The range of ages and metallicities covered by the fitting functions is determined by the range of those parameters covered by the stellar sample. The present functions can be used to cover $[\mathrm{Fe} / \mathrm{H}]$ from -0.75 to +0.50 dex and ages from 3 to $15 \times$ $10^{9} \mathrm{yr}$, with some limitations (see below). In parallel developments, stellar population modelers have recently begun to generate completely theoretical indices based on theoretical stellar evolutionary tracks and model stellar atmospheres (e.g., Buzzoni 1989, Gulati, Malagnini, \& Morossi 1990). The present fitting functions complement these programs, providing easyto-use empirical data with which to test the theoretical line strengths. This can also be done on a star-by-star basis, but fitting functions are a better approach because they average over the error and uncertainty of any one star.

Fitting functions also offer an important advantage over the traditional stellar library approach to computing integrated line strengths. With fitting functions, one can easily include any star within the age and metallicity ranges covered by the 
functions. This remedies a severe drawback of libraries, which for practical reasons usually do not contain stars over the full range of ages and metallicities needed. The fitting functions effectively allow us to interpolate smoothly over these missing stars.

On the other hand, the present calibrations also have two significant limitations. As there is no range in age among our calibrating stars at either very low or very high metallicities, gravity (and hence age) trends are less well determined at these extremes. The present fitting formulae also build in the wellknown element abundance-ratio trajectories, [X/Fe] versus $[\mathrm{Fe} / \mathrm{H}]$, that are present in local disk and halo stars (e.g., Wheeler, Sneden, \& Truran 1989). These trends might differ in external stellar populations, to which the present functions should therefore be applied with caution. A first study (Worthey, Faber, \& Gonzalez 1992) indeed indicates that Mg/ $\mathrm{Fe}$ is stronger in giant $\mathrm{E}$ galaxies than predicted by these functions.

In addition to providing a quantitative gravity calibration, we improve further on Paper II by using a unified calibration for all stars, which forces the calibration of the giants to merge smoothly with that of the dwarfs. In effect, the hot giants are "anchored" by the hotter dwarfs. This has increased the calibration accuracy for the hot giants and corrected some small errors that appeared in Paper II. With the much larger range of gravities available here, we also now discern certain gravity effects that were previously invisible in Paper II. We see, for example, that gravity modulates both $\mathrm{Fe} 5270$ and $\mathrm{Fe} 5335$, but the effects are small and are easily calibrated.

The present paper builds on a long series of earlier works on the low-resolution spectra of $\mathrm{G}$ and $\mathrm{K}$ stars (e.g., McClure \& van den Bergh 1968; Spinrad \& Taylor 1969; Dickow et al. 1970; Hansen \& Kjaergaard 1971; Gottlieb \& Bell 1971; Williams 1971a, b; Gustafsson, Kjaergaard, \& Anderson 1974; Mould \& McElroy 1978). The main difference in the present treatment is the inclusion of stars of known gravity, which allows us to solve explicitly for the gravity terms for the first time. This in turn makes it possible to follow the line-strength evolution of a given stellar population versus age, opening the way to more realistic galaxy models.

While preparing the present work for publication, we have also been collecting spectra of stars in four additional clusters as a further test of the fitting functions. In addition, we plan to add 10 new spectral features that we hope will yield an even more accurate abundance test for Fe-peak elements. Parallel measurements on 300 galaxy spectra are also in preparation. These new results and observations will be described in future papers.

This subject is unfortunately rather technical, requiring inclusion of many details on the handling of the data and the derivation and validity of the fits. We have tried to place most of this material in the footnotes to the tables and in the figure legends. The reader interested in obtaining a brief overview should scan the text starting at $\S 3$ and consult the figures for major results. The fits to the individual spectral features are discussed in $\S 4$ and are then inverted in $\S 5$ to derive output metallicities and gravities for all stars. This final step provides an important check on the accuracy of the fitting functions. The range of usefulness and the limitations of the current fitting functions are discussed in $\S 6$.

\section{OBSERVATIONS AND BASIC DATA}

\subsection{Spectra and Line Strengths}

The moderate-resolution spectra used here ( 9 A FWHM) were taken from 1972 to 1984 with the Cassegrain spectrograph and red-sensitive image-dissector scanner (IDS) (Robinson \& Wampler 1972) on the $3 \mathrm{~m}$ Shane telescope of Lick Observatory. The observing and data reduction procedures are described in detail in Papers I and II. The spectra are fairly homogeneous, having been taken with the same equipment and reduced using the same procedures. The line strengths used here are on the same system as Papers I-III.

The spectra cover approximately 4000-6200 $\AA$ and were reduced to a linear wavelength scale of $1.25 \AA$ per channel using helium, neon, mercury, and cadmium lamps. The equivalent-width system was monitored via nightly observations of standard $\mathrm{K}$ giant stars, and small run corrections were applied as necessary. Eleven absorption line indices were measured, as described in Paper II. The features and their associated spectral intervals are listed in Table 1.

RMS observational errors averaged over the whole sample are also given in Table 1 . The field $\mathrm{K}$ giants from Paper II have better than average data, and their errors are somewhat lower than quoted in Table 1. The data on the field G dwarfs, M67, and NGC 7789 are comparable to the field K giants. The data for the globular clusters and NCG 188 are of lower than average quality, especially those for the fainter stars. The errors for these groups are roughly 50\% larger than quoted in Table 1.

For very cool stars, the actual errors of measurement for certain features are larger than quoted in Table 1. The spectra of such stars are extremely jagged owing to deep molecular bands, and a slight misplacement in wavelength can result in large errors. The indices most affected are $\mathrm{H} \beta, \mathrm{Mg} b, \mathrm{Mg}_{1}$, $\mathrm{Mg}_{2}$, and $\mathrm{Na} \mathrm{D}$.

\subsection{Input Atmospheric Parameters}

When starting this program, we elected to use $V-K$, surface gravity, and metallicity $([\mathrm{Fe} / \mathrm{H}])$ as the basic atmospheric parameters. We chose the color index $V-K$ rather than $T_{e}$ in order to stay as close as possible to the observational domain and to remain independent of future revisions in the colortemperature scale. $V-K$ was recommended as being fairly insensitive to metallicity and surface gravity (Cohen, Frogel, \& Persson 1978). Since that time, Taylor, Johnson, \& Joner (1987) have restudied blanketing in various color indices and concluded from a semiempirical approach that $V-K$ is not in fact immune to metallicity effects. For solar metallicity $\mathrm{K}$ giants, they estimate that a change in $[\mathrm{Fe} / \mathrm{H}]$ of +0.5 dex reddens $V-K$ by 0.17 mag. However, Bell \& Gustafsson (1989) find much smaller changes of \pm 0.02 mag using theoretical stellar atmospheres and model flux distributions. Even newer models by Kurucz (1992) confirm this.

Such an effect, even of the magnitude found by Taylor et al., does not invalidate the present fitting-function approach since one can simply substitute the appropriate combination of metallicity and temperature for $V-K$ in each line-strength expression. However, it does affect the inverse process whereby a star's metallicity is inferred from a particular spectral feature. When the details are worked out, one finds that, if $V-K$ 
TABLE 1

WAVELENGTH INTERVALS AND RMS ERRORS

\begin{tabular}{|c|c|c|c|c|c|}
\hline Feature & Central Bandpass & Continuum Bandpass & $\sigma_{o b s}^{a}$ & $\sigma_{f i t}^{b}$ & Fit No. ${ }^{c}$ \\
\hline \multirow[t]{2}{*}{$\mathrm{CN}$} & $4144.00-4177.75$ & $4082.00-4118.25$ & $0.029 \mathrm{mag}$ & $0.030 \mathrm{mag}$ & 1 \\
\hline & & $4246.00-4284.75$ & $0.029 \mathrm{mag}$ & $0.050 \mathrm{mag}$ & 2 \\
\hline \multirow[t]{2}{*}{$G$ band } & $4283.25-4317.00$ & $4268.25-4283.25$ & $0.57 \AA$ & $0.64 \AA$ & 3 \\
\hline & & $4320.75-4335.75$ & $0.57 \AA$ & $0.58 \AA$ & 4 \\
\hline \multirow[t]{2}{*}{$\mathbf{H} \beta$} & $4849.50-4877.00$ & $4829.50-4848.25$ & $0.26 \AA$ & $0.28 \AA$ & 5 \\
\hline & & $4878.25-4892.00$ & & & \\
\hline \multirow[t]{2}{*}{$\mathrm{Mg}_{2}$} & $5156.00-5197.25$ & $4897.00-4958.25$ & $0.011 \mathrm{mag}$ & $0.023 \mathrm{mag}$ & 6 \\
\hline & & $5303.00-5366.75$ & & & \\
\hline \multirow[t]{2}{*}{$\mathrm{Mg}_{1}$} & $5071.00-5134.75$ & $4897.00-4958.25$ & $0.010 \mathrm{mag}$ & $0.017 \mathrm{mag}$ & 7 \\
\hline & & $5303.00-5366.75$ & & & \\
\hline \multirow[t]{2}{*}{$\mathrm{Mg} b$} & $5162.00-5193.25$ & $5144.50-5162.00$ & $0.32 \AA$ & $0.42 \AA$ & 8 \\
\hline & & $5193.25-5207.00$ & & & \\
\hline \multirow[t]{2}{*}{ Fe5270 } & $5248.00-5286.75$ & $5235.50-5249.25$ & $0.32 \AA$ & $0.36 \AA$ & 9 \\
\hline & & $5288.00-5319.25$ & & & \\
\hline \multirow[t]{2}{*}{ Fe5335 } & $5314.75-5353.50$ & $5307.25-5317.25$ & $0.35 \AA$ & $0.35 \AA$ & 10 \\
\hline & & $5356.00-5364.75$ & & & \\
\hline \multirow[t]{2}{*}{$\mathrm{Na} D$} & $5879.25-5910.50$ & $5863.00-5876.75$ & $0.34 \AA$ & $0.45 \AA$ & 11 \\
\hline & & $5924.50-5949.25$ & & & \\
\hline \multirow[t]{2}{*}{$\mathrm{TiO}_{1}$} & $5939.00-5995.25$ & $5819.00-5850.25$ & $0.009 \mathrm{mag}$ & $0.009 \mathrm{mag}$ & 12 \\
\hline & & $6041.00-6104.75$ & & & \\
\hline \multirow[t]{2}{*}{$\mathrm{TiO}_{2}$} & $6192.00-6273.25$ & $6069.00-6142.75$ & $0.011 \mathrm{mag}$ & $0.012 \mathrm{mag}$ & 13 \\
\hline & & $6375.00-6146.25$ & & & \\
\hline
\end{tabular}

${ }^{a} \sigma_{\text {obs }}$ is the estimated rms error of an average observation.

${ }^{b} \sigma_{\text {fit }}$ is the rms residual about the final fit.

${ }^{\mathrm{c}}$ Fit number in Table 6.

reddens with increasing metallicity, calibrating versus $V-K$ instead of $T_{e}$ magnifies the effect of observational error on the derived metallicity. This increase is largest for features that strengthen most rapidly with decreasing temperature. Using the estimated metallicity reddening by Taylor et al. together with the mean trend lines versus $V-K$ for K giants in Paper II, we find that the error in derived metallicity is increased by nearly $100 \%$ for $\mathrm{Mg}_{1}$ and $\mathrm{Mg}_{2}$, by $20-40 \%$ for $\mathrm{Mg} b, \mathrm{Na} \mathrm{D}, \mathrm{Fe}$ 5270 , and $\mathrm{Fe} 5335$, but is virtually unchanged for $\mathrm{CN}$.

On the other hand, the predicted effects from Bell \& Gustafsson are much smaller. Eventually the change in $V-K$ with abundance will have to be worked out before stellar population models can be computed. However, we do not need to deal with that problem here since the present paper remains exclusively within the $V-K$ domain.

Previous calibrations of gravity-sensitive indices have usually used absolute magnitude as the measure of stellar surface gravity. We prefer to use gravity directly, as this is the physical parameter that governs the atmosphere. Our primary input gravities come from cluster stars (fitted to evolutionary tracks) and from field dwarfs with measured trigonometric parallaxes. Gravities for a few field dwarfs come also from spectroscopic values, as discussed below.

\subsection{Three Stellar Data Samples \\ 2.3.1. Field K Giants}

The first stellar data set is the field $\mathrm{K}$ giant sample published in Paper II, containing 90 solar neighborhood field stars. Input values of $V-K$ and $[\mathrm{Fe} / \mathrm{H}]_{0}$ were taken from that paper, with $[\mathrm{Fe} / \mathrm{H}]_{0}$ revised slightly in a few cases. The feature strengths were taken unmodified from Paper II and are not repeated here. Gravities for the field $\mathrm{K}$ giants were estimated using $\mathrm{Mg}_{2}$, as explained in $\S 4.1$. Final input atmospheric parameters for the field $\mathrm{K}$ giants are described further there.

\subsubsection{Field Dwarfs}

The second set of stars is a new sample of 112 field dwarfs. These are mostly $F$ and $G$ types, but there is a sprinkling of stars down the main sequence to M7 V. Laird's (1985) dwarf sample was used as the basic finding list, to which a few stars 
were added. The metallicity estimates come mainly from Laird's Strömgren photometry, extended and amended by high-dispersion spectroscopic studies. The metallicity coverage is good among types $\mathrm{F}$ and $\mathrm{G}$ but falls off severely below $\mathrm{K} 0$ $\mathrm{V}$, where only a few abundance determinations are available. We have assumed solar abundance for stars without independent values. (New abundance estimates have been derived for many of these by inverting the fitting functions; see $\S 5$ below.)

Strömgren surface gravities have been measured by Laird, and gravities from trigonometric parallaxes and high-dispersion abundance studies are also available for many stars. We consider the trigonometric values to be fundamental and have preferred them in case of disagreement. We note a few discrepant Strömgren estimates for stars with $[\mathrm{Fe} / \mathrm{H}]$ below -0.55 dex and have not used any Strömgren values below this level. $V-K$ colors for the dwarfs were taken from the literature or interpolated from published values of $R-I$ (Johnson). All data for the field dwarf sample, including the new feature strengths and data sources for gravities and colors, are summarized in Table 2.

\subsubsection{Cluster Stars}

The third data set is summarized in Table 3 and contains stars from 10 clusters covering a variety of ages and metallicities. Some of these have appeared before in Papers II and III, but most are new here. The present list of clusters contains five old, metal-poor globulars, one intermediate-metallicity globular, and four nearly solar clusters ranging in age from 0.2 to 10 Gyr. The coverage in age (and therefore in gravity) is fairly good at the metal-rich end but poor at the metal-poor end. This means that gravity trends are not well established at low metallicities, but this is unavoidable given the present distribution of stellar ages and metallicities in the Galaxy.

Gravities were estimated by matching the location of a star in the H-R diagram to evolutionary tracks, as explained below. Adopted reddenings, distance moduli, ages, and photometry sources for the individual clusters are summarized in Table 4. Where available, we adopted VandenBerg's $(1983,1985)$ values in order to stay compatible with his tracks.

A composite $\mathrm{H}-\mathrm{R}$ diagram for all cluster stars is shown in Figure 1, and the individual cluster diagrams with adopted theoretical isochrones overlaid are shown in Figure 2. For the turnoff and subgiant regions (in M67 and NGC 188), we used the solar metallicity isochrones given by VandenBerg (1985) for ages 5 and $10 \mathrm{Gyr}$ (Figs. $2 a$ and $2 c$, solid lines). For giant branch stars, we used the Revised Yale Isochrones of Green, Demarque, \& King (1987), linearly interpolated in [ Fe/H] with $Y=0.25$. These are shown by the dotted lines in Figures $2 a-2 d$ and correspond to the assumed ages in Table 4. Yale isochrones for the most metal-poor clusters are shown in Figure $2 e$.

Gravities for the giant stars were estimated by comparing to Yale $M_{V}$, thus avoiding most of the uncertainties in the colortemperature calibration. We ignored any mismatch in $B-V$ in Figure 2, as this translates to less than $0.10 \mathrm{dex}$ in $\log g$ for all clusters except NGC 7789 and NGC 188 (see Appendix A). For M67 and NGC 188, we were also able to estimate gravities from the giant-branch isochrones of VandenBerg \& Laskarides (1987) (this required using $M_{\mathrm{bol}}$, with bolometric corrections taken from Cohen et al. 1978). The agreement between the two approaches was excellent, with an rms difference of only 0.03 dex in $\log g$. The adopted values are the average of both estimates.

Gravities for clump (horizontal-branch) stars in NGC 188, M67, and M71 were determined by interpolating in the solar metallicity ZAHB of VandenBerg (1985). These stars are indicated in Table 3. For M71, we checked these results using the zero-age theoretical horizontal branches of Sweigart (1987). Both sources agreed well, with an rms of $0.04 \mathrm{dex}$ in $\log g$. The latter source was also used for the HB star in M5. For HB stars, our deduced gravities average 0.20 dex less than for giantbranch stars at the same $M_{V}$. Gravities for AGB stars in M71, M13, and M92 were estimated by applying a correction in effective temperature and mass to the gravities of stars at the same $M_{V}$ on the giant branch (assuming $M_{\mathrm{AGB}}=0.65 M_{\odot}$ ).

Further discussion of the accuracies of the gravities and their dependence on the assumed evolutionary tracks is given in Appendix A. The conclusion is that random errors in $\log g$ are less than 0.20 dex for all stars, and systematic errors are comparable to or less than 0.10 dex for the sample as a whole.

Complete data on the cluster stars are given in Table 3. Many $V-K$ values are published, but some had to be interpolated from $B-V$. The scheme for doing this took abundance into account and is described in Appendix B. In the course of analyzing the line strengths, we discovered a handful of cluster stars that appeared to be nonmembers. These stars and the membership evidence on them are included in the notes to Table 3. They are neither plotted in Figure 2 nor used in what follows. The basic atmospheric input parameters $V-K, \log g$, and $[\mathrm{Fe} / \mathrm{H}]$ are summarized for all stars in Table 5.

\section{FITTING FUNCTIONS}

The aim in the present fits is to achieve a convenient, empirical fitting function for each index with as few free parameters as possible. We claim no physical justification for any of these fits-they are merely mathematical forms arrived at by trial and error. In all cases save one, the results are consistent with our preconceptions as to how these indices should behave with known changes in the atmospheric parameters. The one exception is $\mathrm{CN}$, for which the data strongly imply the onset of internal mixing and a change in atmospheric composition at the base of the giant branch ( see $\S 4.7$ ).

The present fitting functions are accurate only over the space spanned by the calibrating stars and should not be extrapolated much beyond this region. To show the limits graphically, Figure 3 shows the distribution of the sample in the (log $g, V-K$ ) plane. This diagram is important not only as a guide to the region of validity of the present functions but can also be used as a quick aid in estimating the gravities of cool stars with known $V-K$.

There are two other important limitations. As noted, the sample contains a fair spread in metallicity among the $\mathrm{F}$ and $\mathrm{G}$ dwarfs but a very weak selection below $\mathrm{K} 0 \mathrm{~V}$. The total range in metallicity among giants is also good, but, as metallicity tends to be correlated with location in the $(\log g, V-K)$ plane, the range in $Z$ at any one location in Figure 3 is smaller. The widest range in age (and thus $\log g$ ) is obtained near solar metallicity. The gravity partial derivatives in the fitting func- 


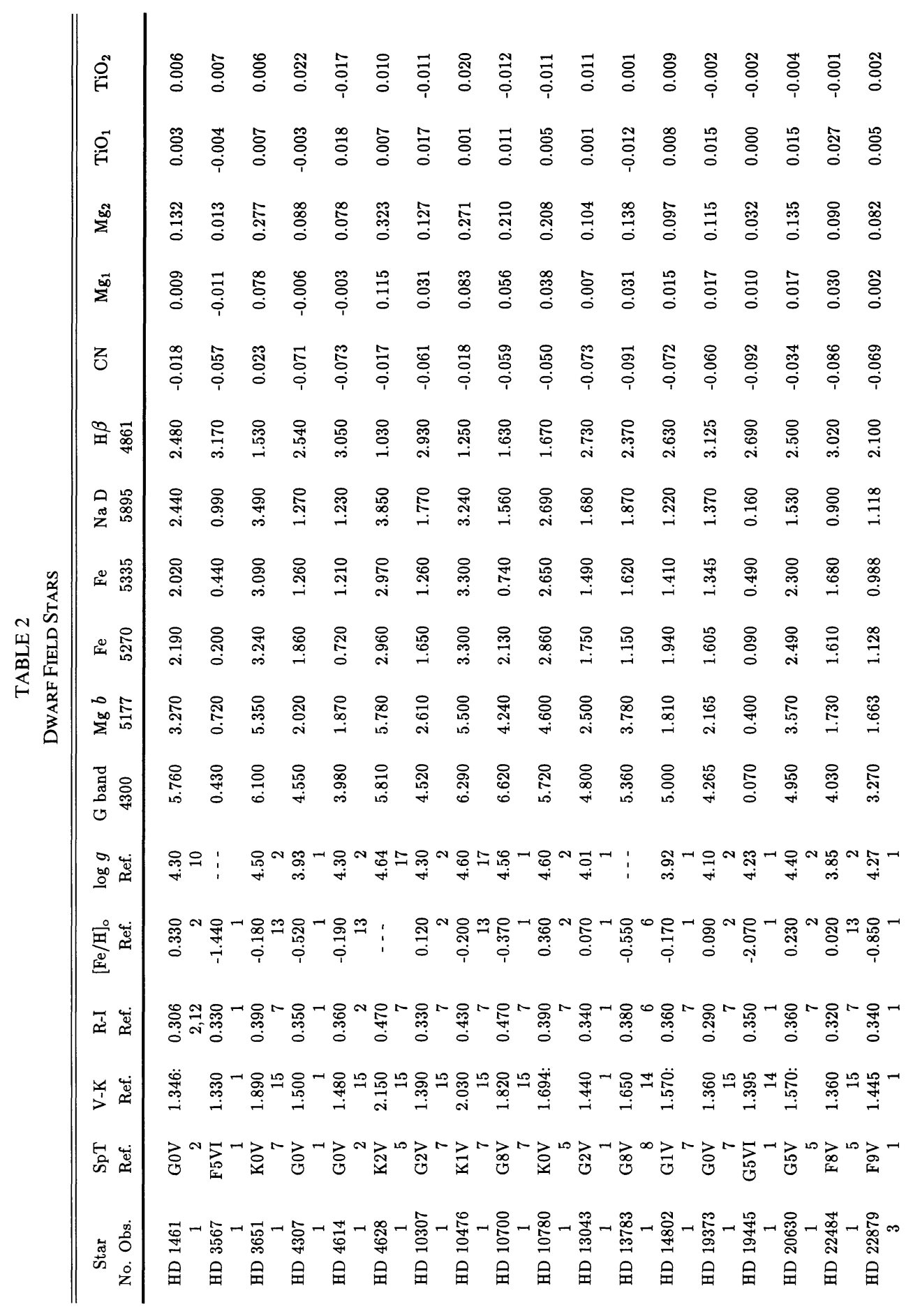




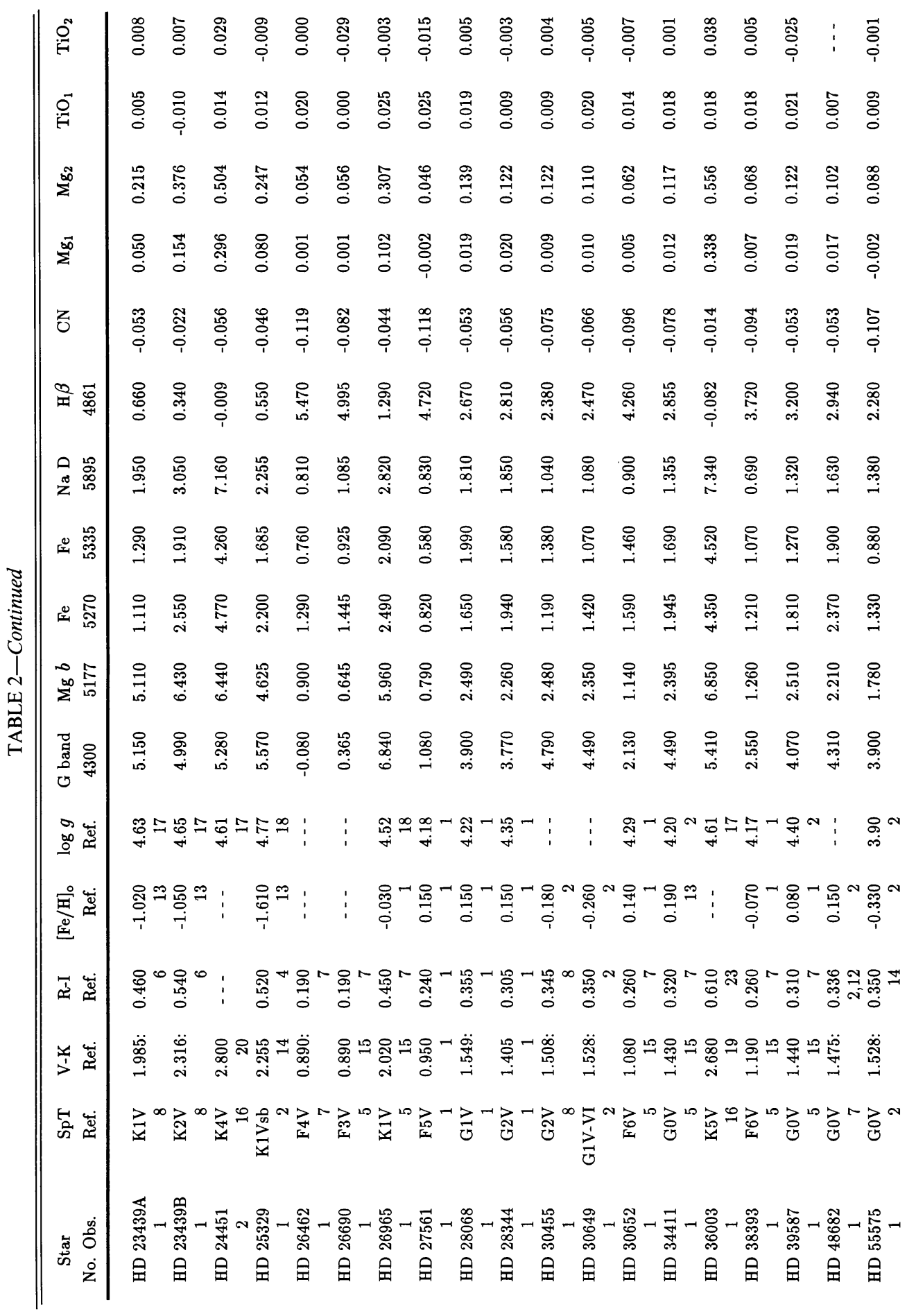




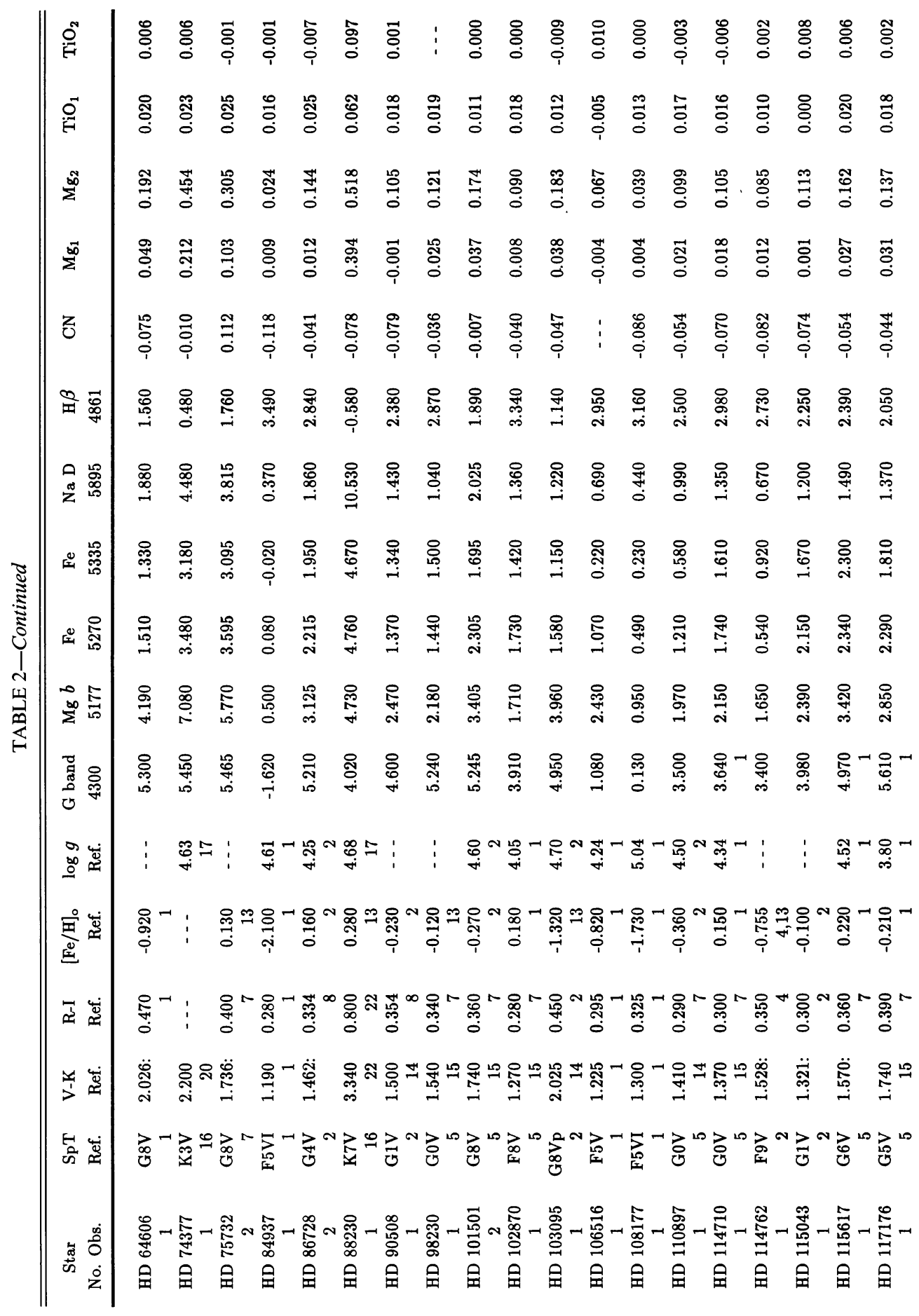




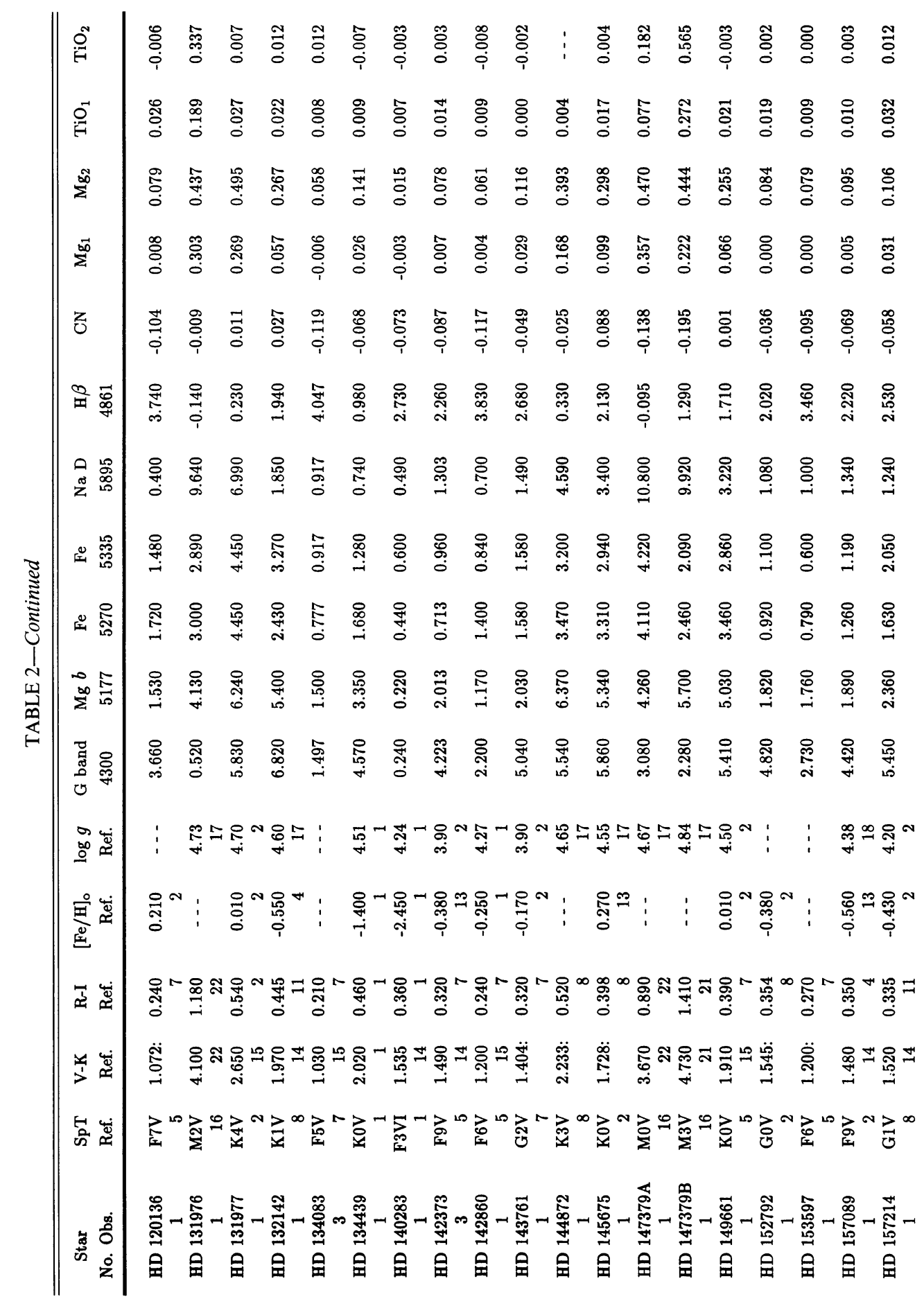




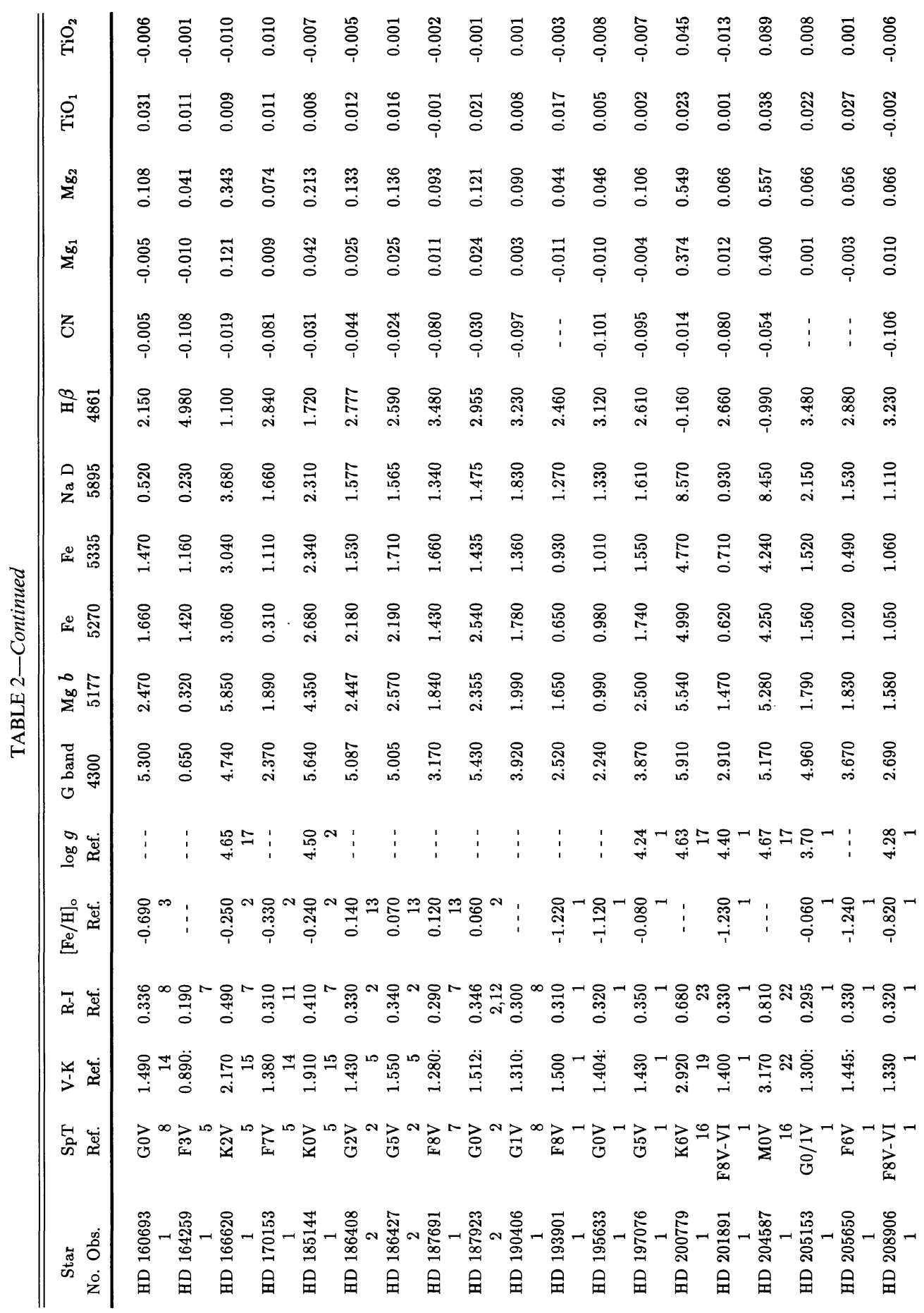




\begin{tabular}{|c|c|c|}
\hline ֻृ & 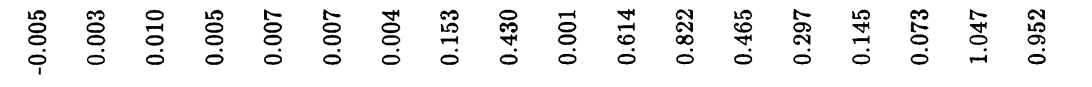 & \\
\hline$\stackrel{\circ}{\circ}$ & 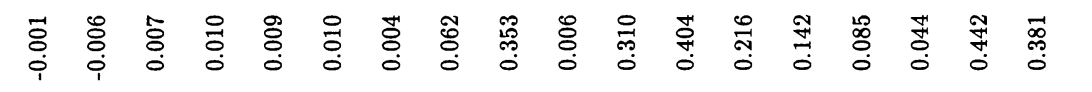 & \\
\hline 这 & 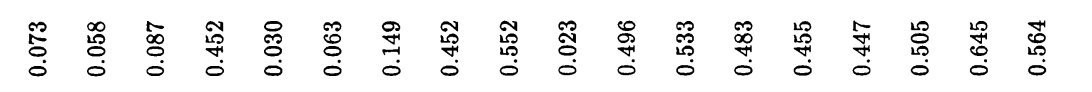 & \\
\hline 点 & 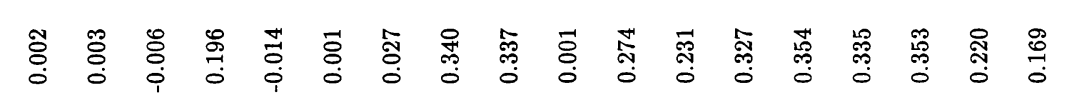 & \\
\hline z & 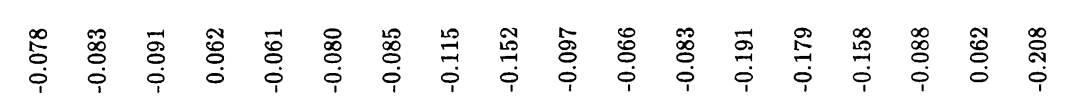 & \\
\hline 畕察 & 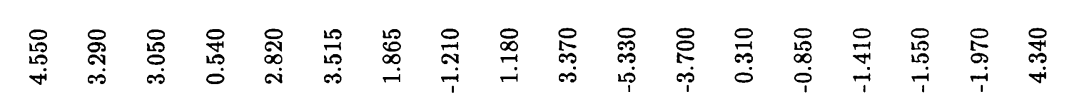 & \\
\hline 品密 & 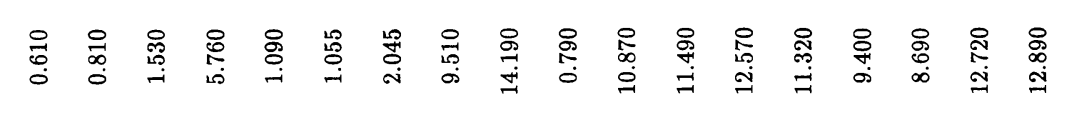 & \\
\hline 禺 & 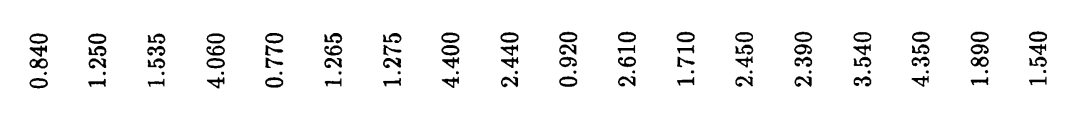 & \\
\hline 윰 & 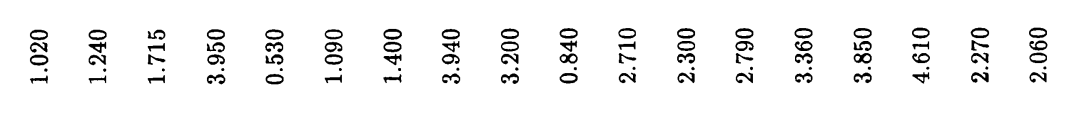 & \\
\hline 然言 & 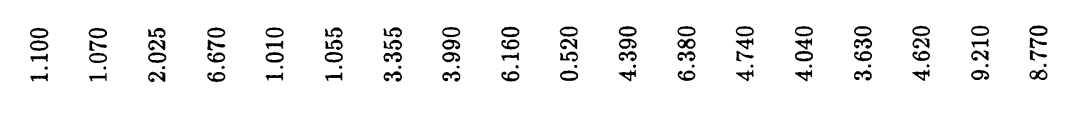 & 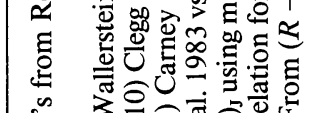 \\
\hline 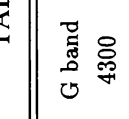 & 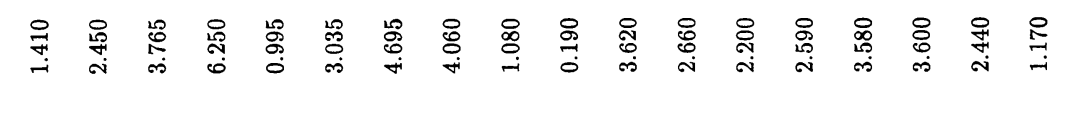 & \\
\hline 要 & 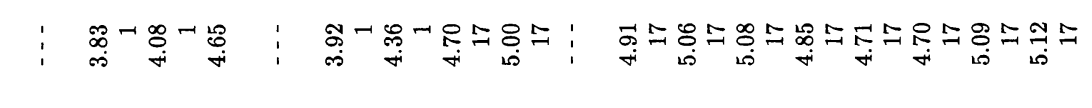 & \\
\hline 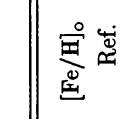 & 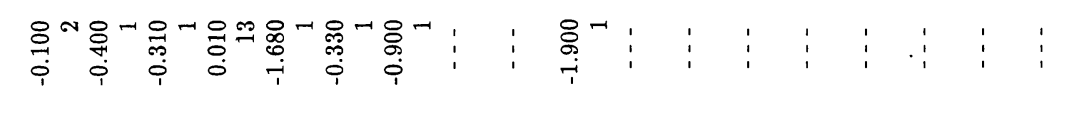 & \\
\hline $\bar{x}$ & 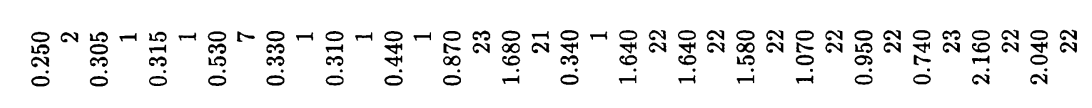 & 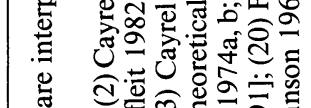 \\
\hline 旁蜜 & 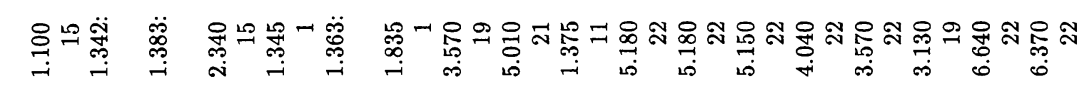 & \\
\hline 窇蜜 & 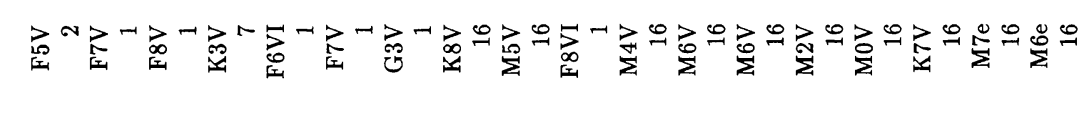 & 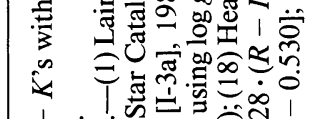 \\
\hline 急 & 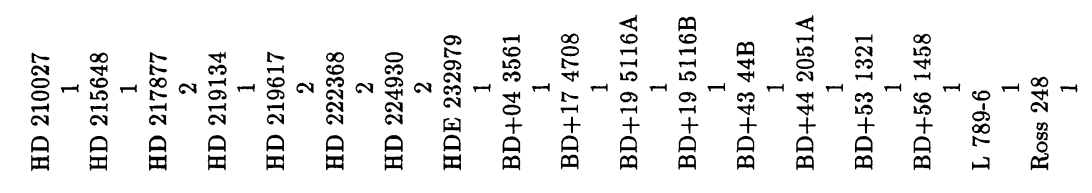 & 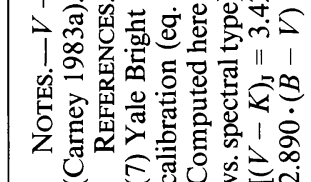 \\
\hline
\end{tabular}


TABLE 3

Cluster STARS ${ }^{\mathrm{a}}$

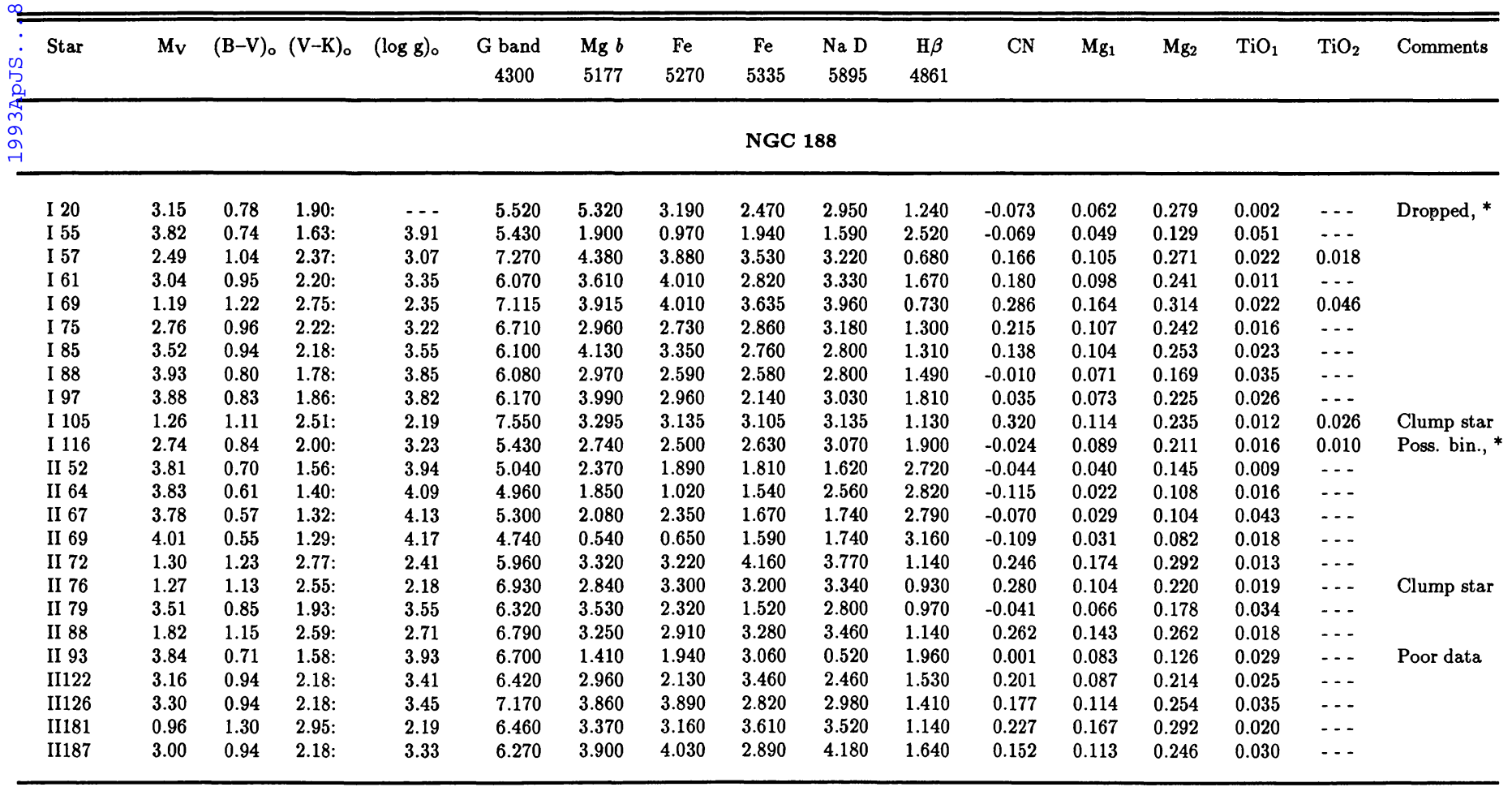

HYADES

\begin{tabular}{|c|c|c|c|c|c|c|c|c|c|c|c|c|c|c|c|}
\hline HR1346 & 0.20 & 0.99 & 2.14 & 2.77 & 6.260 & 2.570 & 3.070 & 3.140 & 2.550 & 1.820 & 0.237 & 0.058 & 0.164 & 0.017 & 0.010 \\
\hline HR1373 & 0.31 & 0.99 & 2.14 & 2.78 & 5.920 & 3.000 & 3.280 & 3.210 & 2.490 & 1.880 & 0.234 & 0.064 & 0.173 & 0.000 & 0.008 \\
\hline HR1411 & 0.38 & 0.95 & 2.09 & 2.78 & 5.710 & 2.580 & 3.150 & 2.460 & 2.290 & 1.750 & 0.188 & 0.046 & 0.163 & 0.015 & 0.010 \\
\hline
\end{tabular}

NGC 7789

\begin{tabular}{|c|c|c|c|c|c|c|c|c|c|c|c|c|c|c|c|c|}
\hline K 338 & -0.50 & 0.87 & 2.07: & $\ldots$ & 6.150 & 1.700 & 2.980 & 2.190 & 3.060 & 1.650 & 0.074 & 0.022 & 0.136 & 0.009 & 0.008 & Dropped, * \\
\hline K 415 & -1.63 & 1.59 & 3.88: & 1.06 & 7.030 & 4.550 & 4.070 & 4.040 & 5.200 & 0.370 & 0.117 & 0.222 & 0.429 & 0.137 & 0.313 & \\
\hline K 468 & -1.16 & 1.33 & 3.03: & 1.60 & 7.140 & 3.560 & 4.420 & 3.700 & 4.500 & 0.490 & 0.244 & 0.163 & 0.327 & 0.031 & 0.074 & \\
\hline К 489 & -1.05 & 1.15 & 2.60: & $\ldots$ & 5.960 & 3.410 & 4.190 & 3.440 & 4.140 & 1.190 & 0.170 & 0.145 & 0.295 & 0.018 & 0.057 & Dropped, * \\
\hline K 501 & -1.07 & 1.42 & 3.28: & 1.38 & 7.420 & 3.960 & 4.420 & 3.990 & 5.170 & 0.360 & 0.187 & 0.211 & 0.379 & 0.042 & 0.119 & \\
\hline K 575 & -0.18 & 1.15 & $2.60:$ & 1.98 & 6.760 & 3.080 & 3.050 & 2.890 & 3.320 & 1.250 & 0.251 & 0.092 & 0.228 & 0.018 & 0.035 & \\
\hline K 669 & -0.81 & 1.34 & 3.05: & 1.57 & 7.810 & 3.680 & 4.450 & 3.370 & 4.410 & 0.370 & 0.270 & 0.167 & 0.326 & 0.030 & 0.064 & \\
\hline K 676 & 0.56 & 0.90 & 2.12: & 2.32 & 6.890 & 2.150 & 3.320 & 2.450 & 2.560 & 1.060 & 0.099 & 0.050 & 0.148 & 0.013 & 0.010 & · \\
\hline K 859 & 0.41 & 1.08 & $2.46:$ & 2.27 & 6.360 & 2.820 & 3.380 & 3.300 & 3.290 & 1.300 & 0.121 & 0.086 & 0.213 & 0.025 & 0.031 & \\
\hline K 875 & 0.63 & 0.92 & 2.16: & 2.36 & 7.490 & 2.490 & 3.400 & 1.870 & 2.650 & 1.490 & 0.128 & 0.048 & 0.151 & 0.014 & 0.012 & \\
\hline K 897 & 0.61 & 0.92 & 2.16: & 2.35 & 6.080 & 2.000 & 2.770 & 2.390 & 2.530 & 1.830 & 0.123 & 0.049 & 0.153 & 0.020 & 0.019 & \\
\hline K 971 & -1.30 & 1.61 & 3.96: & 1.03 & 6.700 & 4.970 & 3.890 & 3.900 & 5.440 & 1.020 & 0.099 & 0.213 & 0.450 & 0.184 & 0.398 & \\
\hline
\end{tabular}

M67

\begin{tabular}{|c|c|c|c|c|c|c|c|c|c|c|c|c|c|c|c|c|}
\hline F 84 & 1.09 & 1.06 & 2.32 & 2.23 & 6.250 & 2.810 & 3.420 & 2.460 & 2.370 & 1.060 & 0.228 & 0.070 & 0.177 & 0.018 & -0.005 & Clump star \\
\hline F 94 & 3.33 & 0.50 & 1.21 & 4.07 & 3.420 & 1.570 & 1.770 & 1.310 & 1.790 & 3.460 & -0.075 & 0.000 & 0.079 & 0.000 & -0.008 & \\
\hline F 105 & 0.80 & 1.20 & 2.66 & 2.23 & 6.570 & 3.740 & 3.690 & 3.380 & 3.680 & 1.050 & 0.261 & 0.133 & 0.287 & 0.012 & 0.034 & \\
\hline F 108 & 0.22 & 1.32 & 2.98 & 1.83 & 6.320 & 3.740 & 3.770 & 3.730 & 3.900 & 0.980 & 0.248 & 0.186 & 0.330 & 0.030 & 0.059 & \\
\hline F 115 & 3.15 & 0.57 & 1.30 & 3.89 & 4.290 & 1.250 & 1.690 & 1.075 & 1.215 & 3.245 & -0.082 & 0.014 & 0.076 & 0.008 & -0.009 & \\
\hline F 117 & 3.11 & 0.71 & 1.86 & 3.79 & 4.175 & 2.195 & 2.095 & 2.045 & 1.835 & 2.250 & -0.048 & 0.050 & 0.160 & 0.018 & 0.013 & $\mathrm{SB}, *$ \\
\hline F 119 & 3.07 & 0.53 & $1.25:$ & 3.94 & 3.190 & 1.420 & 1.740 & 1.650 & 0.440 & 3.380 & -0.072 & 0.016 & 0.086 & 0.020 & 0.017 & $\mathrm{SB}$ \\
\hline F 125 & 4.36 & 0.52 & 1.23: & 4.34 & 3.360 & 1.890 & 1.010 & 0.920 & 0.330 & 3.250 & -0.081 & 0.008 & 0.082 & 0.020 & 0.011 & \\
\hline F 164 & 1.05 & 1.07 & 2.37 & 2.22 & 5.760 & 2.590 & 3.420 & 2.430 & 2.930 & 0.940 & 0.231 & 0.071 & 0.190 & 0.018 & -0.002 & Clump star \\
\hline F 170 & 0.19 & 1.30 & 2.92 & 1.83 & 6.190 & 3.700 & 3.890 & 3.370 & 4.180 & 0.820 & 0.260 & 0.167 & 0.302 & 0.018 & 0.050 & SB \\
\hline
\end{tabular}


TABLE 3-Continued

\begin{tabular}{|c|c|c|c|c|c|c|c|c|c|c|c|c|c|c|c|c|c|}
\hline $\begin{array}{l}100 \\
1 \\
1 \\
1 \\
1 \\
1 \\
1\end{array}$ & Star & $\mathrm{M}_{\mathbf{V}}$ & $(B-V)_{0}$ & $(\mathrm{~V}-\mathrm{K})_{\mathrm{o}}$ & $(\log g)_{\circ}$ & $\begin{array}{c}G \text { band } \\
4300\end{array}$ & $\begin{array}{l}\mathrm{Mg} b \\
5177\end{array}$ & $\begin{array}{c}\mathrm{Fe} \\
5270\end{array}$ & $\begin{array}{c}\mathrm{Fe} \\
5335\end{array}$ & $\begin{array}{r}\mathrm{Na} D \\
5895\end{array}$ & $\begin{array}{c}\mathrm{H} \beta \\
4861\end{array}$ & $\mathrm{CN}$ & $\mathrm{Mg}_{1}$ & $\mathrm{Mg}_{2}$ & $\mathrm{TiO}_{1}$ & $\mathrm{TiO}_{2}$ & Comments \\
\hline 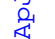 & F 175 & 4.22 & 0.54 & 1.27: & 4.34 & 3.630 & 1.070 & 1.690 & 0.720 & 0.850 & 3.780 & -0.088 & 0.009 & 0.081 & 0.025 & 0.006 & \\
\hline Im! & F 193 & 2.76 & 0.96 & 2.13 & 3.30 & 6.100 & 3.520 & 2.780 & 2.490 & 2.830 & 1.740 & 0.114 & 0.055 & 0.199 & 0.017 & 0.011 & \\
\hline InI & F 224 & 1.26 & 1.07 & 2.36 & 2.53 & 5.850 & 3.250 & 3.450 & 2.930 & 3.620 & 1.130 & 0.283 & 0.061 & 0.215 & 0.007 & 0.025 & SB, clump? * \\
\hline II] & F 231 & 2.00 & 0.99 & 2.22 & 2.95 & 6.550 & 3.190 & 2.870 & 3.030 & 3.160 & 1.420 & 0.176 & 0.072 & 0.205 & 0.007 & 0.009 & \\
\hline & I 17 & 2.90 & 0.93 & 2.14 & 3.37 & 6.375 & 3.780 & 2.915 & 2.570 & 2.465 & 1.625 & 0.116 & 0.069 & 0.206 & 0.013 & -0.001 & \\
\hline & II 22 & 3.43 & 0.87 & 2.00 & 3.65 & 6.800 & 3.320 & 2.900 & 2.470 & 2.390 & 1.480 & 0.047 & 0.071 & 0.197 & 0.019 & -0.007 & SB \\
\hline & IV 20 & 1.71 & 1.01 & 2.44 & 2.75 & 6.500 & 3.170 & 3.080 & 2.580 & 2.820 & 1.130 & 0.197 & 0.066 & 0.204 & 0.000 & 0.014 & $*$ \\
\hline & IV 68 & 3.45 & 0.81 & 1.94 & 3.73 & 5.630 & 2.430 & 2.210 & 1.860 & 1.790 & 1.620 & -0.009 & 0.048 & 0.164 & 0.007 & 0.000 & $*$ \\
\hline & IV 77 & 3.39 & 0.92 & 2.06 & 3.57 & 6.210 & 3.420 & 2.660 & 2.780 & 2.680 & 1.690 & 0.068 & 0.070 & 0.204 & 0.010 & 0.000 & \\
\hline & IV 81 & 3.29 & 0.76 & 1.73 & 3.76 & 5.550 & 2.470 & 2.340 & 2.270 & 2.230 & 2.390 & 0.005 & 0.032 & 0.156 & 0.017 & 0.001 & \\
\hline
\end{tabular}

M71

\begin{tabular}{|c|c|c|c|c|c|c|c|c|c|c|c|c|c|c|c|c|}
\hline 9 & -0.11 & 1.00 & 2.38: & 1.67 & 7.200 & 2.630 & 2.410 & 2.250 & 2.040 & 1.340 & 0.174 & 0.049 & 0.141 & 0.019 & 0.024 & \\
\hline 21 & -0.38 & 1.22 & 2.73 & 1.46 & 6.460 & 3.100 & 2.490 & 2.410 & 1.940 & 1.140 & 0.084 & 0.085 & 0.193 & 0.018 & 0.026 & \\
\hline 31 & 0.92 & 0.62 & 1.75: & $\cdots$ & 5.050 & 3.210 & 3.990 & 2.020 & 2.260 & 1.920 & -0.008 & 0.022 & 0.135 & 0.041 & 0.009 & Dropped, * \\
\hline 34 & 1.05 & 0.78 & 2.01: & 2.47 & 3.550 & 1.970 & 2.450 & 1.670 & 2.350 & 1.150 & -0.057 & 0.028 & 0.042 & 0.041 & 0.000 & \\
\hline 36 & -0.61 & 0.98 & 2.34: & $\cdots$ & 6.29 & 3.095 & 2.460 & 2.035 & 2.305 & 0.920 & 0.008 & C.112 & 0.221 & 0.024 & 0.046 & Dropped, * \\
\hline 37 & 0.76 & 1.06 & 2.49: & 2.18 & 6.370 & 2.530 & 2.240 & 1.680 & 2.050 & 0.820 & 0.137 & 0.065 & 0.158 & 0.025 & $\ldots$ & \\
\hline 39 & 1.12 & 0.83 & 2.09: & 2.43 & 7.560 & 2.010 & 2.880 & 0.200 & 2.080 & 0.620 & -0.028 & 0.013 & 0.090 & 0.038 & -0.005 & \\
\hline 41 & 1.01 & 0.76 & 1.97: & 2.48 & 5.670 & 2.660 & 2.320 & 1.350 & 1.140 & 1.680 & -0.058 & 0.011 & 0.081 & 0.036 & 0.011 & $*$ \\
\hline 53 & -0.43 & 1.34 & 3.06: & 1.42 & 6.000 & 3.460 & 3.180 & 2.270 & 2.390 & 0.770 & 0.225 & 0.132 & 0.254 & 0.026 & $\ldots$ & \\
\hline 59 & 1.23 & 1.03 & 2.43: & 2.44 & 7.120 & 2.240 & 2.620 & 1.440 & 2.320 & 1.170 & 0.000 & 0.066 & 0.162 & 0.048 & $\cdots$ & \\
\hline 63 & 0.13 & 0.99 & 2.36: & 1.82 & 6.680 & 1.280 & 2.430 & 1.350 & 1.790 & 1.330 & 0.157 & 0.038 & 0.116 & 0.007 & $\cdots$ & \\
\hline 64 & -0.30 & 1.26 & 2.88: & 1.51 & 6.910 & 3.480 & 3.040 & 2.130 & 3.020 & 0.450 & 0.084 & 0.141 & 0.263 & 0.039 & $\ldots$ & \\
\hline 65 & 0.80 & 1.04 & $2.45:$ & 2.20 & 6.110 & 2.620 & 2.670 & 1.640 & 2.550 & 1.030 & 0.186 & 0.071 & 0.155 & 0.035 & $\ldots$ & \\
\hline 66 & -0.39 & 1.13 & 2.62: & 1.47 & 6.975 & 3.050 & 2.700 & 2.595 & 2.460 & 0.630 & 0.144 & 0.132 & 0.248 & 0.020 & 0.032 & \\
\hline 71 & 0.14 & 1.17 & 2.69: & 1.81 & 6.880 & 2.780 & 2.530 & 1.980 & 1.230 & 0.430 & 0.039 & 0.102 & 0.207 & 0.016 & - . & \\
\hline 73 & 1.39 & 0.93 & 2.26: & 2.52 & 6.870 & 2.240 & 2.190 & 0.930 & 2.370 & 1.200 & 0.182 & 0.056 & 0.140 & 0.014 & 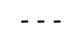 & \\
\hline 75 & 1.45 & 1.02 & 2.14 & 2.56 & 6.190 & 2.580 & 2.140 & 0.950 & 2.590 & 1.380 & 0.146 & 0.049 & 0.154 & 0.014 & $\ldots$ & \\
\hline 87 & 0.97 & 0.78 & 2.01: & 2.47 & 6.550 & 1.790 & 1.840 & 1.080 & 2.030 & 1.980 & -0.029 & 0.029 & 0.098 & 0.016 & 0.012 & \\
\hline 95 & -0.10 & 1.02 & 2.41: & 1.67 & 7.050 & 1.990 & 2.560 & 1.970 & 1.980 & 1.440 & 0.248 & 0.047 & 0.135 & 0.015 & 0.017 & \\
\hline 107 & 0.30 & 0.86 & 2.14: & 1.93 & 6.020 & 1.270 & 2.270 & 1.530 & 1.290 & 1.250 & -0.021 & 0.028 & 0.112 & 0.018 & $\ldots$ & \\
\hline 109 & 1.47 & 0.97 & 2.33: & 2.57 & 6.190 & 1.760 & 1.110 & 2.040 & 1.350 & 0.580 & 0.033 & 0.056 & 0.135 & 0.018 & $\ldots$ & \\
\hline 255 & 1.70 & 0.19 & 1.05: & $\cdots$ & 0.080 & 0.960 & 0.730 & -1.730 & 2.630 & 6.580 & -0.223 & -0.014 & 0.048 & 0.048 & 0.040 & Dropped, * \\
\hline A 2 & 1.13 & 0.93 & 2.19 & 2.37 & 6.995 & 2.615 & 2.030 & 1.560 & 2.065 & 1.495 & -0.024 & 0.060 & 0.150 & 0.018 & 0.016 & \\
\hline A 4 & -1.22 & 1.40 & 3.40 & 0.74 & 6.150 & 3.850 & 3.040 & 3.150 & 2.650 & 0.670 & - - & 0.205 & 0.344 & 0.046 & 0.116 & \\
\hline A 6 & -0.98 & 1.51 & 3.55 & $\cdots$ & 5.970 & 3.790 & 3.960 & 3.940 & 4.290 & 0.780 & $\ldots$ & 0.222 & 0.370 & 0.049 & 0.147 & Dropped, * \\
\hline A 7 & 0.05 & 1.18 & 2.53 & $\cdots$ & 6.445 & 3.345 & 3.705 & 3.150 & 3.245 & 1.370 & 0.189 & 0.114 & 0.256 & 0.014 & 0.037 & Dropped, * \\
\hline A 9 & -0.47 & 1.33 & 3.15 & 1.39 & 6.470 & 3.120 & 2.920 & 2.280 & 2.640 & 0.890 & - - & 0.144 & 0.267 & 0.017 & 0.058 & \\
\hline $\mathrm{C}$ & 1.09 & 0.89 & 2.16 & 2.39 & 6.700 & 2.470 & 1.585 & 1.520 & 1.810 & 0.875 & -0.022 & 0.056 & 0.140 & 0.016 & 0.017 & \\
\hline KC 22 & 0.53 & 1.10 & 2.56: & $\cdots$ & 6.870 & 4.080 & 3.240 & 2.650 & 2.830 & 1.110 & 0.177 & 0.119 & 0.269 & -0.008 & -- & Dropped, * \\
\hline $\mathrm{KC} 147$ & 1.60 & 0.87 & 2.16: & 2.64 & 6.870 & 2.310 & 2.360 & 1.880 & 1.130 & 1.540 & -0.064 & 0.044 & 0.138 & 0.009 & $\ldots$ & \\
\hline KC169 & 0.90 & 0.81 & 2.06: & 2.44 & 4.960 & 1.470 & 1.600 & 1.080 & 1.590 & 1.680 & 0.035 & 0.012 & 0.072 & 0.009 & $\ldots$ & \\
\hline KC263 & 1.65 & 0.88 & $2.17:$ & 2.66 & 6.570 & 3.340 & 2.330 & 1.780 & 0.900 & 1.200 & -0.064 & 0.028 & 0.144 & 0.012 & -- & \\
\hline S & -0.47 & 1.26 & 2.99 & 1.39 & 6.625 & 2.765 & 3.125 & 2.410 & 2.235 & 1.015 & 0.226 & 0.102 & 0.220 & 0.019 & 0.032 & \\
\hline $\mathrm{X}$ & 1.00 & 0.80 & 1.83 & 2.49 & 5.350 & 1.180 & 1.910 & 1.040 & 1.670 & 1.710 & 0.012 & 0.006 & 0.074 & 0.006 & 0.005 & \\
\hline $\mathrm{Z}$ & 1.31 & 0.35 & 1.31: & $\cdots$ & -0.510 & 2.160 & 2.710 & 0.920 & 1.080 & 5.330 & -0.106 & -0.012 & 0.034 & 0.029 & 0.039 & Dropped, * \\
\hline $\mathrm{Q}$ & 1.38 & 0.35 & $\ldots$ & $\cdots$ & 2.720 & 1.420 & 1.230 & 1.730 & 1.200 & 2.470 & -0.069 & 0.001 & 0.063 & 0.005 & 0.003 & Dropped, * \\
\hline
\end{tabular}

M3

\begin{tabular}{|c|c|c|c|c|c|c|c|c|c|c|c|c|c|c|c|}
\hline IV 25 & -1.35 & 1.12 & 2.82 & 1.21 & 6.170 & 0.700 & 1.770 & 1.560 & 1.080 & 1.250 & $\ldots$ & 0.031 & 0.072 & 0.012 & 0.011 \\
\hline III28 & -2.16 & 1.36 & 3.21 & 0.73 & 5.410 & 1.030 & 2.570 & 1.370 & 0.610 & 1.140 & $\ldots$ & 0.044 & 0.095 & 0.018 & 0.012 \\
\hline 98 & -0.94 & 1.03 & $2.60:$ & 1.44 & 5.870 & 0.760 & 1.570 & 1.230 & 1.140 & 1.120 & $\cdots$ & 0.026 & 0.074 & 0.017 & 0.005 \\
\hline
\end{tabular}


TABLE 3-Continued

\begin{tabular}{|c|c|c|c|c|c|c|c|c|c|c|c|c|c|c|c|c|}
\hline Star & $\mathbf{M}_{\mathbf{V}}$ & $(B-V)_{\circ}$ & $(\mathrm{V}-\mathrm{K})_{\mathrm{o}}$ & $(\log g)_{0}$ & $\begin{array}{c}G \text { band } \\
4300\end{array}$ & $\begin{array}{c}\mathrm{Mg} b \\
5177\end{array}$ & $\begin{array}{c}\mathrm{Fe} \\
5270\end{array}$ & $\begin{array}{c}\mathrm{Fe} \\
5335\end{array}$ & $\begin{array}{r}\mathrm{Na} \text { D } \\
5895\end{array}$ & $\begin{array}{c}\text { Н } \beta \\
4861\end{array}$ & $\mathrm{CN}$ & $\mathrm{Mg}_{1}$ & $\mathrm{Mg}_{2}$ & $\mathrm{TiO}_{1}$ & $\mathrm{TiO}_{2}$ & Comments \\
\hline & \multicolumn{16}{|c|}{ M5 } \\
\hline $\begin{array}{l}\text { III } 3 \\
\text { IV } 19 \\
\text { II } 51 \\
\text { IV } 86 \\
\text { IV } 59\end{array}$ & $\begin{array}{r}-2.01 \\
-1.74 \\
-0.34 \\
0.60 \\
-1.38\end{array}$ & $\begin{array}{l}1.45 \\
1.35 \\
0.94 \\
0.58 \\
1.27\end{array}$ & $\begin{array}{l}3.26 \\
3.18 \\
2.53 \\
1.64 \\
2.91\end{array}$ & $\begin{array}{l}0.66 \\
0.84 \\
1.69 \\
2.46 \\
0.85\end{array}$ & $\begin{array}{l}6.330 \\
5.830 \\
4.390 \\
1.330 \\
6.710\end{array}$ & $\begin{array}{l}2.160 \\
2.240 \\
1.200 \\
0.610 \\
1.440\end{array}$ & $\begin{array}{l}2.940 \\
2.230 \\
1.750 \\
0.290 \\
2.690\end{array}$ & $\begin{array}{l}2.300 \\
2.350 \\
0.720 \\
0.750 \\
1.830\end{array}$ & $\begin{array}{l}1.160 \\
1.380 \\
1.070 \\
1.060 \\
1.340\end{array}$ & $\begin{array}{l}0.960 \\
0.960 \\
1.110 \\
2.450 \\
1.520\end{array}$ & $\begin{array}{l}\cdots \\
\cdots \\
\cdots \\
\cdots\end{array}$ & $\begin{array}{l}0.073 \\
0.069 \\
0.028 \\
0.008 \\
0.056\end{array}$ & $\begin{array}{l}0.162 \\
0.131 \\
0.091 \\
0.030 \\
0.120\end{array}$ & $\begin{array}{r}0.019 \\
0.016 \\
0.016 \\
-0.004 \\
0.013\end{array}$ & $\begin{array}{r}0.029 \\
0.020 \\
-0.001 \\
-0.005 \\
0.015\end{array}$ & \\
\hline \multicolumn{17}{|c|}{ M10 } \\
\hline $\begin{array}{l}15 \\
18 \\
\text { II } 76 \\
\text { III } 85\end{array}$ & $\begin{array}{l}-2.30 \\
-1.54 \\
-0.81 \\
-1.29\end{array}$ & $\begin{array}{l}1.44 \\
1.24 \\
0.97 \\
1.13\end{array}$ & $\begin{array}{l}3.38: \\
3.10: \\
2.56: \\
2.80:\end{array}$ & $\begin{array}{l}0.57 \\
1.05 \\
1.47 \\
1.20\end{array}$ & $\begin{array}{l}4.490 \\
5.620 \\
4.620 \\
5.350\end{array}$ & $\begin{array}{l}1.660 \\
0.460 \\
0.950 \\
0.930\end{array}$ & $\begin{array}{l}2.320 \\
1.850 \\
1.370 \\
2.180\end{array}$ & $\begin{array}{l}2.060 \\
1.690 \\
0.830 \\
1.580\end{array}$ & $\begin{array}{l}1.770 \\
2.030 \\
1.260 \\
1.710\end{array}$ & $\begin{array}{l}0.670 \\
0.530 \\
1.410 \\
1.230\end{array}$ & $\begin{array}{l}\cdots \\
\cdots \\
\cdots \\
\cdots\end{array}$ & $\begin{array}{l}0.057 \\
0.029 \\
0.017 \\
0.023\end{array}$ & $\begin{array}{l}0.139 \\
0.064 \\
0.046 \\
0.075\end{array}$ & $\begin{array}{l}0.039 \\
0.021 \\
0.012 \\
0.008\end{array}$ & $\begin{array}{l}0.090 \\
0.025 \\
0.005 \\
0.018\end{array}$ & \\
\hline \multicolumn{17}{|c|}{ M13 } \\
\hline $\begin{array}{l}171 \\
786 \\
818\end{array}$ & $\begin{array}{l}-1.51 \\
-2.23 \\
-0.29\end{array}$ & $\begin{array}{l}1.01 \\
1.56 \\
0.66\end{array}$ & $\begin{array}{l}\text { 2.75: } \\
3.57: \\
1.98:\end{array}$ & $\begin{array}{l}1.07 \\
0.62 \\
1.89\end{array}$ & $\begin{array}{l}5.070 \\
4.460 \\
2.150\end{array}$ & $\begin{array}{l}0.930 \\
0.700 \\
1.270\end{array}$ & $\begin{array}{l}1.560 \\
2.090 \\
1.180\end{array}$ & $\begin{array}{l}1.080 \\
2.180 \\
0.410\end{array}$ & $\begin{array}{l}1.500 \\
1.900 \\
1.380\end{array}$ & $\begin{array}{l}1.480 \\
1.230 \\
1.760\end{array}$ & $\cdots$ & $\begin{array}{l}0.017 \\
0.047 \\
0.010\end{array}$ & $\begin{array}{l}0.052 \\
0.094 \\
0.046\end{array}$ & $\begin{array}{l}0.008 \\
0.015 \\
0.014\end{array}$ & $\begin{array}{l}0.017 \\
0.046 \\
0.017\end{array}$ & \\
\hline \multicolumn{17}{|c|}{ M92 } \\
\hline XII 8 & -1.84 & 1.04 & 2.63 & 1.00 & 1.980 & 0.710 & 0.310 & 0.670 & 1.250 & 1.210 & $\cdots$ & -0.006 & 0.024 & 0.003 & -0.011 & \\
\hline IX 12 & -0.48 & 0.52 & 1.60: & 1.93 & 0.340 & 0.700 & -0.700 & 0.330 & 0.450 & 2.210 & $\cdots$ & -0.003 & 0.014 & -0.006 & -0.011 & \\
\hline III13 & -2.57 & 1.31 & 3.03 & 0.58 & 3.810 & 0.620 & 1.430 & 1.260 & 1.180 & 0.950 & $\cdots$ & 0.009 & 0.043 & 0.009 & -0.016 & \\
\hline IV114 & -0.77 & 0.85 & 2.35 & 1.58 & 2.130 & 0.520 & 0.100 & 0.730 & 1.040 & 1.060 & $\cdots$ & 0.006 & 0.023 & 0.010 & -0.001 & \\
\hline
\end{tabular}

${ }^{a}$ All colors and magnitudes are corrected for reddening. $V-K$ values with colons have been interpolated from $B-V$. Distance moduli are raw, including absorption. Sources of photometry and moduli are given in Table 4.

Star numbers given simply as numerals are from Osborn 1973.

NOTES ON SOURCES OF PROPER MOTIONS:

NGC 188: Upgren et al. 1972. All stars have probabilities of 0.89 or higher.

NGC 7789: McNamara \& Solomon 1981. All stars except as noted have probabilities of 0.87 or higher.

M71: Cudworth 1985. All stars except as noted have probabilities of 0.86 or higher.

M67: Sanders 1977. All stars have probabilities of 0.90 or higher.

NOTES ON INDIVIDUAL STARS:

NGC 188.- I20: Off cluster HR locus. Probable field dwarf based on $\mathrm{Mg}_{2}$. Dropped. I1 16: Possible binary based on position in CM digram, but agreement in indices satisfactory. Retained.

NGC 7789.-K338: Lies above giant branch. Janes (1977) suggests it may be a binary with a turnoff star, but this is ruled out by low H $\beta$. Dropped. K489: Proper motion probability 0.84 . Lies above giant branch. Dropped.

M67: Spectroscopic binaries and radial velocities from Mathieu \& Latham 1986. F117: Spectroscopic binary. Indices suggest hotter $T$, consistent with $B-V$. Observed $V-K$ may be too cool. F224: Spectroscopic binary. Could be clump star, but $\mathrm{Mg}_{2}$ and $\mathrm{MgH}$ both suggest higher gravity. We have assumed equal brightness components and placed on giant branch accordingly. IV 20: Radial velocity member, but proper motion probability is only 0.64 . Retained. Indices suggest hotter $T$, consistent with $B-V$. Observed $V-K$ may be too cool. IV 68: Indices suggest hotter temperature. Observed $V-K$ may be too cool.

M71: 31: Proper motion probability 0.00. Dropped. 36: Off giant branch. Indices discrepant. Proper motion probability 0.64. Dropped. 41: Proper motion probability 0.72 . Retained. 255: Proper motion probability 0.51 . Dropped. A 6: Proper motion probability 0.10 . Dropped. A 7: Proper motion probability 0.00 . Dropped. KC 22: Metal-rich field giant. Dropped Z: Proper motion probability 0.00. Dropped. Q: No proper motion available. Dropped. 


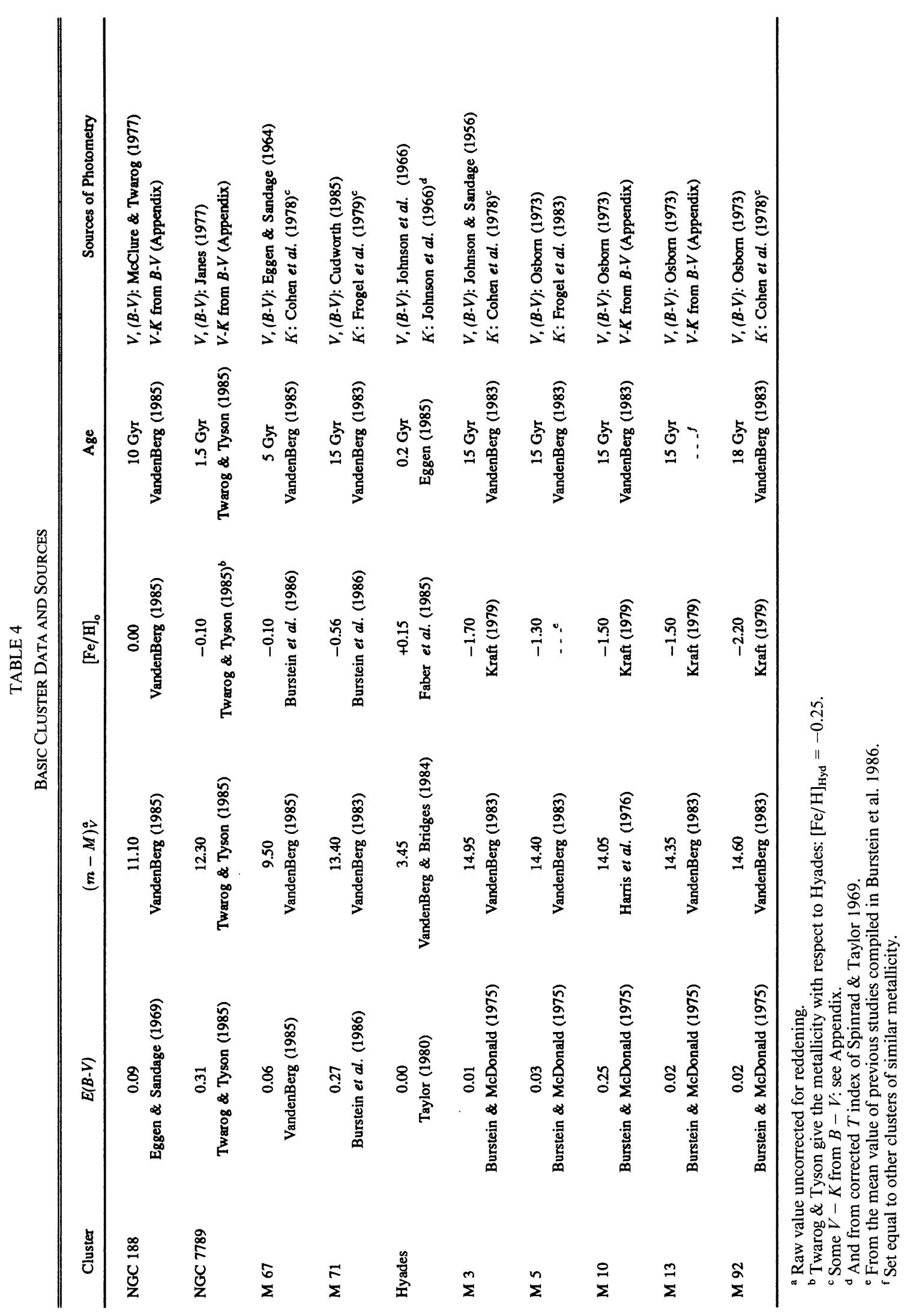


OLD STELLAR POPULATIONS. IV.

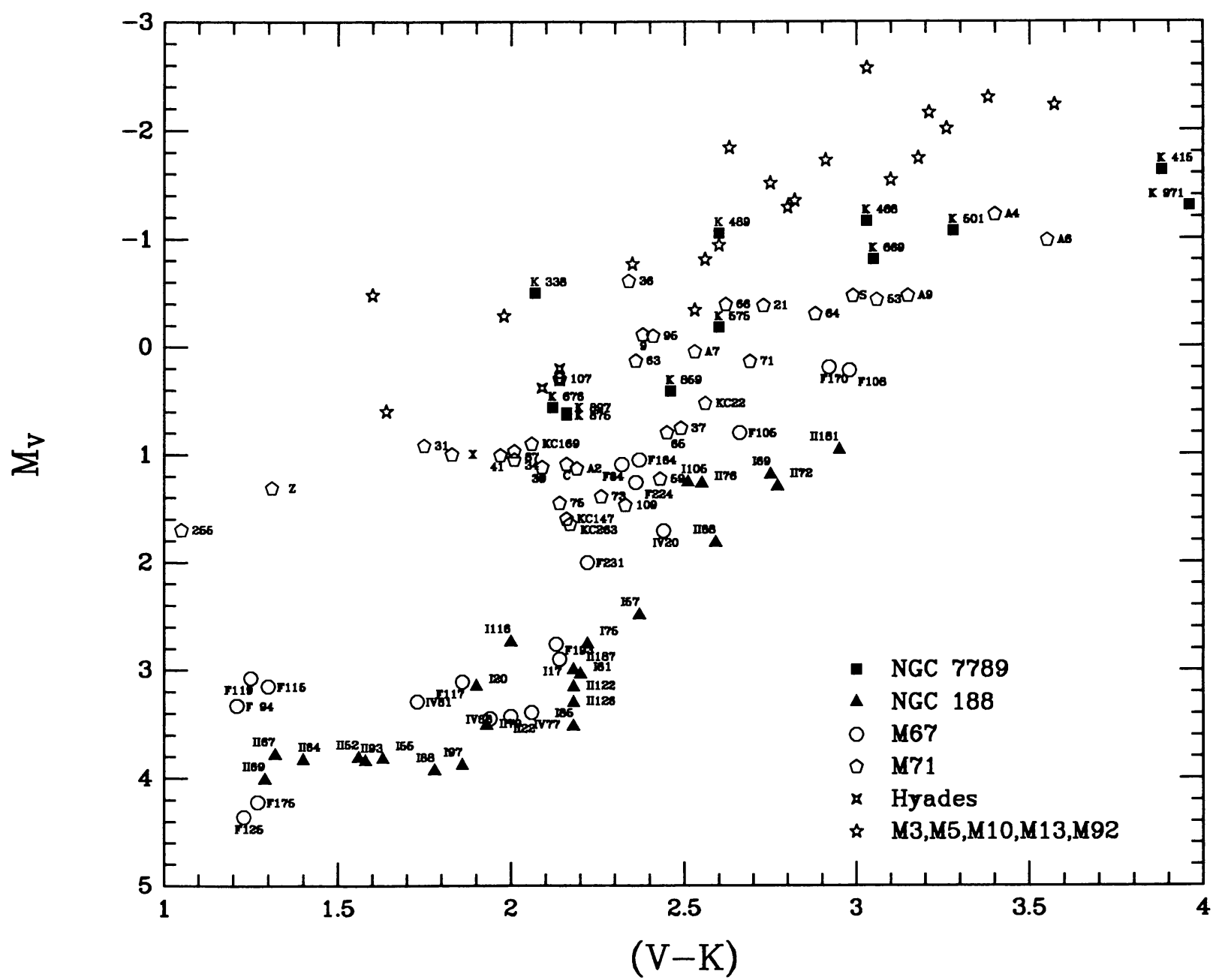

FIG. 1.-Composite, dereddened H-R diagram for cluster stars, including nonmembers. The colors and absolute magnitudes correspond to the reddenings and distance moduli in Table 4.

tions are less certain at metallicities much higher and lower than this value.

The second limitation reflects the fact that the local diskhalo abundance ratio trajectories, $[\mathrm{X} / \mathrm{Fe}]$ versus $[\mathrm{Fe} / \mathrm{H}]$, are also built into the fitting formulae. As these trajectories might not be obeyed in other stellar systems, the fitting functions should be applied to them experimentally and with caution. Discrepancies have already been noted for giant elliptical galaxies (Worthey, Faber, \& Gonzalez 1992).

For ease of understanding and display, it is desirable that the fitting functions be separable in both gravity and metallicity. To achieve this, we found it convenient to adopt two generic forms:

$$
I(V-K, g, Z) \equiv p(V-K, g)+q(V-K, Z)
$$

and

$$
\begin{aligned}
& I(V-K, g, Z) \\
& \quad \equiv \exp [p(V-K, g)+q(V-K, Z)]+\text { const } .
\end{aligned}
$$

Here $I$ is any index, $g$ is gravity $(\log g)$, and $Z$ is metallicity $([\mathrm{Fe} / \mathrm{H}])$. The functions $p$ and $q$ are assumed to be polynomials in $V-K, g$, and $Z$, with terms as described below. The metallicity function $q$ is defined to be zero for solar metallicity. Forms (1) and (2) were tried for each index, and the better fit was adopted. The constant term in equation (2) is a small negative offset introduced to remove negative values from the exponential fit and was adjusted to give the best fit. It generally optimizes at the smallest possible absolute value.

The function $q$ is a correction term for nonsolar abundance (additive in the case of eq. [1], multiplicative for eq. [2]). By adding (or multiplying) the correction in inversely, we can correct the observed index of any star to what it would be for solar metallicity. The expressions

$$
I^{g}(V-K, g) \equiv I-q(V-K, Z)
$$

and

$$
I^{g}(V-K, g) \equiv(I-\text { const. }) / \exp [q(V-K, Z)]
$$



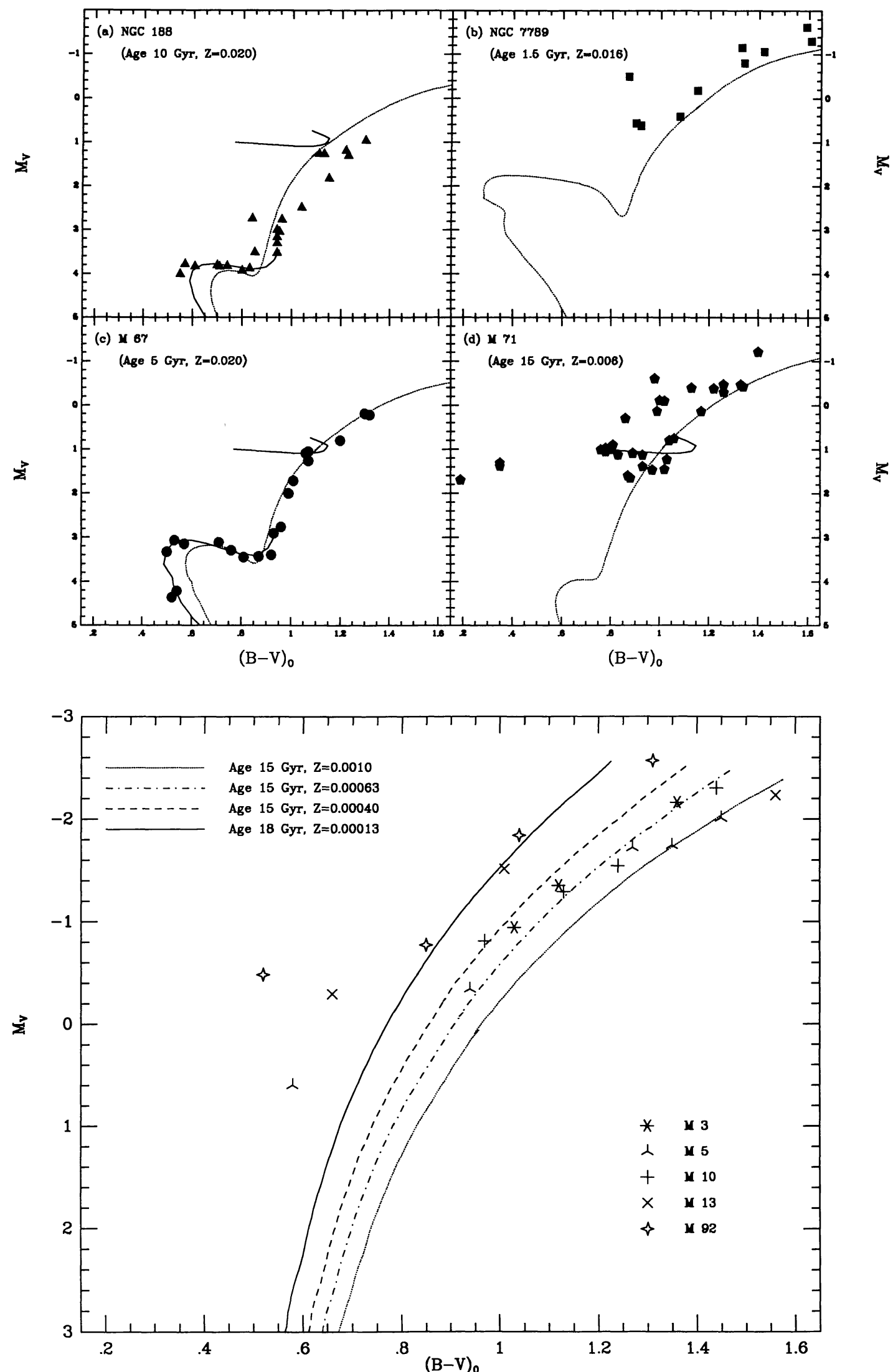

FIG. 2.-H-R diagrams and isochrones for individual clusters. $(a)-(d)$ Metal-rich clusters. Turnoff and horizontal-branch isochrones are from VandenBerg (1985) (solid lines); giant-branch isochrones are taken from Green et al. (1987; Yale) (whole isochrone shown as dotted lines). The ages and metallicities shown are those used to interpolate among the Yale tracks. The metallicities differ slightly from those in Table 4 because $Z_{\odot}$ for Yale is 0.020 rather than 0.0169 as used by VandenBerg. $(e)$ Metal-poor globular cluster isochrones as interpolated from the Yale tracks. The ages and metallicities shown correspond to the values in Table 4 (with $Z_{\odot} \equiv 0.020$ ). The isochrones are, from the top (solid line): M92, M3, M10 and M13, and M5. In all Yale interpolations, $Y=0.25$. Nonmembers (see Table 3 ) are not shown. 


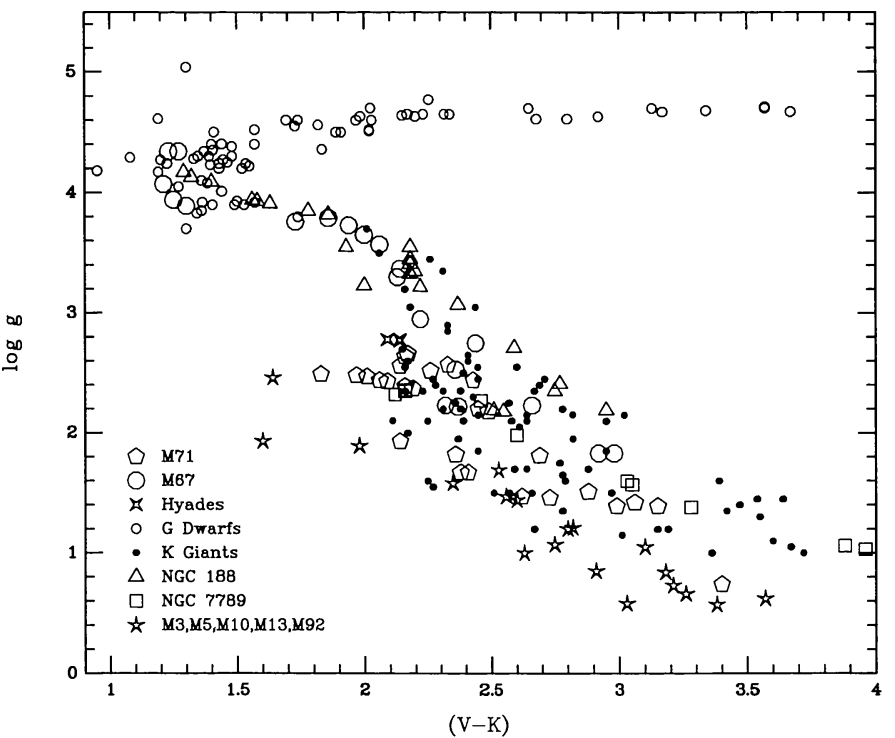

FIG. 3.- $(V-K)$, gravity diagram for all stars

are such corrected indices (the superscript $g$ signals that the effect of gravity is still present). If the fitting functions are correct, these observed corrected indices should match the gravity functions $p(V-K, g)$ and $\exp [p(V-K, g)]$, which show how the indices behave for solar metallicity stars. Analogously, the metallicity deviations

$$
I^{Z}(V-K, Z) \equiv I-p(V-K, g)
$$

and

$$
I^{Z}(V-K, Z) \equiv(I-\text { const. }) / \exp [p(V-K, g)]
$$

express the amount by which a star's index departs from nominal owing to nonsolar abundance (note the superscript $Z$ here). These observed quantities are to be compared with and should match well the metallicity functions $q(V-K, Z)$ and $\exp [q(V-K, Z)]$. We show many such comparisons of $I^{g}$ and $I^{Z}$ versus $p$ and $q$ below.

To achieve separability, we have excluded cross terms in $g$ and $Z$. The metallicity function $q$ is therefore constrained to be independent of gravity and thus must be the same for dwarfs and giants. We have found that adequate fits can be achieved under this assumption for all but two indices ( $\mathrm{CN}$ and $\mathrm{G})$. For these two, dwarfs and giants have had to be treated separately. ${ }^{7}$

After experimentation, the functions $p$ were taken to be polynomials with terms up to second order in $g$ and $V-K$, plus extra terms in $(V-K)^{3}, g(V-K)$, and a constant. The functions $q$ collect only terms involving the metallicity and contain terms in $Z, Z^{2}$, and $Z(V-K)$. The coefficients of the $p$ s and $q$ s are found using least squares with equal weights for all stars. For each index, we began with the smallest number of terms and increased it as necessary until systematic behavior in the

\footnotetext{
${ }^{7}$ But even in these cases, the problems are due to composition variations rather than surface gravity effects, and cross terms would not help. See discussion of these indices below.
}

residuals was acceptably reduced. The linear and exponential fits were tested separately, and the better fit was adopted.

Not all the fits are perfect. In some cases, higher-order terms might decrease the remaining residuals slightly, but we resisted adding them as the functions are already rather complicated, and the significance of the extra terms is doubtful. In a couple of cases, the problems are inherent in the data themselves, in the sense that data samples from the field and the various clusters seem to be mutually inconsistent. Discrepant cases and the magnitude of the remaining residuals are discussed further below.

\section{FITTING FUNCTIONS FOR THE INDIVIDUAL INDICES}

The basic input atmospheric parameters for all stars $(V-K$, $\log g$, and $\left.[\mathrm{Fe} / \mathrm{H}]_{0}\right)$ are summarized in Table 5. Values of input $[\mathrm{Fe} / \mathrm{H}]_{0}$ for a few field $\mathrm{K}$ giants have been revised from Paper II as described in the notes. Input gravities for field $\mathrm{K}$ giants were derived as explained in the next section.

\section{1. $\mathrm{Mg}_{2}$}

In order to make use of the field $\mathrm{K}$ giant sample, gravities must be provided for these stars. This was accomplished by starting with the index $\mathrm{Mg}_{2}$, which is most sensitive to gravity. $\mathrm{Mg}_{2}$ contains both the $\mathrm{Mg} b$ lines and $\mathrm{MgH}$ absorption. The strong gravity dependence of $\mathrm{Mg}_{2}$ is shown in Figure $4 a$, which shows the raw data versus $V-K$. At fixed $V-K$, the dwarf sequence clearly lies above the giants to the upper left, while the cluster sequences in M67 and NGC 188 gradually make the transition from the main-sequence locus through the subgiant region to the giant locus.

Using the exponential form in equation (2) (with constant term $=0$ ), we started by fitting the cluster stars and main-sequence dwarfs, whose gravities are known a priori. Dwarfs were limited to $V-K<2.50 \mathrm{mag}$, as no simple function is capable of matching the turnover in dwarf $\mathrm{Mg}_{2}$ strength cooler than this. With preliminary expressions for $p$ and $q$, we then plotted all stars (including the field $\mathrm{K}$ giants) in a diagram like Figure $4 c$, which shows the corrected index $\operatorname{Mg}_{2}^{g} \equiv$ $I / \exp [q(V-K, Z)]$ as defined by equation (4). Recall that this index shows the indices of all stars as they would appear at solar metallicity. The trends in Figure $4 c$ thus reflect the effects of temperature and gravity alone, as though all stars had solar abundance.

The light lines in Figure $4 c$ are the isogravity contours of the fitting function $p$. For each field $\mathrm{K}$ giant, we read off its gravity by interpolating between the contours. With these preliminary gravities, we then repeated the fit for $p$ and $q$, using now all stars. The large range in metallicities provided by the field $\mathrm{K}$ giants now provided better leverage on the metallicity dependence, and the new metallicity function was much improved. Updated gravities for the field $\mathrm{K}$ giants were derived once again, and the whole process was repeated. It converged well in two steps. The final derived gravities for the $\mathrm{K}$ giants are given in Table 5 .

It is important to note that, because the gravities of the field $\mathrm{K}$ giants have been force-fitted to $\mathrm{Mg}_{2}$, these stars yield identically zero residuals in the final fit for $\mathrm{Mg}_{2}$ versus gravity and metallicity. Furthermore, because $\mathrm{Mg}_{2}$ is closely correlated with $\mathrm{Mg}_{1}$, the residuals for the field $\mathrm{K}$ giants in this fit are 
TABLE 5

INPUT AND OUTPUT METALlicities AND GRAVITIES FOR All STARS USED IN SOlUTIONS

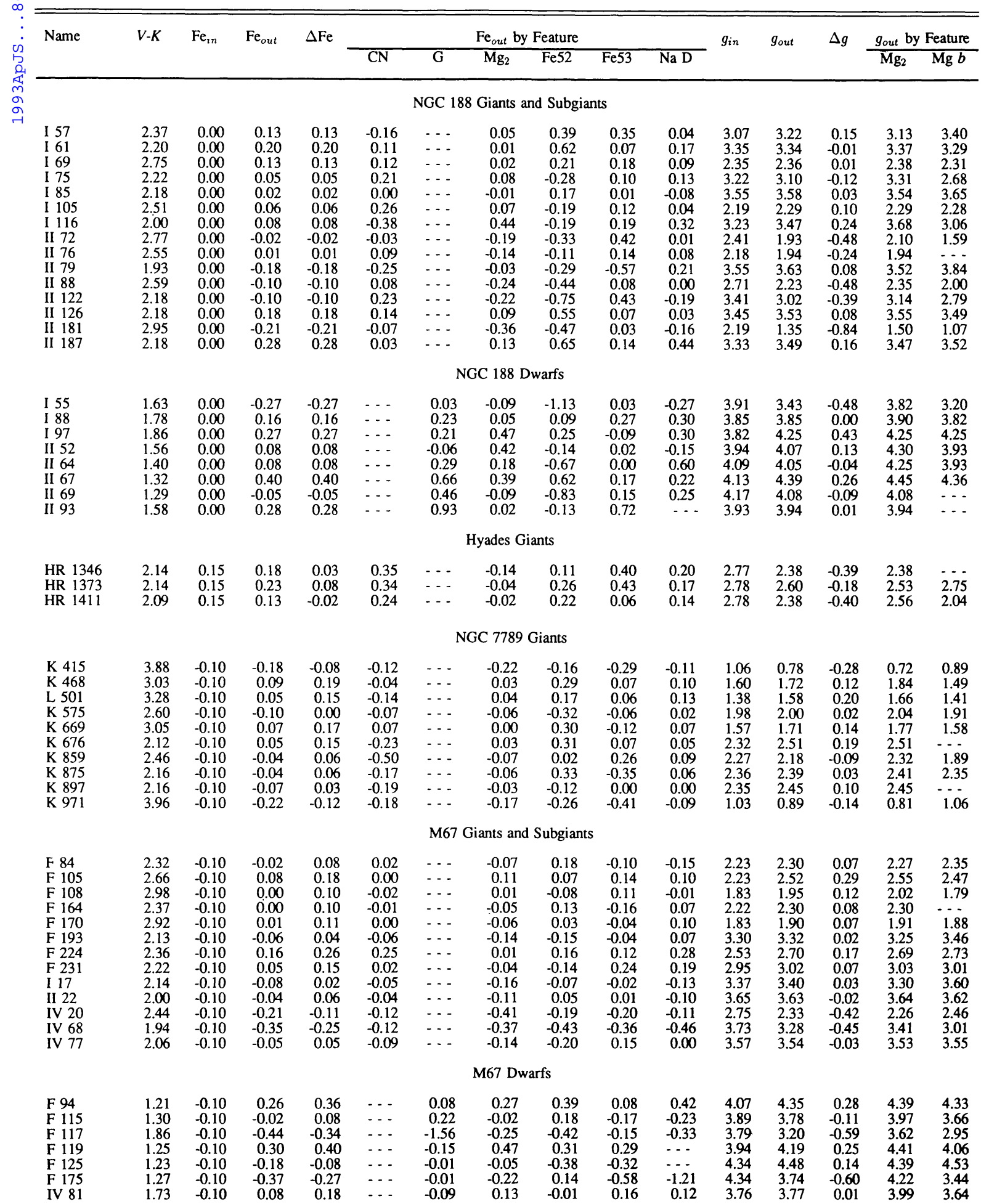

\begin{tabular}{|c|c|c|c|c|c|c|c|c|c|c|c|c|c|c|c|}
\hline \multicolumn{16}{|c|}{ M71 Giants } \\
\hline $\begin{array}{l}9 \\
21\end{array}$ & $\begin{array}{l}2.38 \\
2.73\end{array}$ & $\begin{array}{l}-0.56 \\
-0.566\end{array}$ & $\begin{array}{l}-0.41 \\
-0.66\end{array}$ & $\begin{array}{r}0.15 \\
-0.10\end{array}$ & $\begin{array}{l}-0.32 \\
-0.81\end{array}$ & $\cdots$ & $\begin{array}{r}-0.31 \\
-0.25\end{array}$ & -0.65 & -0.25 & -0.50 & 1.67 & 2.30 & & 2.14 & \\
\hline $\begin{array}{l}21 \\
34\end{array}$ & 2.01 & $\begin{array}{l}-0.56 \\
-0.56\end{array}$ & $\begin{array}{l}-0.66 \\
-0.58\end{array}$ & $\begin{array}{l}-0.10 \\
-0.02\end{array}$ & $\begin{array}{l}-0.81 \\
-0.63\end{array}$ & $\cdots$ & -0 & $\begin{array}{l}-0.86 \\
-010\end{array}$ & -0.44 & -0.82 & 1.46 & 2.04 & 0.58 & 1.92 & \\
\hline 37 & 2.49 & $\begin{array}{l}-0.56 \\
-0.56\end{array}$ & $\begin{array}{l}-0.66 \\
-0.66\end{array}$ & $\begin{array}{l}-0.02 \\
-0.10\end{array}$ & $\begin{array}{l}-0.63 \\
-0.47\end{array}$ & $\cdots$ & $\begin{array}{l}-1.7 \\
-0.6\end{array}$ & $\begin{array}{l}-0.1 \\
-0.8 \\
-0.8\end{array}$ & $\begin{array}{l}-0.35 \\
-0.83\end{array}$ & 0.0 & 2.47 & 2.83 & 0.36 & & \\
\hline 39 & 2.09 & -0.56 & -0.42 & 0.14 & -0.68 & $\ldots$ & -0.84 & 0.0 & & $\begin{array}{l}-0.56 \\
-0.20\end{array}$ & $\begin{array}{l}2.18 \\
2.43\end{array}$ & & -0.03 & & \\
\hline & 1.97 & -0.56 & -0.69 & -0.13 & -0.5 & $\ldots$ & -0.78 & -0.25 & -0.56 & $\begin{array}{l}-0.20 \\
-1.30\end{array}$ & 2.48 & $\begin{array}{l}2.15 \\
2.58\end{array}$ & $\begin{array}{r}-0.28 \\
0.10\end{array}$ & 1.90 & \\
\hline 53 & 3.06 & -0.56 & -0.51 & 0.05 & -0.13 & $\cdots$ & -0.38 & -0.52 & -0.82 & -0.68 & & & & & \\
\hline 59 & 2.43 & -0.56 & -0.68 & -0.12 & -0.9 & $\cdots$ & -0.59 & -0.50 & -1.0 & -0.3 & 2.44 & 2.2 & -0.20 & 2.39 & \\
\hline 63 & 2.36 & $\begin{array}{l}-0.56 \\
-0.56\end{array}$ & $\begin{array}{l}-0.65 \\
-0.48\end{array}$ & -0.09 & -0.35 & $\cdots$ & -0.67 & -0.60 & -0.95 & -0.68 & 1.82 & 1.58 & -0.24 & 1.58 & \\
\hline & & -0.56 & -0.48 & 0.08 & -0.77 & & -0.08 & -0.53 & & & & 2.39 & 0.88 & 2.44 & \\
\hline
\end{tabular}


TABLE 5-Continued

\begin{tabular}{|c|c|c|c|c|c|c|c|c|c|c|c|c|c|c|c|}
\hline \multirow[t]{2}{*}{ Namc } & \multirow[t]{2}{*}{$V \cdot K$} & \multirow[t]{2}{*}{$\mathrm{Fc}_{t \prime}$} & \multirow[t]{2}{*}{$\mathrm{Fc}_{,, u t}$} & \multirow[t]{2}{*}{$\Delta \mathrm{Fc}$} & \multicolumn{6}{|c|}{$\mathrm{Fc}_{, u t}$ by Featurc } & \multirow[t]{2}{*}{$y_{t \prime \prime}$} & \multirow[t]{2}{*}{ y.ut } & \multirow[t]{2}{*}{$\Delta g$} & \multicolumn{2}{|c|}{$g_{\text {out }}$ by Fcaturc } \\
\hline & & & & & $\overline{\mathrm{CN}}$ & $\bar{G}$ & $\mathrm{Mg}_{2}$ & Fc52 & Fe53 & $\mathrm{NaD}$ & & & & $\mathrm{Mg}_{2}$ & $\overline{M g} b$ \\
\hline & \multicolumn{15}{|c|}{ M 71 Giants (continucd) } \\
\hline 65 & 2.45 & -0.56 & -0.47 & 0.09 & -0.25 & $\cdots$ & -0.57 & -0.48 & -0.82 & -0.21 & 2.20 & 2.27 & 0.07 & 2.18 & 2.45 \\
\hline 66 & 2.62 & -0.56 & -0.29 & 0.27 & -0.57 & & 0.37 & -0.63 & .0 .23 & -0.39 & 1.47 & 2.81 & & & 2.47 \\
\hline 71 & 2.69 & -0.56 & -0.93 & -0.37 & -0.95 & & -0.32 & -0.78 & -0.74 & -1.87 & 1.81 & 2.20 & 0.39 & 2.27 & 2.07 \\
\hline 73 & 2.26 & -0.56 & -0.58 & -0.02 & -0.06 & $-\cdots$ & -0.54 & -0.68 & -1.44 & -0.17 & 2.52 & 2.53 & 0.01 & 2.56 & 2.48 \\
\hline 75 & 2.14 & -0.56 & -0.38 & 0.18 & -0.04 & $\cdots$ & -0.11 & -0.60 & -1.21 & 0.04 & 2.56 & 3.18 & 0.62 & 3.21 & 3.12 \\
\hline 87 & 2.01 & -0.56 & -0.56 & 0.00 & -0.52 & & -0.55 & -0.69 & -0.86 & -0.17 & 2.47 & 2.5 & 0.03 & 2.48 & 2.53 \\
\hline 95 & 2.41 & -0.56 & -0.42 & 0.14 & -0.04 & & -0.45 & -0.5 & -0.48 & -0.56 & 1.6 & 1.8 & 0.2 & 1.89 & \\
\hline 107 & 2.14 & -0.56 & -0.67 & -0.11 & -0.80 & & -0.31 & -0.51 & -0.56 & -1.19 & 1.93 & 2.3 & 0. & 2.36 & $\cdots$ \\
\hline 109 & 2.33 & -0.56 & -0.99 & -0.43 & -0.73 & & -0.76 & -1.78 & -0.43 & -1.28 & 2.57 & 2.2 & -0.37 & 2. & $\cdots$ \\
\hline A 2 & 2.19 & -0.56 & -0.53 & 0.03 & -0.82 & & -0.16 & -0.74 & -0.62 & $\begin{array}{l}-0.30 \\
\end{array}$ & 2.37 & 3.0 & 0.64 & 2.99 & 3.04 \\
\hline A 4 & 3.40 & -0.56 & -0.49 & 0.07 & $\cdots$ & - & -0.04 & -0.7 & -0.46 & -0.70 & 0.74 & 1.5 & 1. & 2.02 & 1.67 \\
\hline A 9 & 3.15 & -0.56 & -0.66 & -0.10 & $\ldots$ & & -0.41 & -0.74 & -0.89 & -0.58 & 1.39 & 1.66 & 0.27 & 1.75 & 1.49 \\
\hline & 2.16 & .0 .56 & -0.64 & -0.08 & -0.77 & & -0.22 & -1.11 & -0.62 & -0.48 & 2.39 & 2.93 & 0.54 & 2.91 & 2.98 \\
\hline KC 147 & 2.16 & -0.56 & -0.75 & -0.19 & -0.90 & & -0.42 & -0.44 & -0.37 & -1.60 & 2.64 & 2.8 & 0.2 & 2.87 & 2.83 \\
\hline KC 169 & 2.06 & -0.56 & -0.80 & -0.24 & -0.37 & $\cdots$ & -1.12 & -0.97 & -0.92 & -0.62 & 2.44 & 1.2 & -1 . & 1.25 & \\
\hline KC 263 & 2.17 & -0.56 & -0.56 & 0.00 & -0.92 & & -0.38 & -0.48 & -0.46 & & 2.66 & 3.15 & 0.49 & 2.94 & 3.58 \\
\hline$S$ & 2.99 & -0.56 & -0.52 & 0.04 & -0.16 & & -0.50 & -0.53 & -0.66 & -0.74 & 1.39 & 1.47 & 0.08 & 1.52 & 1.37 \\
\hline
\end{tabular}

M3 Giants

$\begin{array}{llllllllllllllll}\text { IV } 25 & 2.82 & -1.70 & -1.51 & 0.19 & \ldots & \ldots & -1.75 & -1.55 & -1.22 & \ldots & 1.21 & 1.04 & -0.17 & 1.04 & \ldots \\ \text { III 28 } & 3.21 & -1.70 & -1.56 & 0.14 & \ldots & \ldots . & -1.67 & -1.09 & -1.92 & -\ldots & 0.73 & 0.86 & 0.13 & 0.86 & \ldots \\ 98 & 2.60 & -1.70 & -1.49 & 0.21 & -\ldots & -\ldots & -1.52 & -1.58 & -1.36 & -\ldots & 1.44 & 1.95 & 0.51 & 1.95 & \ldots\end{array}$

M5 Giants

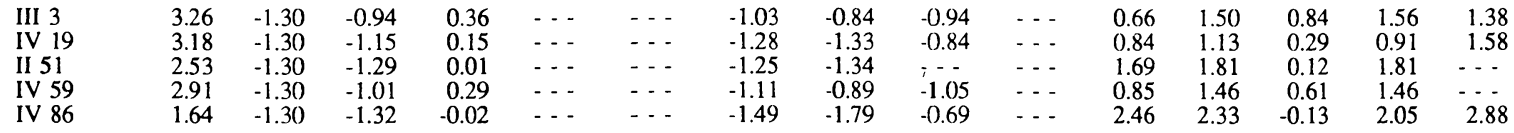

M10 Giants

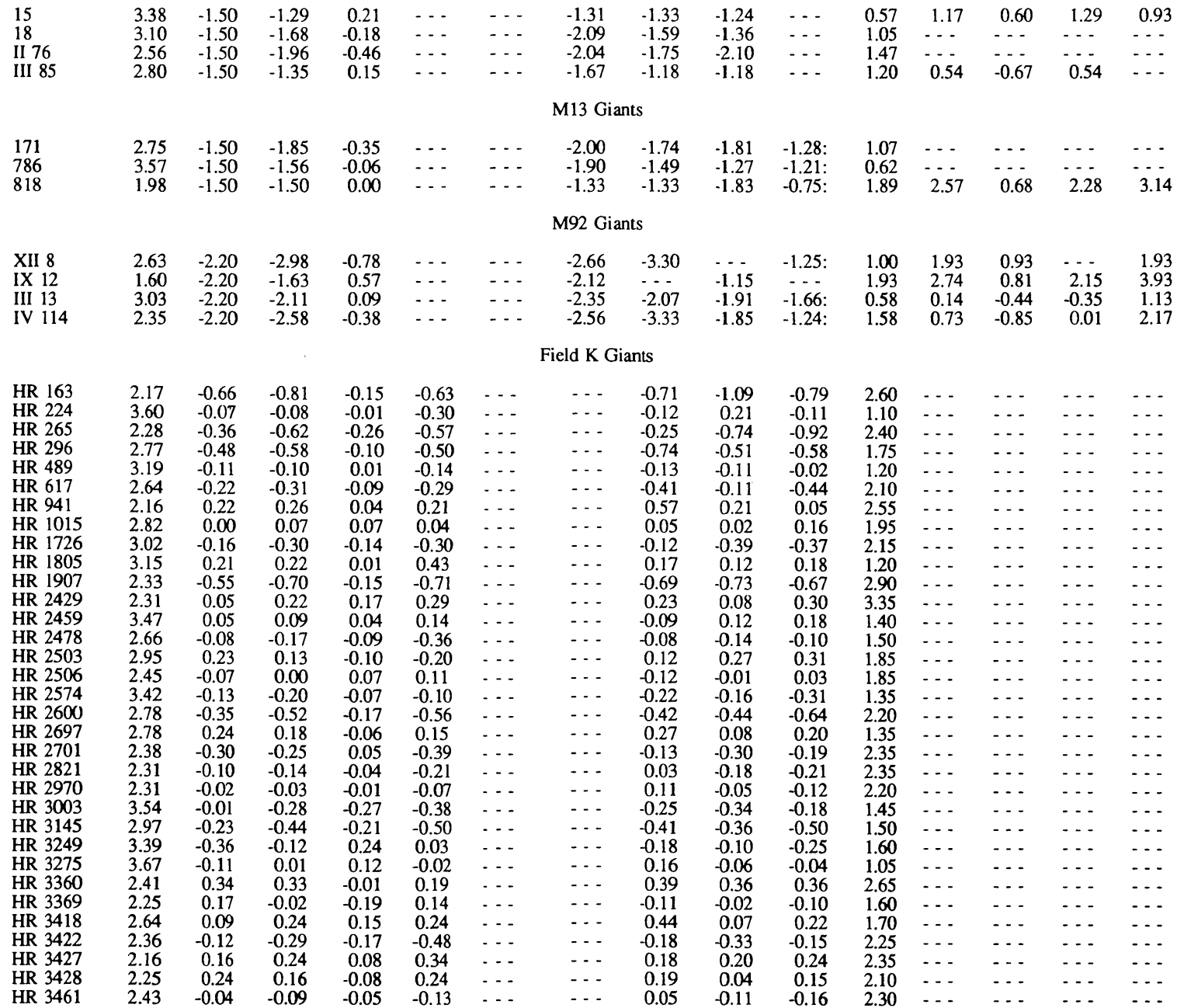


TABLE 5-Continued

\begin{tabular}{|c|c|c|c|c|c|c|c|c|c|c|c|c|c|c|c|}
\hline \multirow[t]{2}{*}{ Name } & \multirow[t]{2}{*}{$V-K$} & \multirow[t]{2}{*}{$\mathrm{Fe}_{\prime \prime \prime}$} & \multirow[t]{2}{*}{$\mathrm{Fc}_{\text {, } w t}$} & \multirow[t]{2}{*}{$\Delta \mathrm{Fe}$} & \multicolumn{6}{|c|}{$\mathrm{Fe}_{, \prime, t}$ by Feature } & \multirow[t]{2}{*}{$g_{\imath n}$} & \multirow[t]{2}{*}{$g_{\text {cout }}$} & \multirow[t]{2}{*}{$\Delta y$} & \multicolumn{2}{|c|}{$g_{\text {out }}$ by Featur } \\
\hline & & & & & $\mathrm{CN}$ & $\bar{G}$ & $\mathrm{Mg}_{2}$ & $\mathrm{Fe} 52$ & Fe53 & $\mathrm{NaD}$ & & & & $\mathrm{Mg}_{2}$ & $\mathrm{Mg} b$ \\
\hline & & & & & & & & & & & & & & & \\
\hline \multicolumn{16}{|c|}{ Ficld K Giants (continued) } \\
\hline HR 3845 & 3.01 & -0.05 & -0.03 & 0.02 & -0.08 & ... & ... & 0.05 & 0.12 & -0.22 & 1.15 & $\cdots$ & - - & ... & \\
\hline HR 3905 & 2.64 & 0.46 & 0.41 & -0.05 & 0.50 & - . & $\cdots$ & 0.37 & 0.35 & 0.43 & 2.15 & $\cdots$ & - - & $\cdots$ & \\
\hline HR 3994 & 2.15 & 0.22 & 0.39 & 0.17 & 0.54 & $\ldots$ & $\ldots$ & 0.17 & 0.39 & 0.47 & 2.70 & $\ldots$ & $\ldots$ & $\ldots$ & \\
\hline HR 4287 & 2.45 & -0.06 & 0.05 & 0.11 & 0.15 & $\ldots$ & - . & 0.14 & 0.06 & -0.14 & 2.15 & . . & $\ldots$ & ... & \\
\hline HR 4521 & 2.67 & 0.41 & 0.46 & 0.05 & 0.36 & $\cdots$ & $\cdots$ & 0.55 & 0.53 & 0.41 & 2.35 & - - & $\cdots$ & - - & \\
\hline HR 4932 & 2.11 & 0.08 & 0.12 & 0.04 & 0.14 & - - & $\cdots$ & 0.37 & -0.04 & 0.02 & 2.10 & $\cdots$ & $\cdots$ & - . & \\
\hline HR 5270 & 2.51 & -2.60 & $-2.67:$ & -0.07 & & $\cdots$ & $\cdots$ & -2.67 & $\cdots$ & & 1.50 & $\cdots$ & $\cdots$ & $\cdots$ & \\
\hline HR 5340 & 2.95 & -0.42 & -0.46 & -0.04 & -0.43 & $\cdots$ & $\cdots$ & -0.33 & -0.46 & -0.61 & 2.10 & $\ldots$ & $\ldots$ & $\cdots$ & \\
\hline HR 5370 & 2.78 & 0.31 & 0.22 & -0.09 & 0.07 & $\ldots$ & $\ldots$ & 0.23 & 0.36 & 0.20 & 1.65 & $\ldots$ & $\ldots$ & $\ldots$ & \\
\hline HR 5 & 2.27 & 0.00 & -0.08 & -0.08 & -0.12 & $\ldots$ & $\ldots$ & -0.10 & -0.05 & -0.05 & 1.5 & $\ldots$ & $\ldots$ & $\ldots$ & \\
\hline HR 5582 & 2.79 & 0.42 & 0.48 & 0.06 & 0.73 & $\ldots$ & $\cdots$ & 0.33 & 0.42 & 0.43 & 1.60 & $\ldots$ & $\ldots$ & $\ldots$ & \\
\hline HR 5681 & 2.27 & -0.44 & -0.39 & 0.05 & -0.47 & $\cdots$ & $\cdots$ & -0.47 & -0.35 & -0.28 & 2.45 & $\cdots$ & $\cdots$ & $\cdots$ & \\
\hline HR 5744 & 2.61 & 0.12 & 0.08 & -0.04 & -0.08 & $\cdots$ & $\cdots$ & 0.05 & 0.26 & 0.08 & 2.05 & $\cdots$ & $\cdots$ & $\cdots$ & \\
\hline HR 5826 & 3.72 & -0.10 & -0.02 & 0.08 & -0.11 & $\ldots$ & $\ldots$ & -0.25 & 0.07 & 0.21 & 1.00 & $\ldots$ & $\ldots$ & $\cdots$ & \\
\hline HR 5 & 2.58 & 0.25 & 0.23 & -0.02 & 0.36 & $\cdots$ & $\cdots$ & 0.19 & 0.09 & 0.2 & 2.1 & $\ldots$ & $\cdots$ & $\cdots$ & \\
\hline & 2.37 & -0.30 & -0.29 & 0.01 & $\cdots$ & $\cdots$ & $\cdots$ & -0.42 & -0.17 & -0.28 & & $\ldots$ & $\cdots$ & $\cdots$ & \\
\hline HR 5901 & 2.33 & 0.02 & 0.00 & -0.02 & $\ldots$ & $\ldots$ & $\ldots$ & 0.14 & -0.21 & 0.06 & 2.85 & $\cdots$ & $\cdots$ & $\ldots$ & \\
\hline HR 5940 & 2.60 & 0.10 & -0.10 & -0.20 & & $\cdots$ & $\cdots$ & -0.15 & -0.24 & 0.08 & 2.55 & $\cdots$ & $\cdots$ & $\cdots$ & \\
\hline HR 5947 & 2.88 & -0.29 & -0.34 & -0.05 & $\cdots$ & $\cdots$ & $\cdots$ & -0.20 & -0.51 & -0.29 & 1.70 & $\ldots$ & $\cdots$ & $\cdots$ & \\
\hline HR 6014 & 2.26 & 0.13 & 0.10 & -0.03 & $\ldots$ & $\ldots$ & $\ldots$ & 0.20 & 0.00 & 0.11 & & $\ldots$ & $\ldots$ & $\ldots$ & \\
\hline HR 601 & 2.39 & 0.01 & -0.1 & -0.15 & -0.36 & $\ldots$ & $\cdots$ & -0.06 & -0.0 & -0 . & & $\ldots$ & $\cdots$ & $\ldots$ & \\
\hline HR 6103 & 2.23 & 0.01 & -0.07 & -0.0 & & $\ldots$ & $\cdots$ & -0.01 & -0.30 & 0. & 2.3 & $\ldots$ & $\ldots$ & $\ldots$ & \\
\hline HR 6136 & 3.36 & 0.20 & 0.25 & 0.05 & 0.25 & $\ldots$ & $\ldots$ & 0.14 & 0.24 & 0.37 & 1.00 & $\ldots$ & $\ldots$ & $\ldots$ & \\
\hline HR 6159 & 3.64 & -0.13 & -0.19 & -0.06 & 0.08 & $\ldots$ & - . & -0.25 & -0.33 & -0.2 & 1.45 & $\ldots$ & $\ldots$ & $\ldots$ & \\
\hline HR 6 & 2.5 & 0.09 & 0.0 & -0.0 & -0.03 & $\ldots$ & $\ldots$ & 0.11 & 0.2 & -0 . & & $\ldots$ & $\ldots$ & $\ldots$ & \\
\hline HR 6 & 2.17 & -0.05 & -0. & 0.0 & -0.06 & $\ldots$ & $\cdots$ & 0.0 & 0. & -0 . & & $\ldots$ & $\ldots$ & $\cdots$ & \\
\hline HR 6817 & 2.18 & -0.06 & -0 . & -0.0 & 0.00 & $\ldots$ & $\cdots$ & -0.0 & -0.21 & -0. & 3. & $\ldots$ & $\ldots$ & $\ldots$ & \\
\hline HR 6 & 2.59 & 0.07 & 0.04 & -0.03 & $\ldots$ & $\ldots$ & $\ldots$ & 0.07 & -0.06 & 0.10 & 1.7 & $\ldots$ & $\ldots$ & $\ldots$ & \\
\hline HR 71 & 2.45 & -0.09 & 0. & 0.1 & -0.05 & $\ldots$ & $\cdots$ & 0. & 0.0 & -0 . & & $\ldots$ & $\cdots$ & $\cdots$ & \\
\hline HR 714 & & 0.0 & -0 . & -0. & & $\ldots$ & $\ldots$ & -0.0 & -0.0 & 0. & & $\ldots$ & $\ldots$ & $\ldots$ & \\
\hline & 2.3 & 0.17 & 0. & -0.0 & & $\ldots$ & $\ldots$ & -0. & 0.1 & 0. & & $\ldots$ & $\cdots$ & $\cdots$ & \\
\hline $\mathrm{HR} 7317$ & 3.55 & -0.35 & -0.4 & -0.1 & -0.38 & $\ldots$ & $\ldots$ & -0.58 & -0.58 & 0.4 & & $\ldots$ & $\ldots$ & $\ldots$ & \\
\hline & & 0.3 & 0.4 & 0.0 & 0.49 & $\ldots$ & $\cdots$ & 0.75 & 0.3 & 0.3 & 2.4 & $\ldots$ & $\ldots$ & $\ldots$ & \\
\hline & & 0.22 & 0. & -0.1 & -0.04 & $\ldots$ & $\ldots$ & 0.10 & 0.1 & 0.1 & & & $\ldots$ & $\cdots$ & \\
\hline HR 7 & & -0.2 & -0 . & 0. & -0.12 & $\cdots$ & $-\cdots$ & -0.1 & -0.2 & 0.0 & & $\cdots$ & $\ldots$ & $\cdots$ & \\
\hline HR 75 & 2.82 & 0.42 & 0.43 & 0.01 & 0.52 & $\cdots$ & $\cdots$ & 0.38 & 0.3 & 0.4 & 2.15 & $\cdots$ & $\cdots$ & $\cdots$ & \\
\hline HR 78 & 2.01 & -0.35 & -0.2 & 0.08 & -0.22 & $\ldots$ & $\cdots$ & -0.32 & -0.27 & -0.28 & & $\ldots$ & $\ldots$ & $\ldots$ & \\
\hline $\mathrm{HR} 7 \mathrm{~S}$ & 2.16 & -0.14 & -0.26 & -0.1 & -0.26 & $\ldots$ & $\ldots$ & -0.11 & -0.17 & -0.9 & & $\cdots$ & $\ldots$ & $\ldots$ & \\
\hline & 2.4 & & -0 . & & -0.22 & $\ldots$ & $\ldots$ & -0.2 & -0. & & & $\cdots$ & $\cdots$ & $\cdots$ & \\
\hline HR 8 & 2. & -0.13 & -0. & 0.0 & -0.13 & $\ldots$ & $\ldots$ & 0. & 0.0 & -0 . & & $\ldots$ & $\ldots$ & - - & \\
\hline HR 8 & 2.44 & 0.55 & 0.50 & -0.05 & 0.41 & $\ldots$ & $\ldots$ & 0.58 & 0.50 & 0.5 & 3.05 & $\cdots$ & $\ldots$ & $\cdots$ & \\
\hline HD 165195 & 2.67 & -2.70 & -2.62 : & 0.08 : & & $\cdots$ & $\ldots$ & -2.62 & $\ldots$ & $-1.34:$ & 1.20 & $\cdots$ & $\ldots$ & $\cdots$ & \\
\hline HD 199580 & 2.06 & -0.10 & -0.05 & 0.05 & -0.03 & $\ldots$ & $\cdots$ & 0.12 & -0.13 & -0.16 & 3.50 & $\cdots$ & $\cdots$ & $\cdots$ & \\
\hline
\end{tabular}

Field Dwarfs

\begin{tabular}{|c|c|c|c|c|c|c|c|c|c|c|c|c|c|c|c|}
\hline $\begin{array}{l}\text { HD } 1461 \\
\text { HD } 3567\end{array}$ & $\begin{array}{l}1.35 \\
1.33\end{array}$ & $\begin{array}{r}0.33 \\
-1.44\end{array}$ & $\begin{array}{r}0.59 \\
1.70 .\end{array}$ & 0.26 & - . & 0.83 & 0.70 & 0.41 & 0.32 & 0.70 & 4.30 & 4.74 & 0.44 & 4.55 & 4.85 \\
\hline $\begin{array}{l}\text { HD } 3567 \\
\text { HD } 3651\end{array}$ & $\begin{array}{l}1.35 \\
1.89\end{array}$ & $\begin{array}{l}-1.44 \\
-0.18\end{array}$ & $\begin{array}{c}-1.79: \\
0.17\end{array}$ & $\begin{array}{c}-0.35 \text { : } \\
0.35\end{array}$ & $\cdots$ & $\begin{array}{r}-1.79 \\
0.12\end{array}$ & 0.08 & 0.23 & 0.27 & 0.22 & 4.50 & 4.82 & 0.32 & 4.74 & 4.87 \\
\hline HD 4307 & 1.50 & -0.52 & -0.25 & 0.27 & $\ldots$ & $\begin{array}{l}-0.20 \\
-\end{array}$ & -0.44 & -0.05 & -0.31 & -0.07 & 3.93 & $\begin{array}{l}4.02 \\
4.13\end{array}$ & 0.20 & $\begin{array}{l}4.03 \\
4.03\end{array}$ & $\begin{array}{l}4.18 \\
\end{array}$ \\
\hline HD 4628 & 2.15 & & -0.32 & & & -0.48 & -0.45 & -0.40 & -0.16 & -0.16 & 4.64 & 4.35 & -0.29 & 4.16 & 4.47 \\
\hline HD 4614 & 1.48 & -0.19 & -0.69 & -0.50 & $\ldots$ & -0.47 & -0.89 & -1.23 & -0.44 & -0.21 & 4.30 & 3.72 & -0.58 & 3.46 & 3.88 \\
\hline HD 10307 & 1.39 & 0.12 & 0.12 & 0.00 & $\cdots$ & 0.08 & 0.37 & -0.12 & -0.26 & 0.33 & 4.30 & 4.48 & 0.18 & 4.49 & 4.47 \\
\hline HD 10476 & 2.03 & -0.20 & -0.10 & 0.10 & $\ldots$ & 0.23 & -0.44 & 0.04 & 0.20 & -0.13 & 4.60 & 4.55 & -0.05 & 4.34 & 4.67 \\
\hline HD 10700 & 1.82 & -0.37 & -0.30 & 0.07 & $\ldots$ & 0.64 & -0.33 & -0.56 & & -0.64 & 4.56 & 4.62 & 0.06 & 4.60 & 4.63 \\
\hline HD 10780 & 1.69 & 0.36 & 0.12 & -0.24 & $\ldots$ & 0.10 & -0.02 & 0.22 & 0.21 & 0.19 & 4.60 & 4.55 & -0.05 & 4.28 & 4.71 \\
\hline HD 13043 & 1.44 & 0.07 & 0.05 & -0.02 & & 0.10 & 0.02 & -0.05 & -0.07 & 0.30 & 4.01 & 4.17 & 0.16 & 3.97 & 4.30 \\
\hline HD 13783 & 1.65 & -0.55 & -0.06 : & 0.49 : & & -0.06 & & & & & & & & & \\
\hline HD 14802 & 1.57 & -0.17 & -0.27 & -0.10 & $\ldots$ & -0.11 & -0.46 & -0.11 & -0.28 & -0.21 & 3.92 & 3.55 & -0.37 & 3.59 & 3.52 \\
\hline HD 19373 & 1.36 & 0.09 & 0.17 & 0.08 & $\cdots$ & 0.03 & 0.51 & -0.05 & -0.10 & 0.16 & 4.10 & 4.32 & 0.22 & 4.43 & 4.25 \\
\hline HD 19445 & 1.39 & -2.07 & -1.74 & 0.33 & & -2.27 & -1.87 & -1.82 & -1.06 & & 4.23 & 4.25 & 0.02 & 4.56 & 4.06 \\
\hline HD 20630 & 1.57 & 0.23 & -0.06 & -0.29 & $\ldots$ & -0.14 & -0.28 & 0.21 & 0.20 & -0.14 & 4.4 & 4.3 & -0.8 & 3. & 4.54 \\
\hline HD 22484 & 1.36 & 0.02 & 0.04 & 0.02 & & -0.09 & $\begin{array}{r}-0.20 \\
0.19\end{array}$ & 0.01 & 0.19 & $\begin{array}{l}-0.14 \\
-0.26\end{array}$ & 3.8 & 3.5 & 0.1 & 4.00 & $\begin{array}{l}4.34 \\
3.92\end{array}$ \\
\hline HD 22879 & 1.45 & -0.85 & -0.60 & 0.25 & & -0.76 & -0.69 & -0.70 & -0.58 & -0.26 & 4.27 & $\begin{array}{l}3.95 \\
4.34\end{array}$ & 0.07 & 4.46 & 4.26 \\
\hline HD 23439A & 1.99 & -1.02 & -1.24 & -0.22 & & -1.01 & -0.75 & -1.95 & -1.59 & -0.70 & 4.63 & 4.94 & 0.31 & 4.96 & 4.92 \\
\hline HD $23439 \mathrm{~B}$ & 2.32 & -1.05 & -0.95 & 0.10 & & -2.88 & -0.55 & -0.96 & -1.39 & $-0.74:$ & 4.65 & 4.99 & 0.34 & 5.25 & 4.82 \\
\hline HD 24451 & 2.80 & & 0.03 & & & -0.09 & & 0.18 & -0.11 & & 4.6 & & 0.34 & & \\
\hline HD 25329 & 2.26 & -1.61 & -1.36 & 0.25 & $\ldots$ & -1.0 & -1.17 & -1.2 & -1.81 & -1.19 . & 4. & 4.83 & 0.06 & 539 & 450 \\
\hline HD 26965 & 2.02 & -0.03 & -0.17 & -0.14 & & 0.8 & -0.11 & -0.5 & -0.59 & -0.22 & 4. & $\begin{array}{l}4.0 \\
4.6\end{array}$ & 0.14 & 4.44 & 4.80 \\
\hline HD 27561 & .95 & 0.15 & 0.04 & -0.11 & $\ldots$ & 0.03 & 0.03 & 0.12 & -0.14 & 0.16 & 4.18 & 4.13 & -0.05 & 4.08 & 4.16 \\
\hline HD 28068 & 1.55 & 0.15 & -0.11 & -0.26 & $\ldots$ & -0.73 & 0.03 & -0.40 & 0.08 & 0.16 & 4.2 & 4.0 & -0.21 & 4.11 & 3.94 \\
\hline HD 28344 & 1.40 & 0.15 & 0.07 & -0.08 & $\ldots$ & -0.36 & 0.14 & 0.08 & -0.05 & 0.33 & 4.35 & 4.23 & -0.12 & 4.34 & 4.16 \\
\hline HD 30455 & 1.51 & -0.18 & -0.08 : & 0.10 : & $\ldots$ & -0.08 & $\ldots$ & $\cdots$ & $\ldots$ & $\cdots$ & & $\ldots$ & $\cdots$ & $\cdots$ & $\cdots$ \\
\hline HD 3 & 1.53 & -0.26 & $-0.31:$ & $-0.05:$ & & -0.3 & & & & & & & & & \\
\hline HD 30652 & 1.08 & 0.14 & 0.14 & 0.00 & $\ldots$ & -0.04 & 0.01 & 0.47 & 0.30 & 0.04 & 4.29 & 4.14 & -0.15 & 4.18 & 4.11 \\
\hline HD 34411 & 1.43 & 0.19 & 0.0 & -0.13 & $\ldots$ & -0.0 & 0.12 & 0.08 & 0.03 & 0.02 & 4.20 & 4.15 & -0.05 & 4.14 & 4.16 \\
\hline HD 36 & & & 0.09 & & & 0.32 & & 0.02 & 0.16 & & 4.61 & & & & \\
\hline HD 3 & 1 & -0.07 & -0.17 & -0.10 & & -0.24 & -0.11 & -0.06 & -0.09 & -0.44 & 4.17 & 4.09 & -0.08 & 4.1 & 4.06 \\
\hline HD 3 & 1.44 & 0.08 & -0.16 & -0.24 & & -0.30 & -0.05 & -0.11 & -0.36 & -0.11 & 4.40 & 4.29 & -0.11 & 4.28 & 4.30 \\
\hline & 1.4 & 0.15 & -0.27 : & -0.42 : & & -0.2 & & & & & $\ldots$ & $\cdots$ & & & \\
\hline HD 5 & 1.53 & -0.33 & -0.48 & -0.15 & $\cdots$ & -0.67 & -0.50 & -0.57 & -0.69 & 0.00 & 3.90 & 3.75 & -0.15 & 3.71 & 3.77 \\
\hline HD 64606 & 2.03 & -0.92 & $-0.94:$ & -0.02 : & & -0.94 & & & & & & & & & \\
\hline HD 74 & 2.20 & & -0.01 & & $\ldots$ & -1.18 & 0.09 & -0.06 & -0.08 & -0.06 & 4.63 & 4.80 & 0.17 & 4.71 & 4.85 \\
\hline HD 7 & 1.74 & 0.13 & $-0.17:$ & -0.30 : & & & & & & & & & & & \\
\hline HD 84937 & 1.19 & -2.10 & -1.87 & 0.23 & $\ldots$ & -2.10 & -2.03 & -1.44 & $\ldots$ & $-2.04:$ & 4.61 & 4.65 & 0.04 & 4.72 & 4.61 \\
\hline HD 86728 & 1.46 & 0.16 & 0.30 & 0.14 & $\ldots$ & 0.27 & 0.44 & 0.23 & 0.15 & 0.29 & 4.25 & 4.54 & 0.29 & 4.47 & 4.58 \\
\hline
\end{tabular}


TABLE 5-Continued

\begin{tabular}{|c|c|c|c|c|c|c|c|c|c|c|c|c|c|c|c|}
\hline \multirow[t]{2}{*}{ Name } & \multirow[t]{2}{*}{$V-K$} & \multirow[t]{2}{*}{$\mathrm{Fe}_{i n}$} & \multirow{2}{*}{$\mathrm{Fe}_{\text {out }}$} & \multirow[t]{2}{*}{$\Delta \mathrm{Fe}$} & \multicolumn{6}{|c|}{$\mathrm{Fe}_{\text {out }}$ by Feature } & \multirow{2}{*}{$g_{i n}$} & \multirow{2}{*}{$g_{\text {out }}$} & \multirow[t]{2}{*}{$\Delta g$} & \multicolumn{2}{|c|}{$g_{\text {out }}$ by Feature } \\
\hline & & & & & $\mathrm{CN}$ & $\bar{G}$ & $\mathrm{Mg}_{2}$ & Fe52 & $\mathrm{Fe} 53$ & $\mathrm{Na} \mathrm{D}$ & & & & $\mathrm{Mg}_{2}$ & $\mathrm{Mg} b$ \\
\hline \multicolumn{16}{|c|}{ Field Dwarfs (continued) } \\
\hline HD 90508 & 1.50 & -0.23 & $-0.17:$ & $0.06:$ & $\cdots$ & -0.17 & - . & $\cdots$ & $\cdots$ & $\ldots$ & $\cdots$ & $\cdots$ & $\cdots$ & $\ldots$ & $\cdots$ \\
\hline $\begin{array}{l}\text { HD } 98230 \\
\text { HD } 101501\end{array}$ & $\begin{array}{l}1.54 \\
1.74\end{array}$ & -0.12 & 0.11: & 0.23: & $\cdots$ & $\begin{array}{r}0.11 \\
-0.34\end{array}$ & $\cdots$ & $\because-0$ & $\cdots$ & $\cdots$ & $\cdots$ & $\cdots$ & $\cdots$ & $\cdots$ & $\cdots$ \\
\hline HD 102870 & 1.27 & 0.18 & 0.24 & 0.06 & $\ldots$ & 0.12 & 0.35 & 0.24 & $\begin{array}{l}-0.09 \\
0.09\end{array}$ & $\begin{array}{r}-0.20 \\
0.29\end{array}$ & $\begin{array}{l}4.00 \\
4.05\end{array}$ & $\begin{array}{l}4.35 \\
4.10\end{array}$ & 0.05 & $\begin{array}{l}4.30 \\
4.19\end{array}$ & $\begin{array}{l}4.34 \\
4.05\end{array}$ \\
\hline HD 103095 & 2.03 & -1.32 & -1.28 & 0.04 & $\cdots$ & -1.35 & -1.13 & -1.51 & & -1.70 & 4.70 & 4.68 & -0.02 & 4.96 & 4.52 \\
\hline HD 106516 & 1.22 & -0.82 & -0.59 & 0.23 & $\ldots$ & -1.04 & -0.34 & -0.28 & -1.08 & -0.53 & 4.24 & 5.05 & 0.81 & $\begin{array}{l}4.90 \\
4.76\end{array}$ & 5.19 \\
\hline HD 108177 & 1.30 & -1.73 & -1.78 & -0.05 & $\cdots$ & -1.80 & -1.96 & -1.44 & & & 5.04 & 4.60 & -0.44 & 4.70 & 4.53 \\
\hline HD 110897 & 1.41 & -0.36 & -0.62 & -0.26 & $\ldots$ & -0.52 & -0.46 & -0.63 & -1.14 & -0.47 & 4.50 & 4.32 & -0.18 & 4.39 & 4.27 \\
\hline HD 114710 & 1.37 & 0.15 & -0.05 & -0.20 & $\cdots$ & -0.32 & -0.05 & -0.01 & 0.02 & 0.05 & 4.34 & 4.17 & -0.17 & 4.16 & 4.17 \\
\hline HD 114762 & 1.53 & -0.75 & $-0.96:$ & $-0.21:$ & - . & -0.96 & $\cdots$ & $\cdots$ & $\ldots$ & & & & & & \\
\hline HD 115043 & 1.32 & -0.10 & 0.00 : & 0.10 : & $\ldots$ & 0.00 & 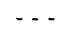 & $\ldots$ & $\ldots$ & $\ldots$ & $\ldots$ & $\ldots$ & & & \\
\hline HD 115617 & 1.57 & 0.22 & -0.03 & -0.25 & $\cdots$ & -0.13 & -0.04 & 0.06 & 0.17 & -0.24 & 4.52 & 4.40 & -0.12 & 4.30 & 4.46 \\
\hline HD 117176 & 1.74 & -0.21 & -0.17 & 0.04 & $\cdots$ & -0.06 & -0.21 & -0.08 & -0.17 & -0.27 & 3.80 & 3.91 & 0.11 & 3.80 & 3.98 \\
\hline HD 120136 & 1.07 & 0.21 & 0.62 : & $0.41:$ & $\cdots$ & 0.62 & & & & $\ldots$ & & & & $\cdots$ & \\
\hline HD 131977 & 2.65 & 0.01 & 0.10 & 0.09 & $\ldots$ & $\cdots$ & & 0.08 & 0.12 & & 4.70 & & & & \\
\hline HD 132142 & 1.97 & -0.55 & -0.19 & 0.36 & $\ldots$ & 0.81 & -0.32 & -0.56 & 0.25 & -0.72 & 4.60 & 4.87 & 0.27 & 4.84 & 4.89 \\
\hline HD 134439 & 2.02 & -1.40 & -1.45 & -0.05 & $\ldots$ & -1.78 & -1.35 & -1.30 & -1.58 & & 4.51 & 4.41 & -0.10 & 4.59 & 4.30 \\
\hline HD 140283 & 1.53 & -2.45 & -2.26 & 0.19 & $\ldots$ & -2.88 & -2.92 & -1.68 & -1.24 & $-1.96:$ & 4.24 & 3.57 & -0.67 & 3.21 & 3.79 \\
\hline HD 142373 & 1.49 & -0.38 & -0.55 & -0.17 & $\ldots$ & -0.36 & -0.60 & -1.13 & -0.55 & -0.01 & 3.90 & 3.94 & 0.04 & 3.64 & 4.11 \\
\hline HD 142860 & 1.20 & -0.25 & -0.34 & -0.09 & $\cdots$ & -0.44 & -0.46 & 0.06 & -0.32 & -0.47 & 4.27 & 4.08 & -0.19 & 4.05 & 4.09 \\
\hline HD 143761 & 1.40 & -0.17 & 0.27 & 0.44 & - - & 0.32 & 0.59 & -0.11 & 0.06 & 0.25 & 3.90 & 4.33 & 0.43 & 4.53 & 4.21 \\
\hline HD 144872 & 2.23 & & -0.18 & & $\ldots$ & & -0.30 & -0.12 & -0.12 & -0.10 & 4.65 & 4.47 & -0.18 & 4.3 & 4.54 \\
\hline HD 145675 & 1.73 & 0.27 & 0.53 & 0.26 & $\ldots$ & 0.15 & 0.85 & 0.51 & 0.35 & 0.40 & 4.5 & $\begin{array}{l}4.41 \\
4.98\end{array}$ & $\begin{aligned}-0.1 \\
0.4\end{aligned}$ & & 5.00 \\
\hline HD 149661 & 1.91 & 0.01 & -0.02 & -0.03 & $\cdots$ & -0.57 & -0.15 & 0.36 & 0.11 & 0.11 & 4.50 & 4.52 & 0.02 & 4.34 & 4.62 \\
\hline HD 152792 & 1.54 & -0.38 & $-0.16:$ & $0.22:$ & $\cdots$ & -0.16 & & & & & & & & & \\
\hline HD 157089 & 1.48 & -0.56 & -0.47 & 0.09 & $\cdots$ & -0.22 & -0.63 & -0.68 & -0.49 & -0.15 & 4.38 & 4.21 & -0.17 & 4.30 & 4.16 \\
\hline HD 157214 & 1.52 & -0.43 & -0.17 & 0.26 & $\ldots$ & 0.28 & -0.40 & -0.36 & 0.16 & -0.22 & 4.20 & 4.29 & 0.09 & 4.24 & 4.31 \\
\hline HD 160693 & 1.49 & -0.69 & 0.26 : & 0.95 : & $\ldots$ & 0.26 & 7.70 & & & -0.22 & 4.20 & - & 0.07 & & \\
\hline & 2.17 & -0.25 & -0.30 & -0.05 & $\ldots$ & & -0.40 & -0.35 & -0.14 & -0.25 & 4.65 & 4.54 & -0.11 & 4.49 & 4.57 \\
\hline HD 170153 & 1.38 & -0.33 & -1.01 : & -0.68 : & $\cdots$ & -1.01 & & & & & & & & & \\
\hline HD 185144 & 1.91 & -0.24 & -0.33 & -0.09 & $\cdots$ & -0.34 & -0.49 & -0.22 & -0.23 & -0.26 & 4.50 & 4.36 & -0.14 & 4.23 & 4.44 \\
\hline HD 186408 & 1.43 & 0.14 & 0.2 & 0.14 & $\cdots$ & 0.28 & $\cdots$ & $\cdots$ & $\ldots$ & $\cdots$ & $\cdots$ & $\ldots$ & & ... & \\
\hline HD 1864 & 1.55 & 0.8 & -0.0 & -0.1 & $\ldots$ & -0.06 & & $\ldots$ & $\ldots$ & $\ldots$ & $\ldots$ & $\ldots$ & $\ldots$ & $\ldots$ & $\ldots$ \\
\hline HD 187691 & 1.28 & 0.12 & $-0.26:$ & -0.3 & $\ldots$ & -0.26 & $\ldots$ & $\cdots$ & $\ldots$ & $\ldots$ & $\ldots$ & $\cdots$ & & $\cdots$ & $\ldots$ \\
\hline HD 187923 & 1.51 & 0.06 & $0.28:$ & $0.22:$ & $\ldots$ & 0.28 & $\ldots$ & $\ldots$ & $\ldots$ & $\ldots$ & $\ldots$ & $\ldots$ & . & $\ldots$ & $\ldots$ \\
\hline HD 193901 & 1.50 & -1.22 & $-1.38:$ & -0.16 & $\cdots$ & -1.38 & $\cdots$ & $\cdots$ & $\cdots$ & $\cdots$ & $\cdots$ & $\ldots$ & $\cdots$ & $\ldots$ & $\ldots$ \\
\hline HD 1956 & 1.4 & -1.12 & $-1.17:$ & $-0.05:$ & $\cdots$ & -1.17 & & $\cdots$ & $\ldots$ & $\cdots$ & - . & $\ldots$ & $\ldots$ & $\ldots$ & $\cdots$ \\
\hline HD 1970 & 1.43 & -0.08 & -0.0 & -0.01 & $\ldots$ & -0.38 & -0.14 & -0.10 & -0.08 & 0.19 & 4.24 & 4.33 & 0.09 & 4.18 & 4.42 \\
\hline HD $2 C$ & 2.92 & & 0.1 & $\cdots$ & $\ldots$ & $\cdots$ & & 0.21 & 0.04 & & 4.63 & & & & \\
\hline HD 20 & 1.40 & -1.23 & -0.9 & 0.26 & - - & -0.80 & -1.02 & -1.21 & -0.86 & -0.49 : & 4.40 & 4.52 & 0.12 & 4.67 & 4.43 \\
\hline HD 205153 & 1.30 & -0.06 & 0.22 & 0.28 & & 0.54 & -0.12 & 0.11 & 0.19 & 0.76 & 3.70 & 4.03 & 0.33 & 3.64 & 4.23 \\
\hline HD 2 & 1.4 & -1.24 & -0.5 & 0.70 : & & -0.54 & & & & & & & & & \\
\hline & 1.33 & -0.8 & -0. & 0.32 & & -0.67 & -0.73 & -0.54 & -0.33 & -0.09 & 4.28 & 4.43 & 0.15 & 4.38 & 4.46 \\
\hline HD 2100 & 1.10 & -0.10 & $-0.41:$ & -0.31 : & & -0.41 & & & & $\ldots$ & & & & & \\
\hline HD 2156 & 1.34 & -0.40 & -0.44 & -0.04 & - & -0.83 & -0.62 & -0.28 & -0.08 & -0.37 & 3.83 & 3.59 & -0.24 & 3.57 & 3.61 \\
\hline HD 2178 & 1.3 & -0.31 & -0.0 & 0.25 & $\ldots$ & -0.29 & -0.22 & 0.01 & 0.02 & 0.25 & 4.08 & 4.2 & 0.20 & 4.18 & 4.35 \\
\hline HD 219134 & 2.34 & 0.0 & -0.0 & -0.05 & $\ldots$ & $\ldots$ & -0.28 & 0.09 & 0.26 & -0.03 & 4.65 & 4.41 & -0.24 & 4.35 & 4.45 \\
\hline HD 219617 & 1.35 & -1.68 & $-1.57:$ & $0.11:$ & & -1.57 & & & & $\ldots$ & $\cdots$ & & & & \\
\hline HD 222368 & 1.36 & -0.33 & -0.40 & -0.07 & $\cdots$ & -0.60 & -0.61 & -0.48 & -0.11 & -0.09 & 3.92 & 3.45 & -0.47 & 3.59 & 3.37 \\
\hline HD 224930 & 1.84 & -0.90 & $\begin{array}{l}-0.83 \\
-0.80\end{array}$ & 0.07 & $\ldots$ & -1.02 & $\begin{array}{l}-0.78 \\
-0.78\end{array}$ & -1.21 & -1.01 & $\begin{array}{l}-0.19 \\
-0.19\end{array}$ & 4.36 & 4.46 & 0.10 & 4.51 & 4.43 \\
\hline BD +174708 & 1.38 & -1.90 & -2.12 : & -0.22 : & $\cdots$ & -2.12 & $\cdots$ & $\cdots$ & $\cdots$ & $\ldots$ & $\cdots$ & $\cdots$ & $\cdots$ & $\cdots$ & $\ldots$ \\
\hline
\end{tabular}

NOTES. $-\mathrm{Fe} \equiv[\mathrm{Fe} / \mathrm{H}]$ and $g \equiv \log g$.

$[\mathrm{Fe} / \mathrm{H}]_{\text {out }}$ and $(\log g)_{\text {out }}$ are weighted averages of feature estimates, with weights based on the rms errors in Tables 8 and 9 , as follows:

$$
\begin{array}{ll}
{[\mathrm{Fe} / \mathrm{H}]_{\text {out }}:} & \text { Giants: } \mathrm{CN}=\mathrm{Mg}_{2}=\mathrm{Fe} 52=\mathrm{Fe} 53=\mathrm{Na} \mathrm{D}=1.0 . \\
(\log g)_{\text {out }}: & \text { Dwarfs: } \mathrm{G}=0.7, \mathrm{Mg}_{2}=1.8, \mathrm{Fe} 52=\mathrm{Fe} 53=\mathrm{Na} \mathrm{D} \\
& \text { Giants: } \mathrm{Mg}_{2}=1.0, \mathrm{Mg} b=0.5 . \\
& \text { Dwarfs: } \mathrm{Mg}_{2}=0.6, \mathrm{Mg} b=1.0 .
\end{array}
$$

The following field $\mathrm{K}$ giants have had $[\mathrm{Fe} / \mathrm{H}]_{\text {in }}$ revised from Paper II:

\begin{tabular}{lcc}
\multicolumn{1}{c}{ Star } & $\mathrm{Fe}_{\text {old }}$ & $\mathrm{Fe}_{\text {new }}$ \\
\hline HR 296 & -0.14 & -0.48 \\
HR 2503 & -0.03 & +0.23 \\
HR 5826 & -0.43 & -0.10 \\
HR 5947 & -0.08 & -0.29 \\
HR 6136 & +0.35 & +0.20 \\
HR 7148 & +0.07 & -0.09 \\
HR 7430 & -0.70 & -0.20
\end{tabular}

Missing output values mean input $[\mathrm{Fe} / \mathrm{H}]$ or $\log g$ not available, line index not measured, output value imaginary, or $V-K$ and/or $Z$ outside region of validity.

Metallicities for dwarfs without $[\mathrm{Fe} / \mathrm{H}]_{\text {in }}$ are assumed to be 0.0 .

Since gravities have been estimated from $\mathrm{Mg}_{2}$ for field $\mathrm{K}$ giants, no output gravities or metallicities can be returned from $\mathrm{Mg}_{2}$ or $\operatorname{Mg} b$ (see text).

$[\mathrm{Fe} / \mathrm{H}]_{\text {out }}$ not returned for dwarfs from $G$-band unless $V-K \leq 2.15$.

$\mathrm{Na} D$ has been corrected for interstellar absorption as described in $\S 4.5$. Values with colons not used in mean $[\mathrm{Fe} / \mathrm{H}]_{\mathrm{out}}$.

Expression numbers in Table 6 used to derive output metallicities and gravities: 
TABLE 5-Continued

\begin{tabular}{lcc} 
Feature & Dwarfs & Giants \\
\hline $\mathrm{CN}$ & - & - \\
$\mathrm{G}$ & 3 & - \\
$\mathrm{Mg}_{2}$ & 6 & 6 \\
$\mathrm{Mg} b$ & 8 & 8 \\
$\mathrm{Fe} 52$ & 9 & 9 \\
$\mathrm{Fe} 53$ & 10 & 10 \\
$\mathrm{Na} \mathrm{D}$ & 11 & 11
\end{tabular}

For dwarfs lacking input $\log g$, only $G$-band metallicity estimates are shown, for consistency with Table 6 . However, better metallicity estimates could be obtained by assuming typical gravity for $V-K$ from Fig. 3 .

anomalously low also. We therefore cannot use the field $K$ giants to estimate the errors of the fit in either $\mathrm{Mg}_{1}$ or $\mathrm{Mg}_{2}$.

The resultant final fitting functions for $\mathrm{Mg}_{2}$ are given in Table 6, which summarizes the functions for all the indices together with their regions of validity. Residuals, $\Delta \mathrm{Mg}_{2}$, for each star about the fit are shown in Figure $4 b$. As expected, the field $\mathrm{K}$ giants fit too well, reflecting the fact that their gravities have been deduced from the same data. The dwarfs and cluster stars are a truer measure of the real scatter. The dwarfs show no systematic trend with temperature but do show larger scatter at cool temperatures. This is due mainly to small errors in the $V-K$ 's coupled with the steep gradient of $\mathrm{Mg}_{2}$ along the lower main sequence.

The cluster stars show scatter but, aside from NGC 188, little systematic trend with temperature. The stars in NGC 188 ( angles) deviate systematically with $V-K$ color, and show similar deviations in $\mathrm{Mg}_{2}$ and $\mathrm{Mg} b$, as discussed below. The stars in M71 appear to scatter more, probably due to higher observational errors. Three cool giants in M71 (pentagons) show markedly positive residuals that are much larger than the expected errors.

Leaving out all field $\mathrm{K}$ giants and dwarfs cooler than $V-$ $K=1.70$, we find an rms fitting residual of 0.023 mag averaged over all stars. This is significantly larger than the observational error of 0.011 mag per star (see Table 1, which compares observational errors with fitting errors for all indices). Some part of this excess is due to errors in the input gravities, metallicities, and $V-K$ 's, but real deviations also seem to be present, especially in NGC 188. This pattern in which the fitting errors moderately exceed the observational errors is repeated in most of the indices.

Qualitatively, the behavior of the $\mathrm{Mg}_{2}$ index here is similar to that seen in the smaller sample of giants studied in Paper II. However, the calibration versus $V-K$ is now much better determined, being tied down firmly at the hot end by dwarfs. Furthermore, the gravity effects, which were merely noted in Paper II, are now modeled quantitatively. A comparison between the fitting function for $\mathrm{Mg}_{2}$ and Mould's (1978) theoretical $\mathrm{Mg}$ indices is given in Appendix C. A discussion of $\mathrm{Mg} / \mathrm{Fe}$ in elliptical galaxies compared to solar neighborhood stars is given by Worthey et al. (1992), based on this fitting function.

$$
\text { 4.2. } \mathrm{Mg}_{1}
$$

The $\mathrm{Mg}_{1}$ index resembles $\mathrm{Mg}_{2}$ but is offset to bluer wavelengths and measures only $\mathrm{MgH}$ with no contribution from the $\mathrm{Mg} b$ lines. The gravity and metallicity dependences are simi- lar to $\mathrm{Mg}_{2}$. Figures $5 a-5 d$ repeat the same panels shown for $\mathrm{Mg}_{2}$. In fitting this and all subsequent indices, we have used the field $\mathrm{K}$ giant gravities derived from $\mathrm{Mg}_{2}$. We achieved a substantially better fit to $\mathrm{Mg}_{1}$ by using a zero constant term in the exponential form. This required omitting a few stars with negative index values (see Fig. $5 a$ ), which introduced a very small, positive bias for hot dwarfs. However the error is only a few thousandths of a magnitude, and the rest of the fit is improved.

The errors and scatter are generally comparable to $\mathrm{Mg}_{2}$, including the systematic trend in NGC 188 . Leaving out field K giants and dwarfs with $V-K>1.70$ as above, we find an rms fitting residual of $0.017 \mathrm{mag}$ in $\mathrm{Mg}_{1}$ compared to an observational error of $0.010 \mathrm{mag}$ per star (see Table 1). The fitting error thus again slightly exceeds the observational error.

\section{3. $\mathrm{Mg} b 5177$}

The results of fitting $\mathrm{Mg} b 5177$ are shown in Figures $6 a-6 d$. There are no strong systematics in any of the clusters, although the three cool giants in M71 are systematically high again here, and the scatter in NGC 188 is large and perhaps systematically negative. The rms residual using all stars is $0.42 \AA$, compared to an observational error of $0.32 \AA$.

The systematic deviations of $\mathrm{Mg}_{1}, \mathrm{Mg}_{2}$, and $\mathrm{Mg} b$ in NGC 188 do not show up in any other index. Hence they cannot be cured by altering the adopted $V-K$ 's as errors would then crop up in the other indices. The alternative cluster parameters given by Twarog \& Anthony-Twarog (1989) increase the discrepancy slightly, but the difference is not significant. The dwarfs were observed on the same run as the giants, for which no systematic residuals are seen, so observational error is not likely. This problem will be revisited in a future paper with additional cluster data. At present, the cause of the effect is unknown.

The gravity polynomial, $p(V-K, g)$, for $\mathrm{Mg} b 5177$ is strongly quadratic in $\log g$, with the result that, at a fixed value of $(V-K, \operatorname{Mg} b)$, there are two possible roots for $\log g$ (see Fig. $6 c)$. In other words, the surface represented by $p(V-K, g)$ is double-valued in projection, and the isogravity contours wrap around on themselves. Only one of these roots is physically meaningful (in this case the higher one), and thus only one branch of $p(V-K, g)$ is plotted in Figure $6 c$. The lower envelope of the contours is where the surface folds over. According to this mathematical model for the index, stars below this envelope must either be scattered there by observational errors or have erroneous input values of $V-K$ and/or $Z$. Either is 


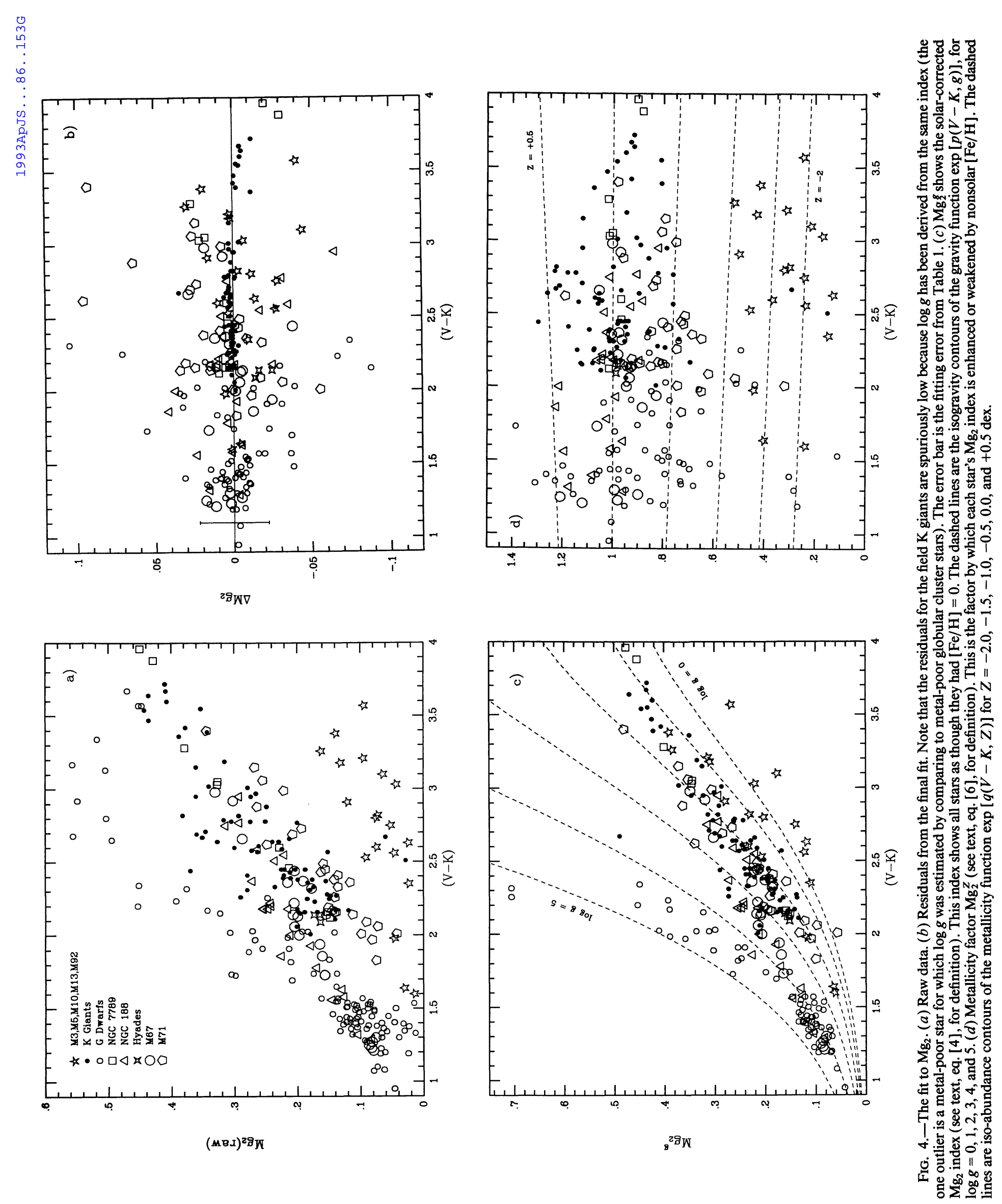


TABLE 6

FITTING FUNCTIONS FOR ABSORPTION INDICES

\begin{tabular}{|c|c|}
\hline Expression $^{a}$ & Region of Validity ${ }^{b}$ \\
\hline 1) $\mathrm{CN}=\exp \left[-3.9439+1.1782(V-K)+0.23704(V-K)^{2}+0.16896(Z)-0.14245(V-K)^{3}\right]-0.175$ & $\begin{array}{l}\text { Dwarfs with } V-K<4.00 \text { and } \\
\text { subgiants with } V-K<1.90 \text {; all }[\mathrm{Fe} / \mathrm{H}] \text {. }\end{array}$ \\
\hline 2) $\mathrm{CN}=-3.9717+4.1365(V-K)-0.032893(\log g)-1.2873(V-K)^{2}+0.24904(Z)+0.12811(V-K)^{3}$ & $\begin{array}{l}\text { Giants with } 1.90<V-K<4.00 \\
\text { and }[\mathrm{Fe} / \mathrm{H}]>-1.00\end{array}$ \\
\hline 3) $\mathrm{G}=-14.882+24.259(V-K)-8.8667(V-K)^{2}+4.2278(Z)-1.6691(Z)(V-K)+0.98914(V-K)^{3}$ & $\begin{array}{l}\text { Dwarfs with } V-K<4.00 \text { and } \\
\text { subgiants with } V-K<1.90 \text {. } \\
Z \text {-terms } \equiv 0 \text { for } V-K>2.50 \text {. }\end{array}$ \\
\hline 4) $\mathrm{G}=-9.9592+16.680(V-K)-5.500(V-K)^{2}+0.59519(V-K)^{3}$ & $\begin{array}{l}\text { All giants with }[\mathrm{Fe} / \mathrm{H}]>-0.75 \text {, } \\
\text { plus dwarfs with }[\mathrm{Fe} / \mathrm{H}]>-0.75 \\
\text { and } V-K<1.90 \text {. Weak sensitivity } \\
\text { to }[\mathrm{Fe} / \mathrm{H}] \text { is ignored. }\end{array}$ \\
\hline 5) $\begin{aligned} \mathrm{H} \beta= & 8.2261-5.9295(V-K)+0.52968(\log g)-0.048352(\log g)^{2}-0.23695(\log g)(V-K) \\
& +1.8169(V-K)^{2}+0.078611(Z)+0.0012346(Z)^{2}+0.020725(Z)(V-K)-0.19721(V-K)^{3}\end{aligned}$ & $\begin{array}{l}\text { All giants, plus dwarfs with } \\
V-K<3.00 \text {. }\end{array}$ \\
\hline 6) $\begin{aligned} \mathrm{Mg}_{2} & =\exp \left[-6.7205+2.5736(V-K)+0.19790(\log g)+0.038509(\log g)^{2}-0.017018(\log g)(V-K)\right. \\
& \left.-0.27692(V-K)^{2}+0.39669(Z)-0.09902(Z)^{2}+0.042443(Z)(V-K)\right]+0.00\end{aligned}$ & All giants, plus dwarfs with $V-K<2.50$. \\
\hline 7) $\begin{aligned} \mathrm{Mg}_{1} & =\exp \left[-10.851+4.5475(V-K)+0.057119(\log g)^{2}+0.05841(\log g)(V-K)-0.55440(V-K)^{2}\right. \\
& \left.+0.34771(Z)-0.09911(Z)^{2}+0.12246(Z)(V-K)\right]+0.00\end{aligned}$ & All giants, plus dwarfs with $V-K<2.50$. \\
\hline 8) $\begin{aligned} \mathrm{Mg} b & =9.6316-3.7907(V-K)-4.7477(\log g)+0.49454(\log g)^{2}+1.3395(\log g)(V-K) \\
& +0.59829(V-K)^{2}+0.43842(Z)+0.073983(Z)^{2}+0.33970(Z)(V-K)\end{aligned}$ & All giants, plus dwarfs with $V-K<2.50$. \\
\hline 9) $\begin{aligned} \text { Fe } 5270 & =-0.53359+2.6591(V-K)-0.84129(\log g)+0.11305(\log g)^{2}+0.13802(\log g)(V-K) \\
& -0.34787(V-K)^{2}+0.83829(Z)+0.14292(Z)^{2}+0.24984(Z)(V-K)\end{aligned}$ & All giants, plus dwarfs with $V-K<3.00$. \\
\hline 10) $\begin{aligned} \text { Fe } 5335 & =+0.30336+1.0836(V-K)-0.74675(\log g)+0.10428(\log g)^{2}+0.17981(\log g)(V-K) \\
& +0.0032477(V-K)^{2}+1.0258(Z)+0.36465(Z)^{2}+0.28420(Z)(V-K)\end{aligned}$ & All giants, plus dwarfs with $V-K<3.00$. \\
\hline 11) $\begin{aligned} \mathrm{Na} \mathrm{D} & =\exp \left[1.4959-0.06221(V-K)-1.3125(\log g)+0.14314(\log g)^{2}+0.30199(\log g)(V-K)\right. \\
& \left.+0.027288(V-K)^{2}+0.73585(Z)+0.15349(Z)^{2}+0.09924(Z)(V-K)\right]+0.00\end{aligned}$ & $\begin{array}{l}\text { All giants, plus dwarfs with } V-K<2.50 \text {; } \\
Z \text { calibration uncertain for }[\mathrm{Fe} / \mathrm{H}]<-1.00 \text {. }\end{array}$ \\
\hline 12) $\begin{aligned} \mathrm{TiO}_{1} & =\exp \left[-5.0842+2.3258(V-K)-1.1664(V-K)^{2}+0.31719(Z)+0.072825(Z)^{2}\right. \\
& \left.+0.011684(Z)(V-K)+0.20124(V-K)^{3}\right]-0.015\end{aligned}$ & All giants, plus dwarfs with $V-K<4.00$. \\
\hline 13) $\begin{aligned} \mathrm{TiO}_{2} & =\exp \left[-3.5741-0.20518(V-K)-0.092837(V-K)^{2}-0.57917(Z)-0.072495(Z)^{2}\right. \\
& \left.+0.29949(Z)(V-K)+0.033314(V-K)^{3}\right]-0.030\end{aligned}$ & All giants, plus dwarfs with $V-K<4.00$. \\
\hline
\end{tabular}

${ }^{\mathrm{a}} \mathrm{Z}=[\mathrm{Fe} / \mathrm{H}]$.

${ }^{\mathrm{b}}$ Hot limit for all stars is $V-K=0.95$ unless otherwise noted.

possible, with observational errors dominating for metal-poor stars, for which the fractional uncertainty in the observed line strength is highest.

Quadratic behavior is seen again below in $\mathrm{Fe} 5270, \mathrm{Fe} 5335$, and $\mathrm{Na} \mathrm{D}$ and has important consequences for extracting gravities and metallicities from observed line strengths, as discussed below.

\subsection{Fe 5270 and $\mathrm{Fe} 5335$}

These features were the favored metallicity indices of Paper II and were used to estimate the abundances of M5, M71, and M67 in Paper III. They are good metallicity indicators because they show only a weak gravity dependence for giants and a modest trend with temperature. With the new, enlarged sam- 

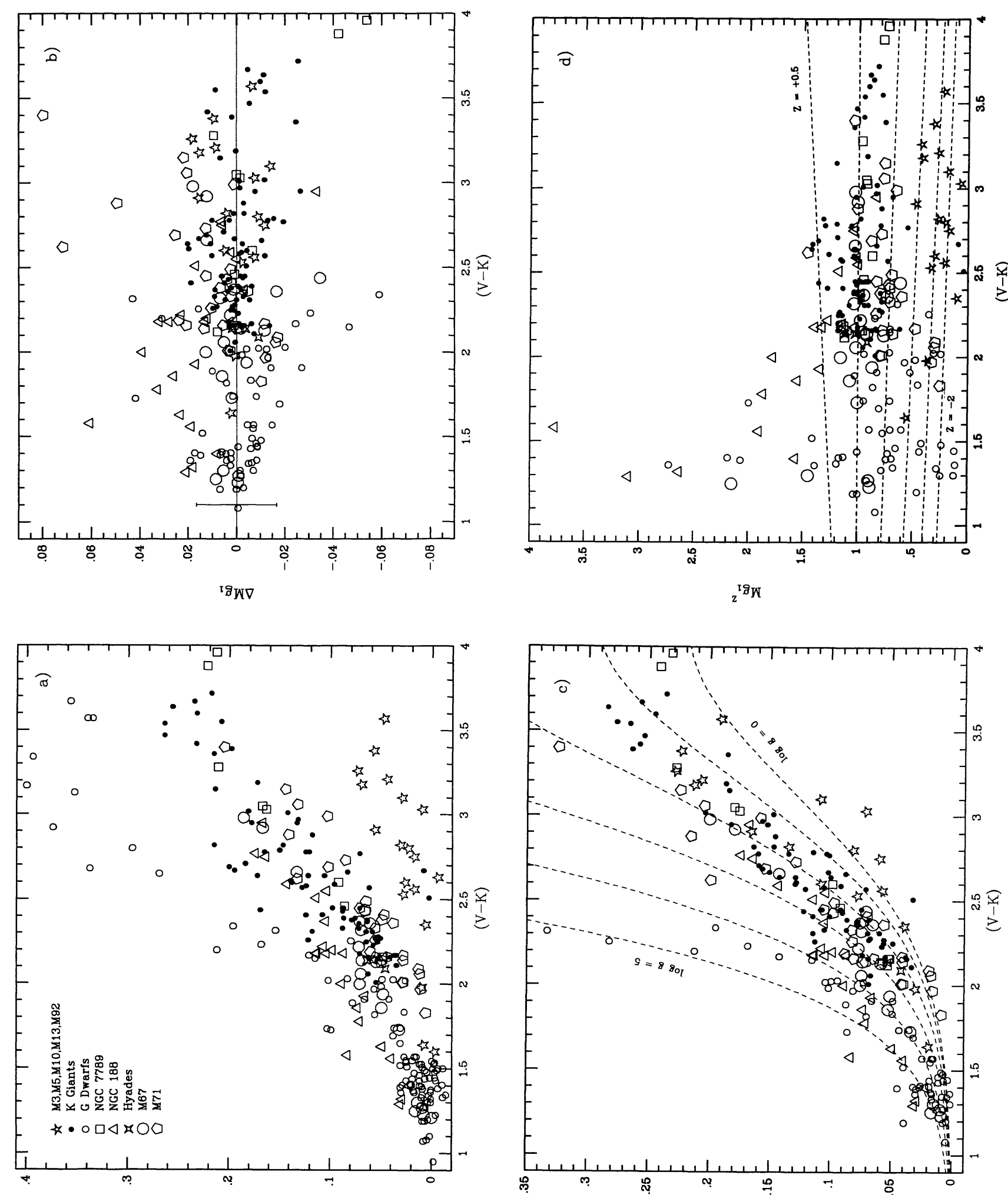

(Mex)

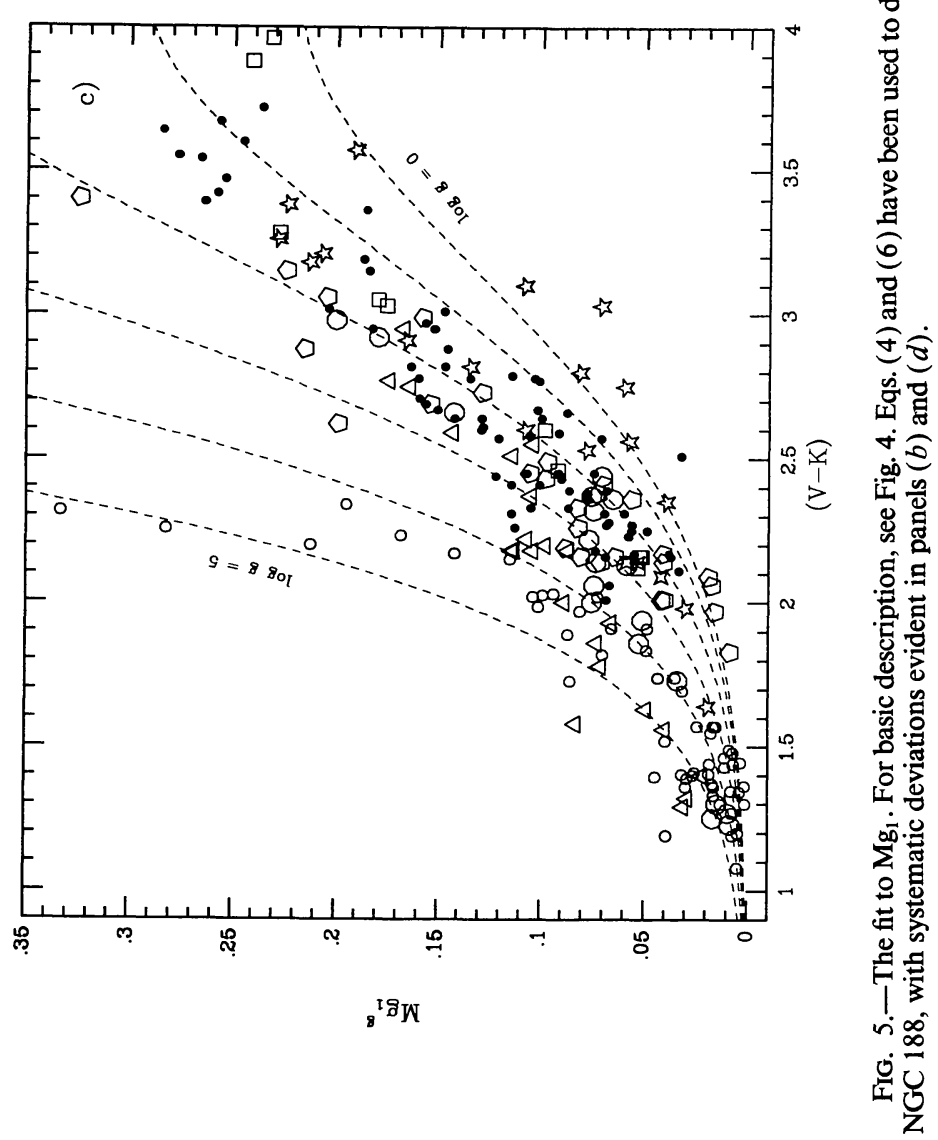




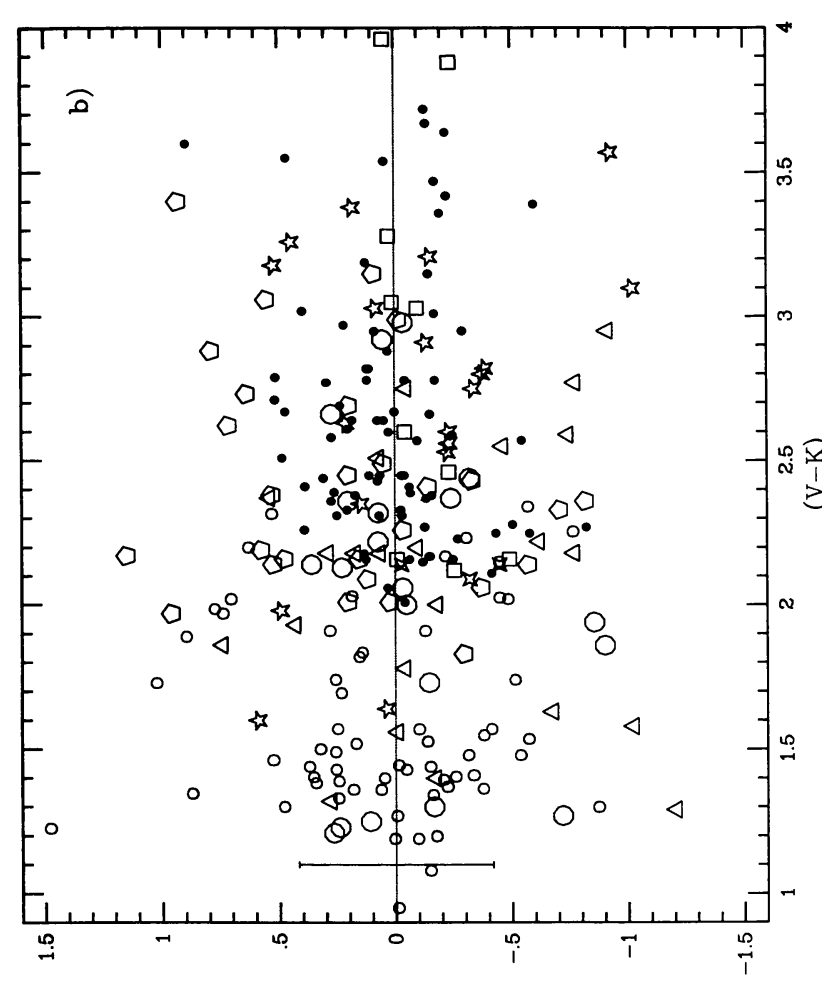

นLโร9จิNจ

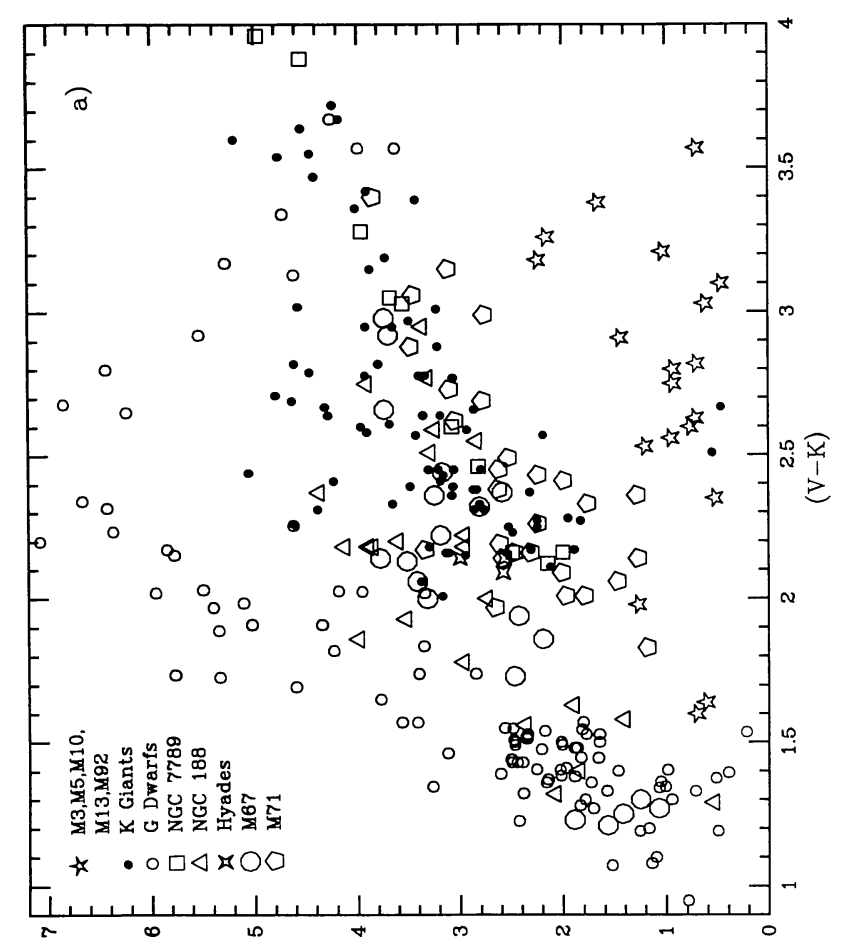

(Mex)LLโรq
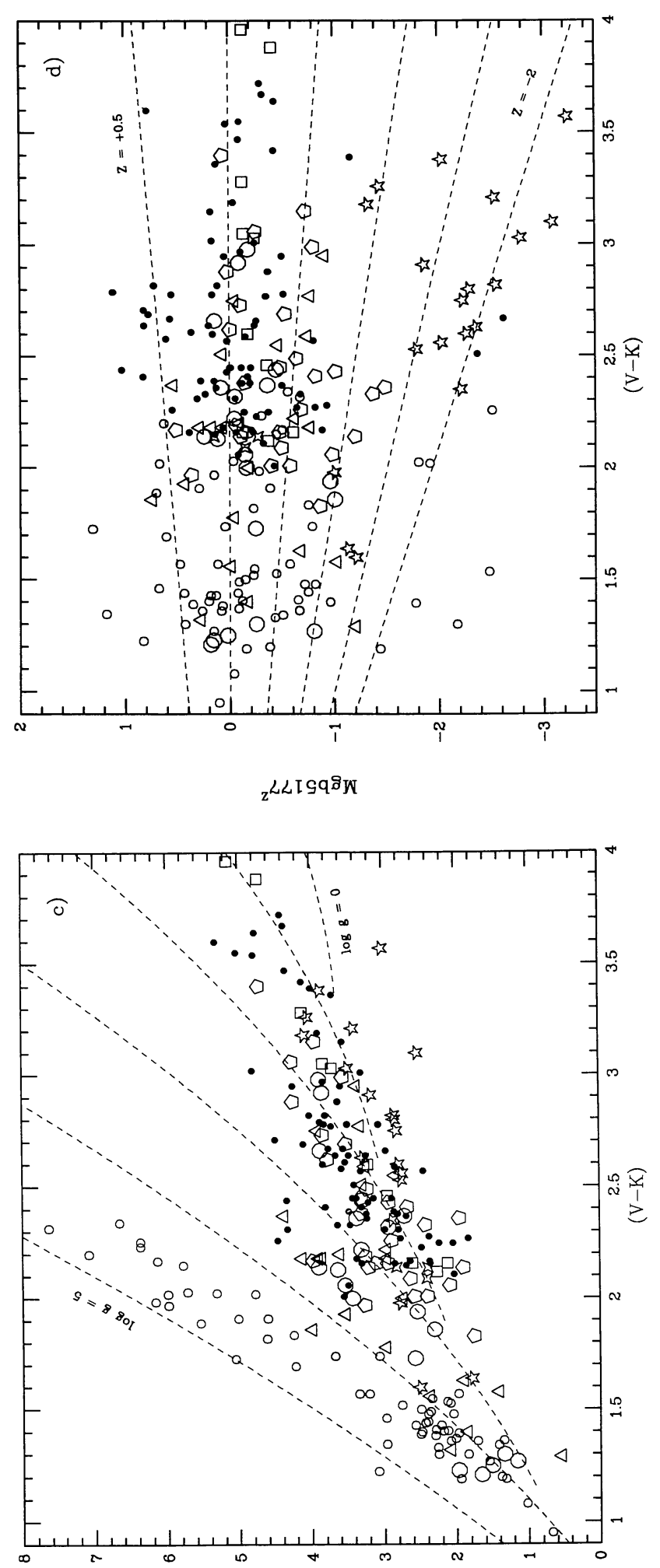

รLนโร98ิW 
ple, we can now see a slight divergence between the dwarf and giant-branch loci (see Figs. $7 a$ and $8 a$ ), but the spread on the giant branch itself is still small. The gravity dependence of $\mathrm{Fe}$ 5335 is slightly more marked than that of Fe 5270, and both features are quadratic with a weak rollover.

The fitting functions are shown in Figures 7 and 8. There do not appear to be any striking systematic residuals for any group of stars, and Table 1 shows that the rms residuals are only slightly larger than the observational errors.

\section{5. $\mathrm{Na} \mathrm{D}$}

$\mathrm{Na} \mathrm{D}$ is unique among the indices discussed here in being the only resonance line and thus the only feature affected by interstellar absorption. When we tried to fit the raw data without any correction for interstellar contamination, we saw that certain clusters seemed to be systematically offset. This could not be cured simply by altering the fitting function-the data themselves were inconsistent. In general, the clusters with the largest reddening showed the largest deviations, and these were positive, as would be expected for interstellar absorption.

The data of Cohen $(1973,1974,1975,1977)$ show that objects with moderate reddening in or near the Galactic plane show a mean interstellar Na D strength of about $0.3 \AA$ to $0.5 \AA$, but with large scatter. For lack of a better estimate, we applied a correction of $0.35 \AA$ to M67 and NGC 188 and $0.45 \AA$ to M71 and NGC 7789 based on their reddenings. From Cohen's work, it is also known that interstellar $\mathrm{Na} \mathrm{D}$ is not well correlated with reddening for high-latitude objects. Our clusters M3, M13, and M92 have been observed at high dispersion by R. P. Kraft with the Hamilton echelle spectrograph on the Shane telescope, and he kindly allowed us to inspect the interstellar components. We subtracted the following equivalent widths: $\mathrm{M} 3=0.8 \AA$; $\mathrm{M} 13=0.35 \AA$; $\mathrm{M} 92=0.1 \AA$. These are lower limits because any components near zero velocity would be obscured by strong $\mathrm{Na} \mathrm{D}$ emission in the night-sky spectrum of San Jose. An additional cluster M15 observed by Kraft showed very strong interstellar Na D in excess of $1 \AA$ despite its moderate reddening of only $0.12 \mathrm{mag}$, confirming the unpredictable nature of interstellar $\mathrm{Na} \mathrm{D}$ at high latitudes. For this reason we omitted clusters M10 and M5, for which we had no independent spectra. No correction was made to dwarf or field $\mathrm{K}$ giant indices. This is consistent with the fact that the highdispersion Arcturus and Procyon Atlases (Griffin 1968; Griffin \& Griffin 1979) show no measurable interstellar components.

After our analysis, Judith Cohen kindly called our attention to her previous high-dispersion measurements of interstellar Na D in M3, M13, M67, M71, and M92 (Cohen 1978, 1979, and 1980). Our corrections are within $0.2 \AA$ of her values for all clusters except M3, for which our correction is $0.8 \AA$ (based on Kraft's spectra), whereas Cohen found no detectable line. Our stars in M3 indeed have a mean residual of $-0.55 \AA$, indicating overcorrection. However, there are only two such stars, so the effect on the overall fit is negligible.

The resultant $\mathrm{Na} \mathrm{D}$ fit is shown in Figures $9 a-9 d$. It is fairly successful, but NGC 7789 shows a positive systematic residual of $\sim 0.3 \AA$, suggesting undercorrection. The rms fitting residual over all stars is $0.45 \AA$, compared to an observational error of $0.34 \AA$, a discrepancy that is no worse than for the other features. Because of the difficulties with the metal-poor cluster interstellar contamination, we consider the fit secure only above $[\mathrm{Fe} / \mathrm{H}]=-1.00$. The gravity function is quadratic and double-valued.

\section{6. $\mathrm{H} \beta$}

The behavior of the $\mathrm{H} \beta$ index is not yet fully understood. There is a moderate gravity effect, as evidenced by the separation in the raw data between the giants and the dwarfs (Fig. $10 a$ ). However, the giants among themselves are inconsistent. The field $\mathrm{K}$ giants and the metal-poor globular clusters lie highest, with M71 and NGC 7789 systematically offset to lower values. The amount of this shift, $\sim 0.35 \AA$, is larger than can reasonably be attributed to observational error. We have explored the possibility that $\mathrm{H} \beta$, like $\mathrm{H} \alpha$, might be contaminated by circumstellar emission, but the effect is several times too large (cf. Cacciari \& Freeman 1983). Metal-line contamination is inconsistent with the fact that the field $\mathrm{K}$ giants and the most metal-poor clusters agree. Again, cluster data deviate from field data in a manner for which we can offer no explanation.

In making a fit, we gave preference to fitting the field $\mathrm{K}$ giants in the belief that as a group they are the most representative of stars that contribute to the light in elliptical galaxies. We thus excluded NGC 7789 and NGC 188, although their residuals are shown in Figure $10 b$ and included in Table 1. Despite the problems, the rms residual turns out to be only $0.28 \AA$, which compares favorably to the observational error of $0.26 \AA$.

\section{7. $\mathrm{CN}$}

Our observations of $\mathrm{CN}$ lead to a rather different interpretation of this index compared to conventional views. Older opinion held that the $\mathrm{CN}$ difference between dwarfs and giants was due to surface gravity, whereas our evidence suggests that it is due mainly to mixing. $\mathrm{CN}$ is one of two indices whose behavior cannot be fitted by our assumed functional forms. The reasons why are apparent in the raw data in Figure $11 a$. It appears that the metallicity dependence is very different for giants and dwarfs-strong for the former, very weak for the latter. This breaks the assumption of separability for $p$ and $q$. Moreover, unlike other gravity-sensitive indices, there is a large gap between dwarfs and the cooler giants, with essentially no stars in between. This would require $p$ to be discontinuous between the two regimes. As a result, our methodology requires separate fitting functions for giants and dwarfs.

The two separate fits are shown in the iso-gravity contours of $p$ in Figure $11 c$. One line fits the whole dwarf sequence; the other fits the giants only. For dwarfs (including the turnoff stars and hot subgiants in M67 and NGC 188), we find no gravity dependence and very little dependence on $Z$. This is because there is essentially no $\mathrm{CN}$ in the spectrum; the behavior of the index reflects the continuum slope and the effect of $\mathrm{CH}$ and the Balmer lines in the sidebands. Two unusual dwarfs show abnormally strong $\mathrm{CN}$ (see below), but, apart from these, the rms scatter about the fit is consistent with observational error.

The functional form for the giants attempts to take into account the steep increase in $\mathrm{CN}$ with temperature along the giant branch. The fitted rise would perhaps be even steeper if 


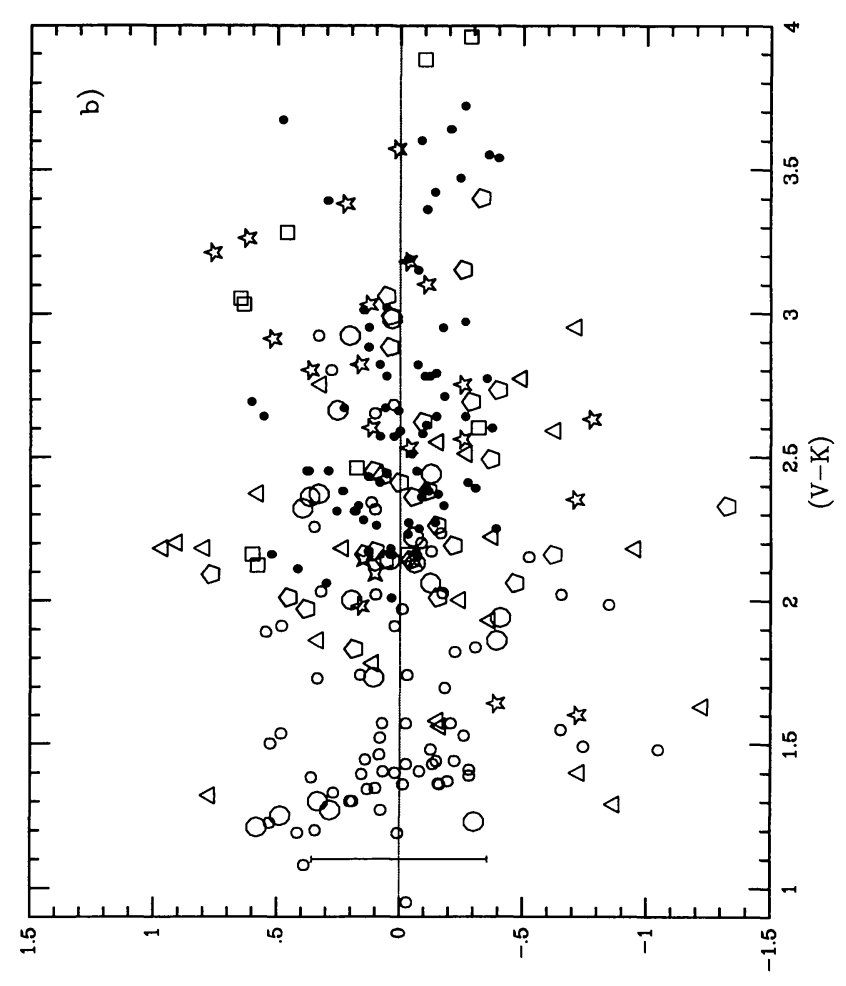

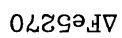

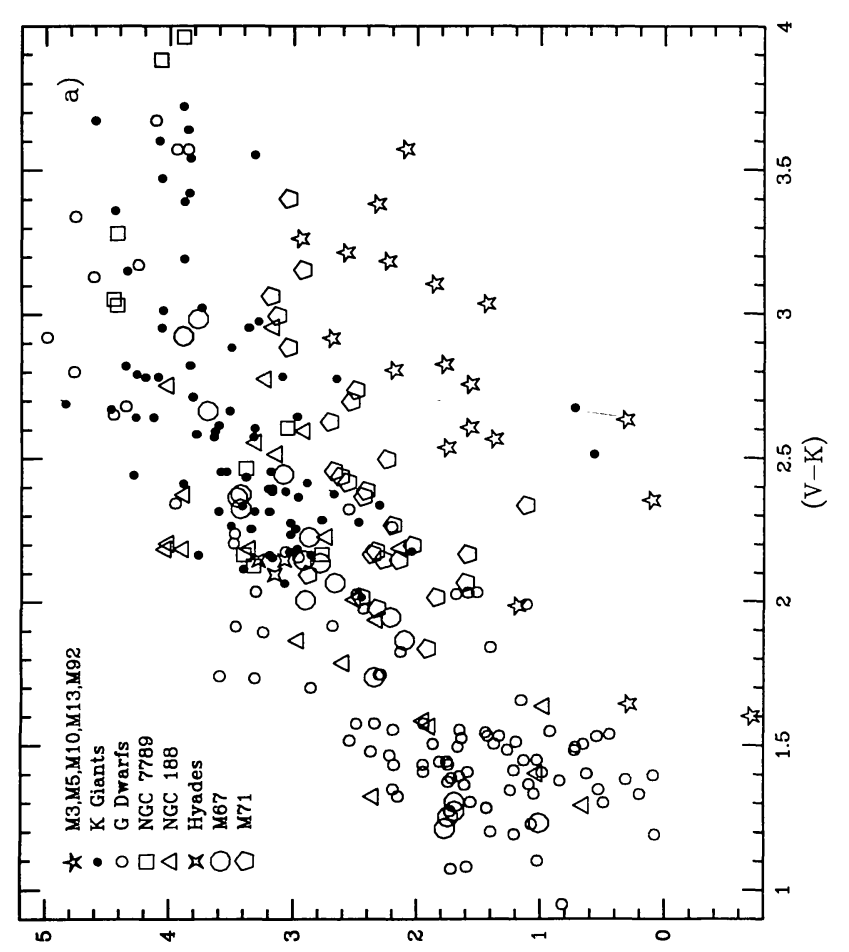

(MEI)OLZSaH

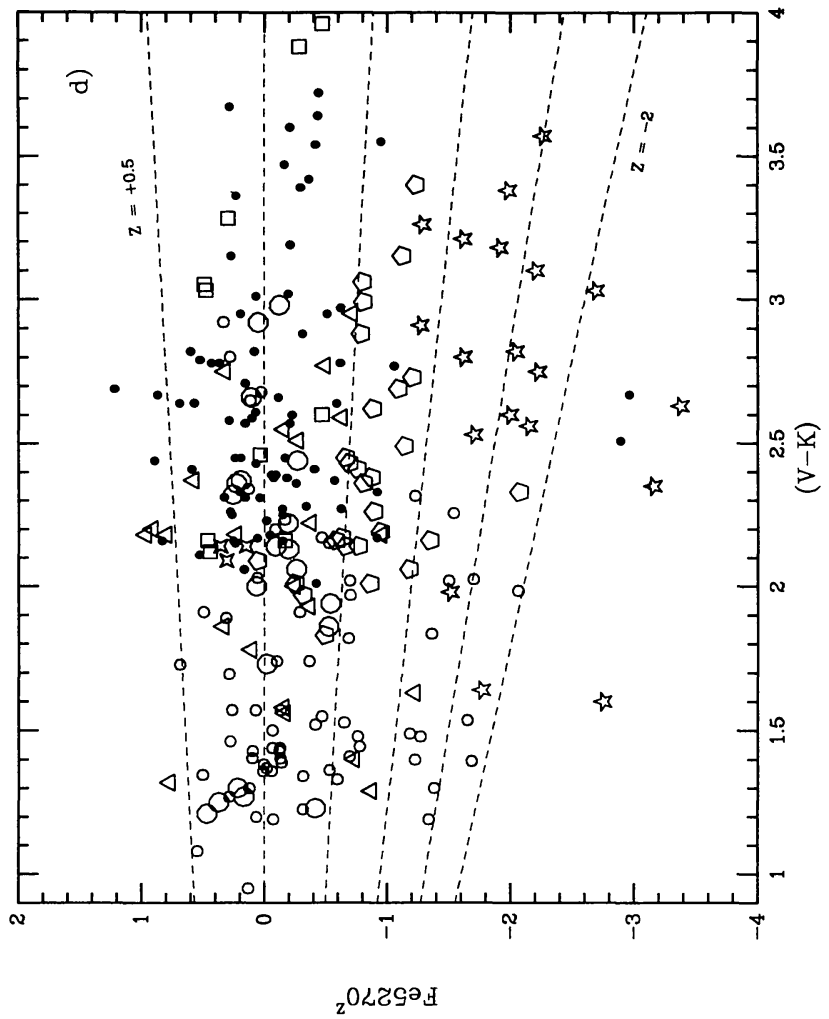

$\mathrm{z}^{02 Z \sigma_{\text {H }}}$

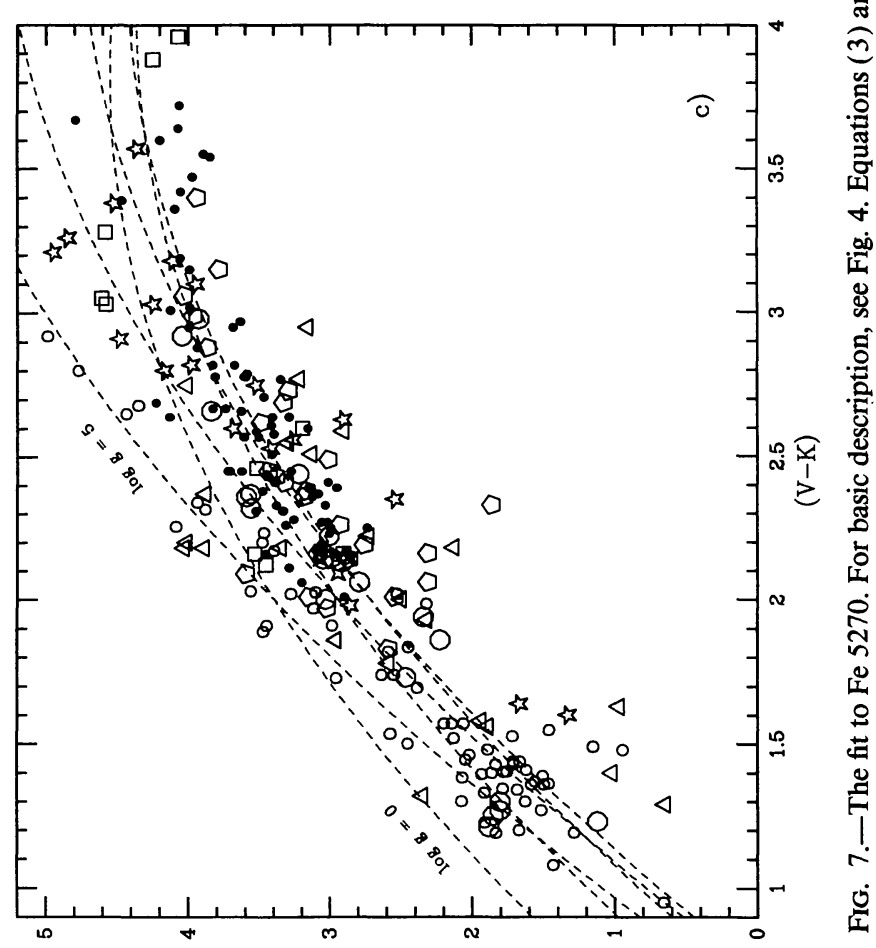

$802 Z 9 \partial^{4}$ 


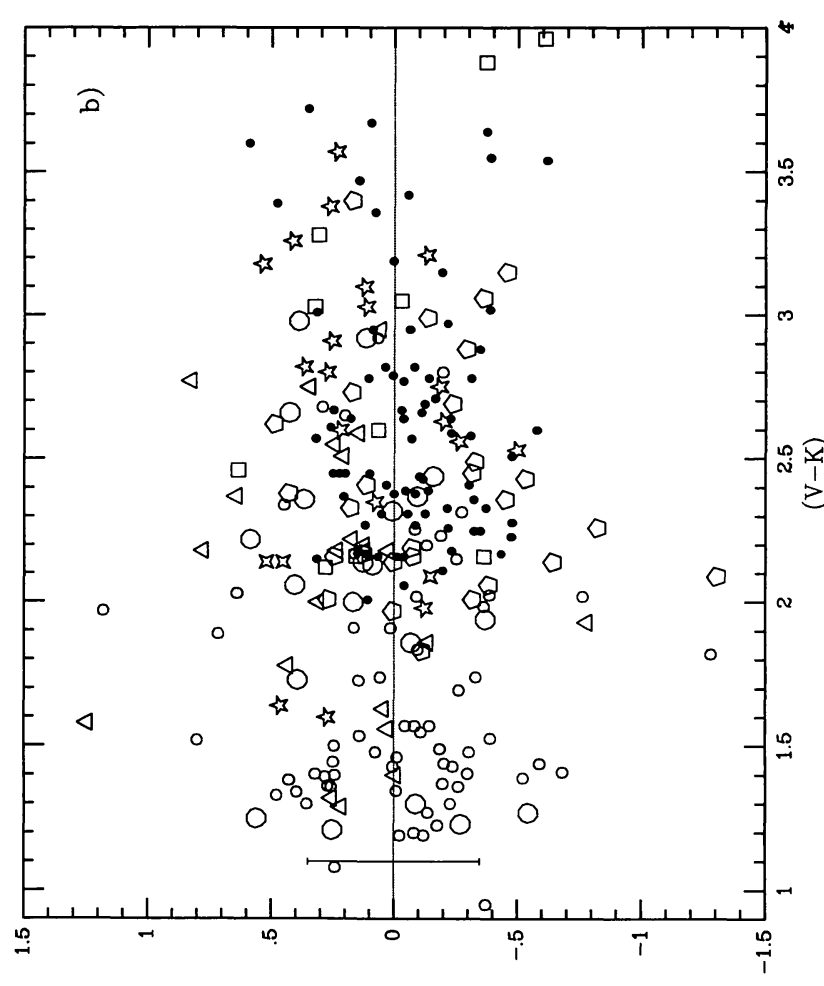

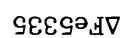

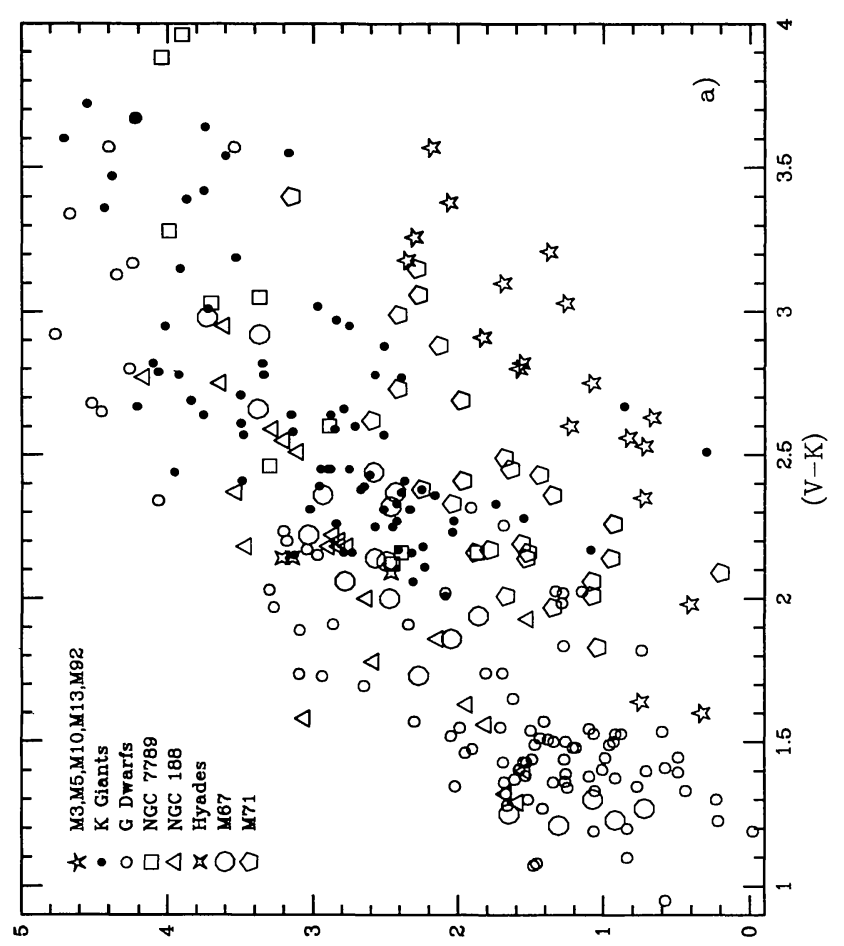

(мел)
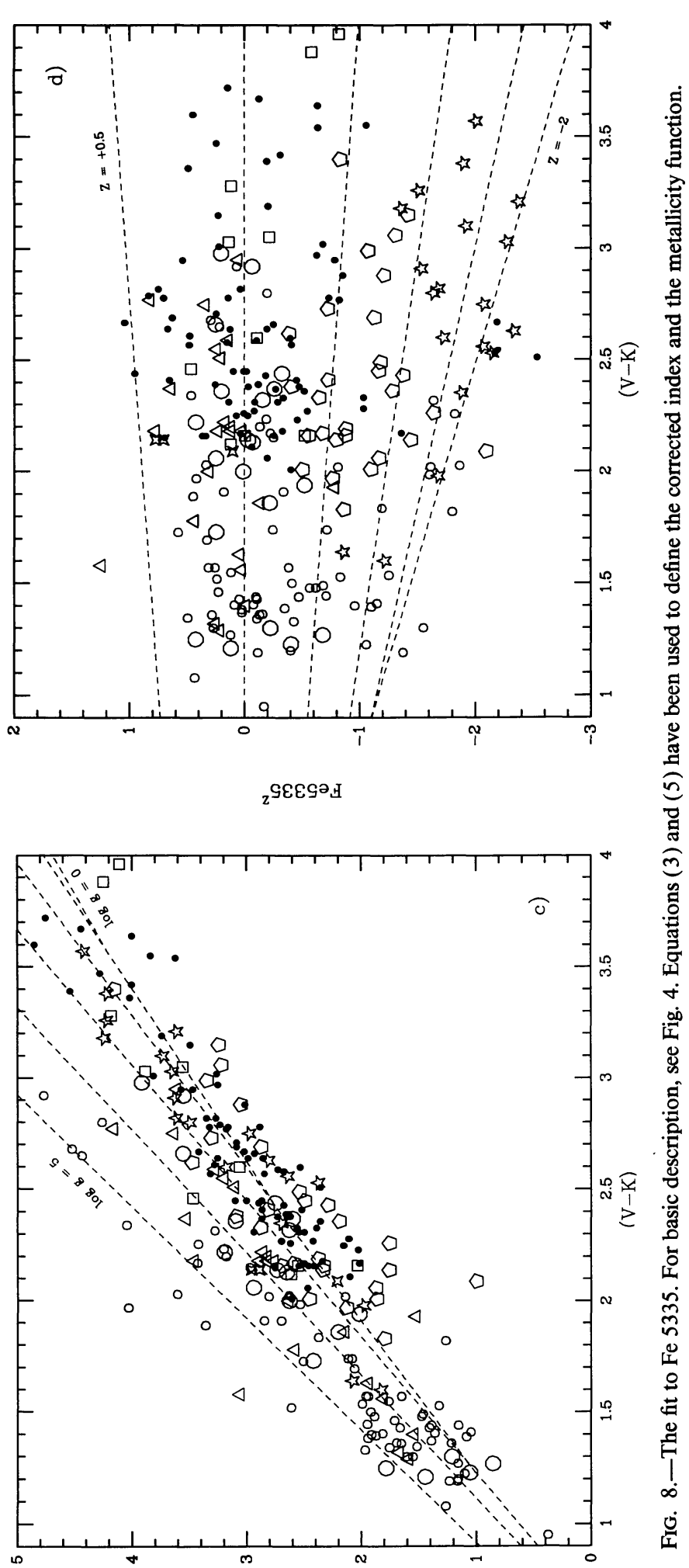

${ }_{\triangle}$ SEES 


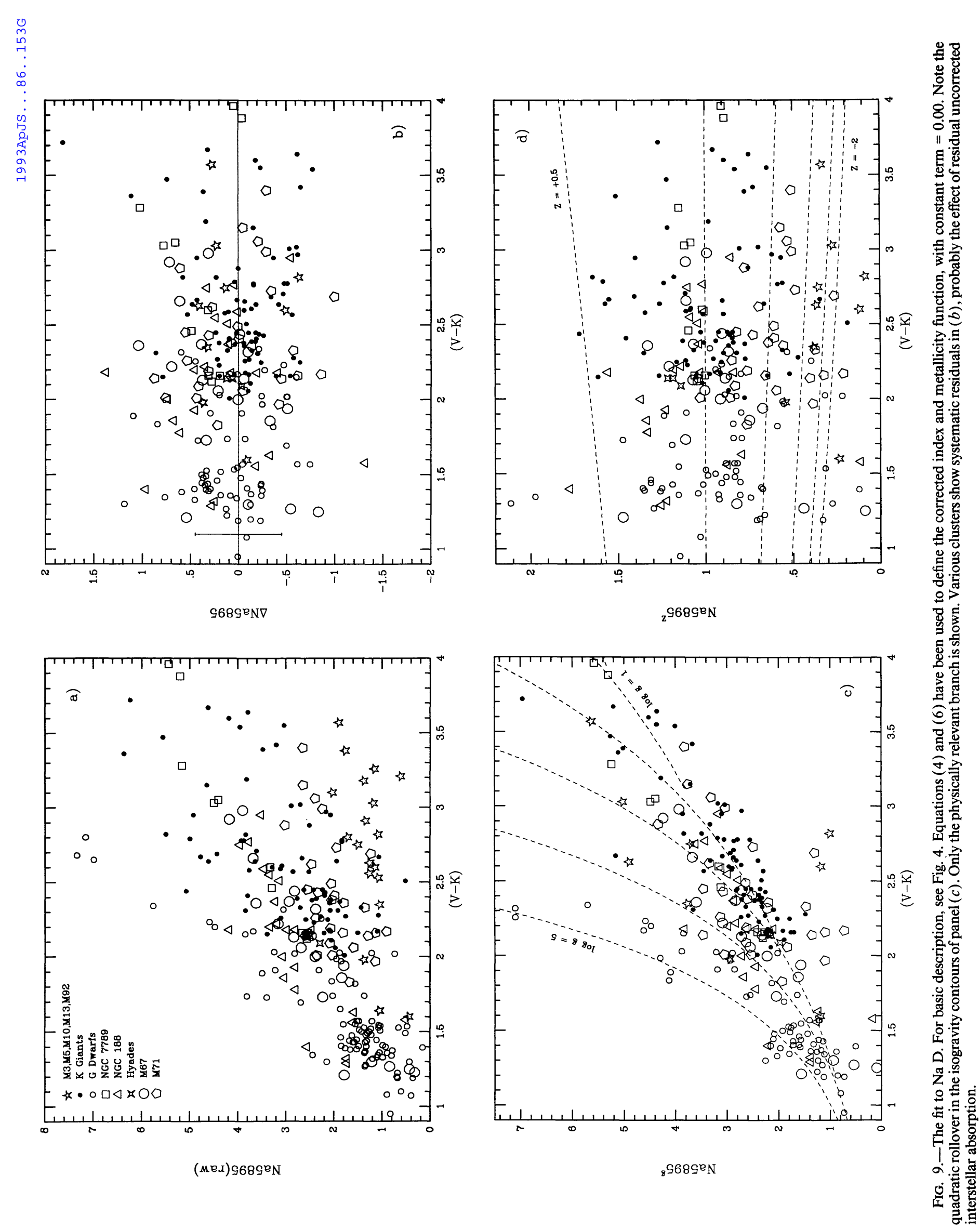




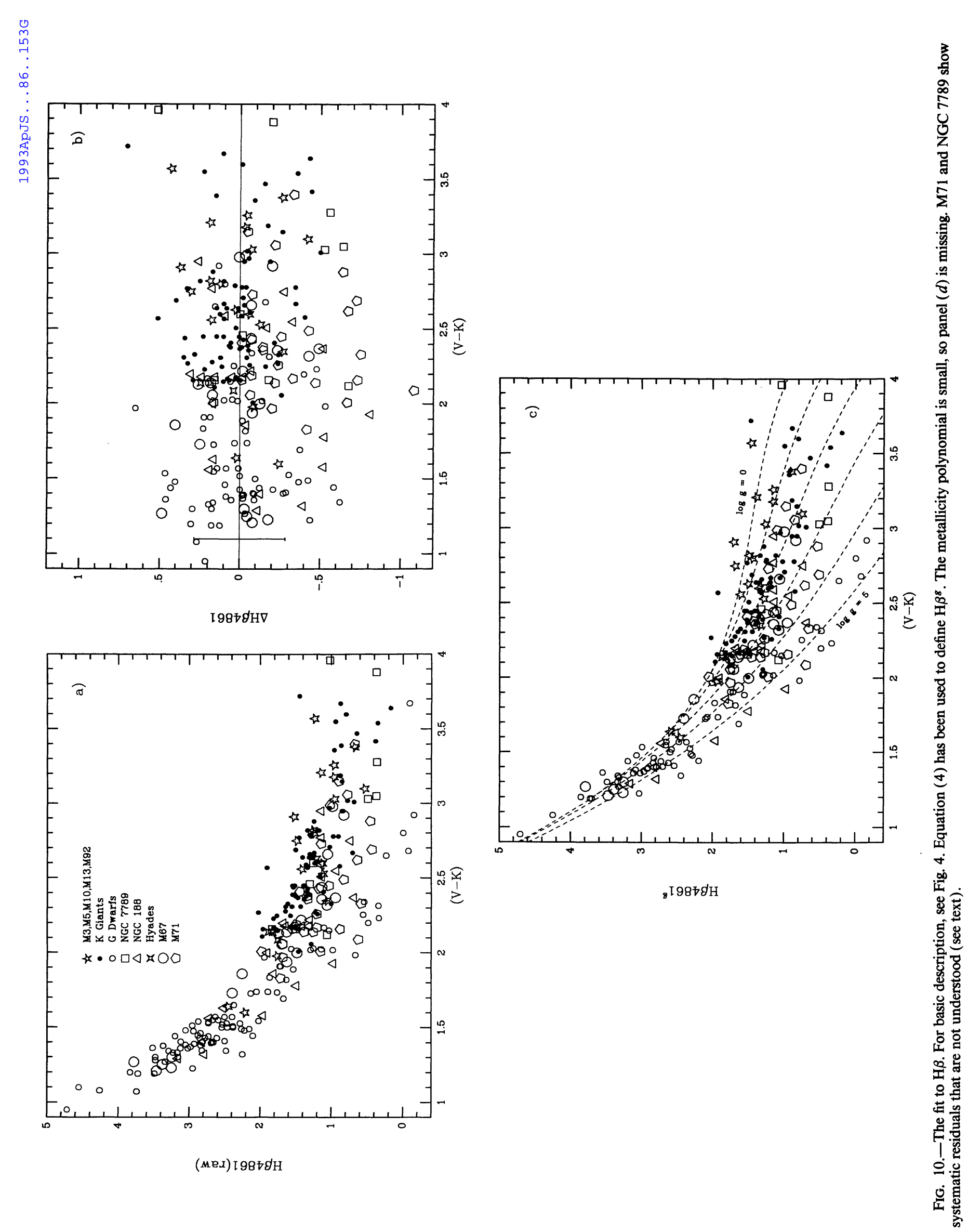




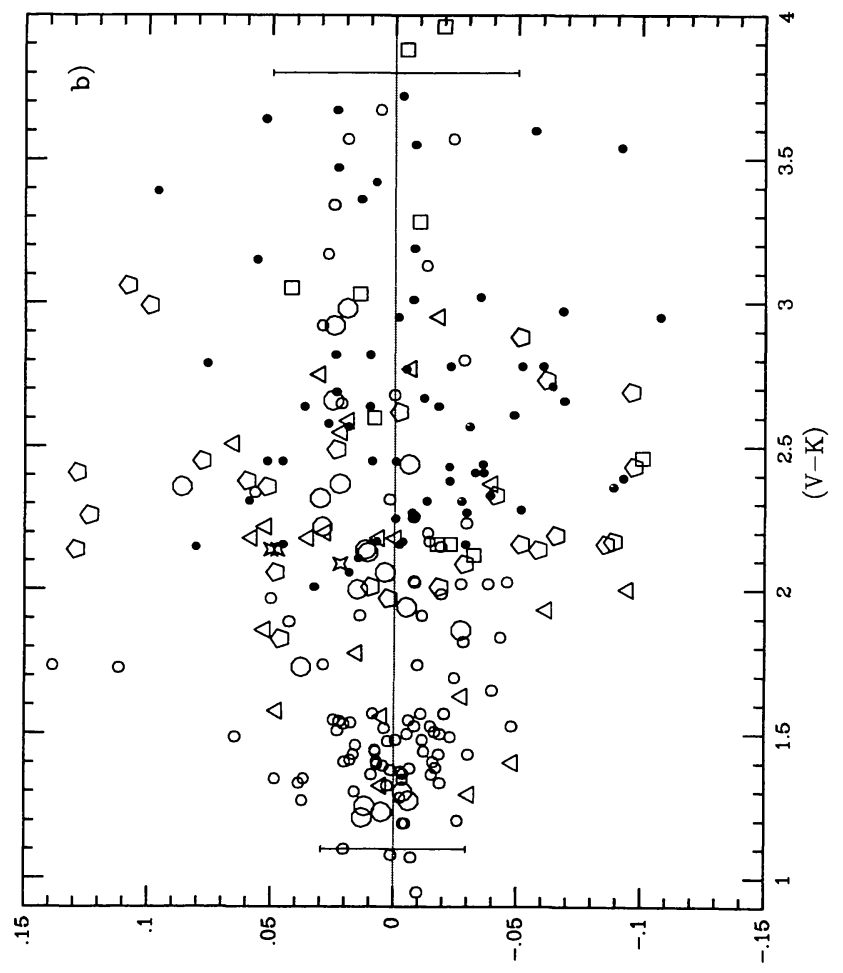

Nวจ

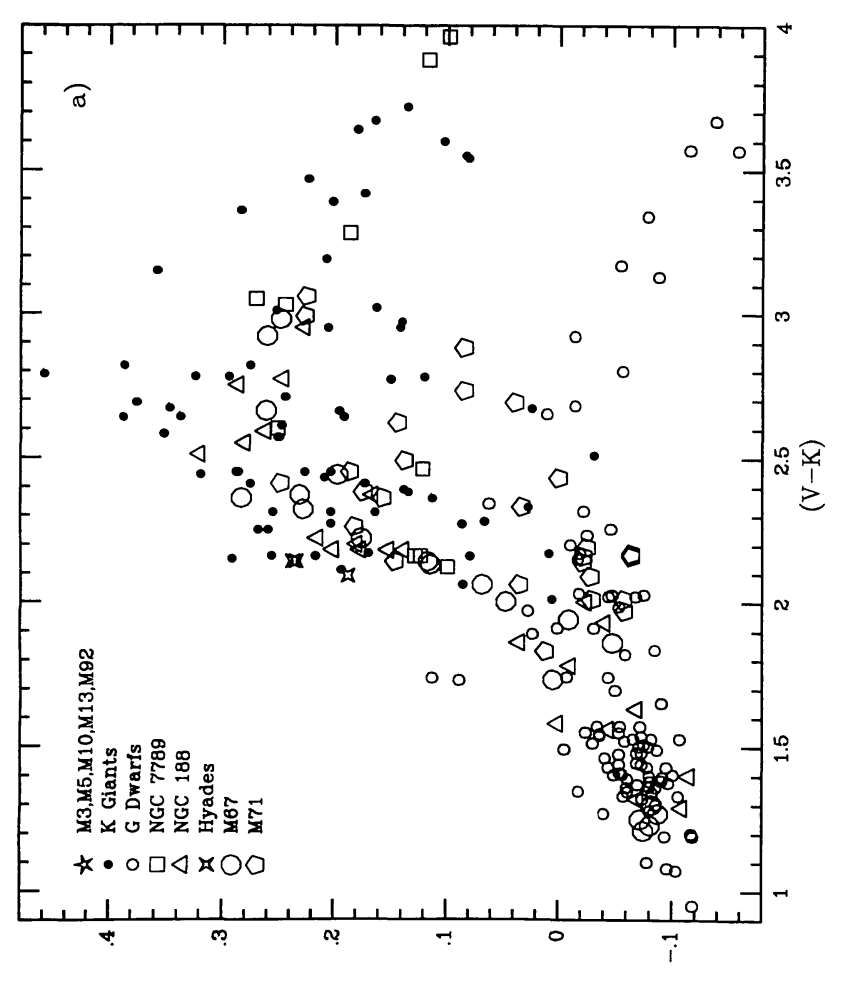

(Mеx)N

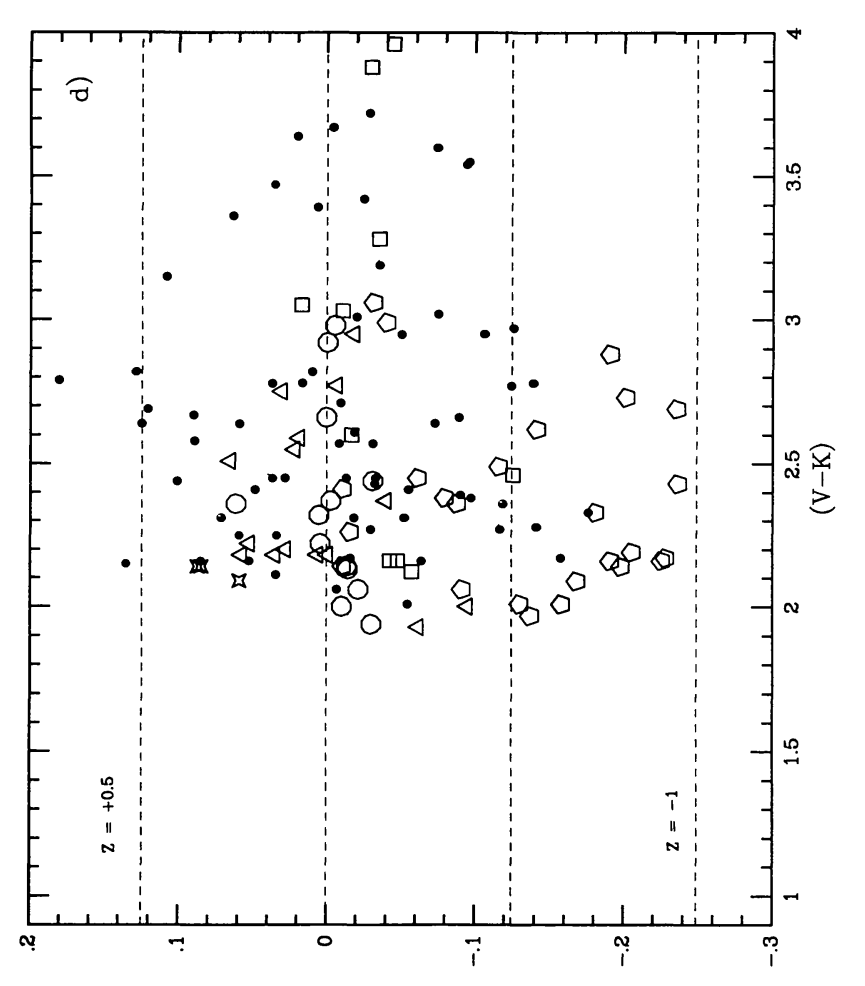

$z^{\mathrm{N}}$

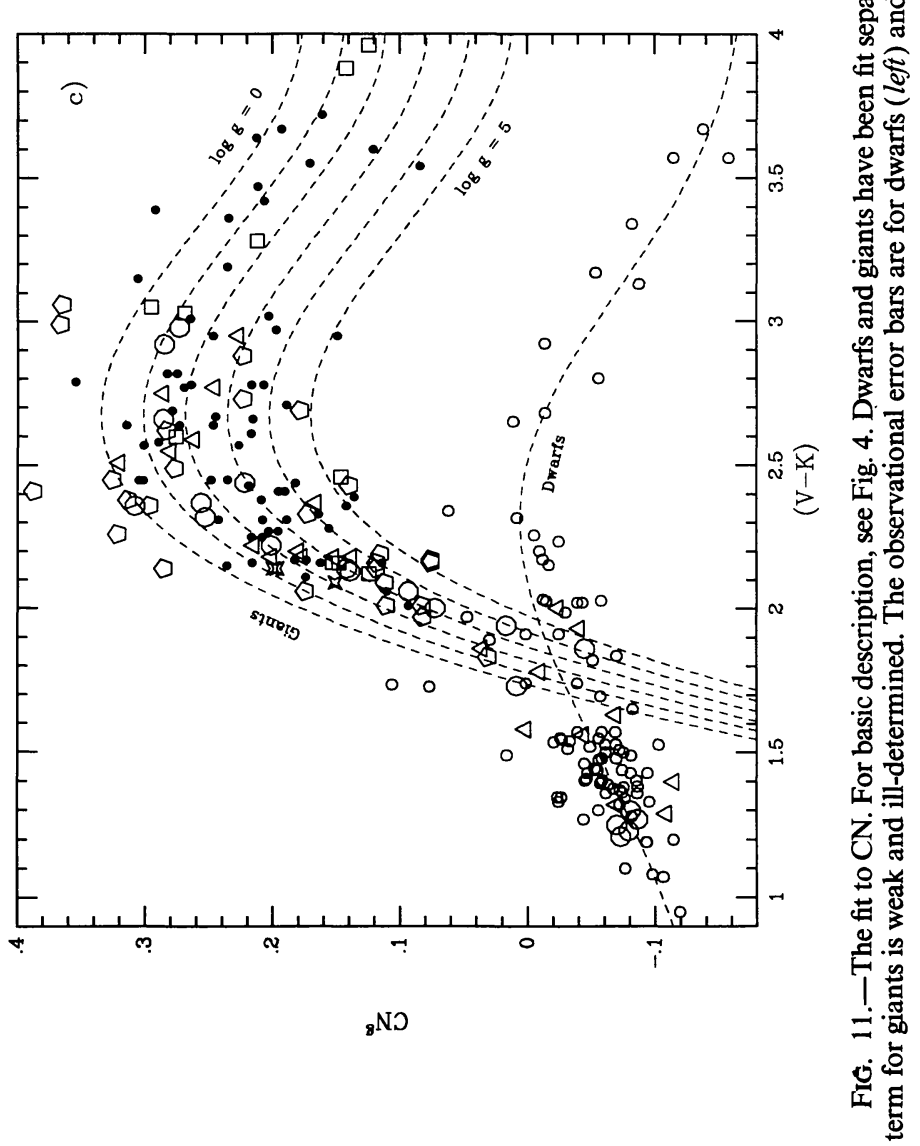


higher order terms in the fit had been allowed. The data are consistent with other CN surveys of K giants (e.g., Janes 1975; Hansen \& Kjaergaard 1971 ) which show a similar slope. Moreover, a recent high-dispersion study of M67 (Brown 1987) detects a sudden rise of $\mathrm{CN}$ on the lower giant branch and attributes it to the rapid onset of mixing. Translated to our color, this rise sets in at $V-K=2.10$ and is essentially complete by $V-K=2.20$. This is identical to the rise in M67 stars seen here. Further evidence for rapid mixing in M67 comes from the $C^{12} / C^{13}$ ratio (Gilroy \& Brown 1991), which begins to fall abruptly at the base of the giant branch as $\mathrm{CN}$ rises.

Our selection of giants goes well beyond M67, yet the same behavior is seen in all subsamples, including NGC 188, NGC 7789 , the Hyades, and the field stars. This suggests strongly that a sudden turn-on in mixing may be universal at the base of the giant branch, at least for metal-rich stars. ${ }^{8}$ In this picture, the discontinuity between dwarfs and giants is explained not by a strong gravity difference, the conventional view, but rather by the fact that the giants have mixed while the dwarfs have not. If so, this would make $\mathrm{CN}$ unique among our indices in that the fitting function does not model the behavior of the index at fixed atmospheric composition so much as evolutionary changes in that composition.

This conclusion is in excellent agreement with recent theoretical spectral syntheses of CN by Tripicco \& Bell (1990, 1991). These authors likewise concluded that the main influence on $\mathrm{CN}$ was mixing, not gravity, in $\mathrm{K}$ giants. As they point out, a metal-rich $\mathrm{CN}$ discontinuity of the sort seen here has important implications for population models in clusters galaxies, giving rise to a "guillotine effect" in which integrated $\mathrm{CN}$ values will depend critically on the number of stars on either side of this discontinuity. For this reason, it is important to understand the transition and its location in the H-R diagram as a function of age and metallicity.

We emphasize once again the relative in sensitivity of $\mathrm{CN}$ to gravity in this sample of stars, contrary to the classical view. For example, at fixed $V-K$, one can compare giants from M67 and NGC 7789 that have similar metallicities but gravities that differ by 1.3 dex (see Fig. 3). Figure 11 reveals no measurable difference in $\mathrm{CN}$, and the fitting function is consistent with this, returning only a weak and ill-determined gravity term (we have plotted formal isogravity contours in Fig. $11 \mathrm{c}$ up to $\log g=5$, but the real gravity effect is small and uncertain; these contours should not be taken literally). A significant effect due to gravity is still seen in the synthetic spectra of Tripicco \& Bell (1990, 1991). This contradiction might be explained by yet another evolutionary effect, namely the influence of age (and hence gravity) on degree of mixing. To match the data would require that younger, more massive stars mix less. Interestingly, such a trend has recently been reported by Gilroy et al. (1990) based on $C^{12} / C^{13}$ ratios.

Two strong-CN dwarfs, HD 75732 and HD 145675, are prominently visible above the dwarf locus in Figure $11 a$. Both have been noted in the literature previously (Greenstein \&

\footnotetext{
${ }^{8}$ The above reasoning applies only to metal-rich stars. We lack observations of $\mathrm{CN}$ for metal-poor stars owing to an error in the spectrograph setup, but metal-poor stars in any case show an intrinsic range in $\mathrm{CN}$ strength on the giant and subgiant branches and cannot be fit by any simple function such as used here (see review by Norris 1986).
}

Oinas 1974). One explanation for their high $\mathrm{CN}$ is that they are actually cooler subgiants, with $V-K \approx 2.10$ rather than $V-K \approx 1.75$, as we have them here. However, this would require an unusually large error in our $V-K, R-I$ temperature conversion, which typically shows scatter of only 0.15 mag. Such a cool temperature is also precluded by their high $\mathrm{H} \beta$ strengths (see Fig. 10 ). If the adopted $V-K$ 's are correct, these two stars stand out in every index and are by far the most metal-rich dwarfs in the sample. This might indicate either that interior mixing is common in very metal-rich dwarfs or that the metal abundance is so high that $\mathrm{CN}$ has become visible in the dwarf atmosphere. Either way, these interesting stars deserve further study.

Giant-branch mixing and/or high-CN dwarfs might contribute to the greatly enhanced $\mathrm{CN}$ that is visible in the integrated light of giant elliptical galaxies (Faber 1973), M31 globular clusters (Burstein et al. 1984; Tripicco 1989; Brodie, Huchra, \& Kent 1991), and even certain Galactic clusters (e.g., the detailed study of 47 Tuc by Tripicco \& Bell 1992).

\subsection{G-Band}

The G-band is the other index for which it has been necessary to break apart the fits for dwarfs and giants. The basic data in Figure $12 a$ show two loci that diverge in the vicinity of $V-$ $K=1.90$. For metal-rich giants cooler than this, there is essentially no dependence on $Z$, whereas metal-poor giants show generally depressed G-bands relative to metal-rich giants, but the behavior is erratic due to mixing (e.g., Carbon et al. 1982; Trefzger et al. 1983). Since the G-band evidently cannot be fitted by any one simple function in all giants, we have elected to delete the metal-poor giants and fit the remainder with no metallicity term. This single-line fit to the metal-rich giant locus is shown in Figure $12 c$.

For hot dwarfs, there is a metallicity trend in the expected sense that more metal-rich stars have stronger G-bands. For hot dwarfs below $V-K \leq 2.15$, the G-band is therefore a useful metallicity indicator. The metallicity dependence appears to weaken at cooler temperatures, but the exact behavior is not well established because the selection of stars with known metallicities among cool dwarfs is so poor. The present metallicity fit for dwarfs is not valid beyond about $V-K=$ 2.50, beyond which there are no data on dwarf metallicities.

The rms fitting residuals for both dwarfs and giants are consistent with observational error.

$$
\text { 4.9. } \mathrm{TiO}_{1} \text { and } \mathrm{TiO}_{2}
$$

The results for $\mathrm{TiO}_{1}$ and $\mathrm{TiO}_{2}$ are shown in Figure 13. When plotted versus $V-K$, neither index shows a difference between dwarfs and giants. The NGC 188 stars show a weak trend in their $\mathrm{TiO}_{1}$ residuals (but not $\mathrm{TiO}_{2}$ ) in the same sense as seen previously for $\mathrm{Mg}_{1}$ and $\mathrm{Mg}_{2}$. A metallicity effect is evident for the metal-poor stars. To save space, we have overplotted the functions $\mathrm{TiO}^{g}$ (which contain only terms in $V-K$ ) on top of the raw data, with the result that the metal-poor stars lie below the solar loci (dashed lines). However, when metallicity is taken into account, they obey the fitting functions well, and their residuals about the full fit are normal. The residuals for all stars are generally comparable to the observational error, although the scatter in $\mathrm{TiO}_{1}$ appears to be slightly high in M71. 


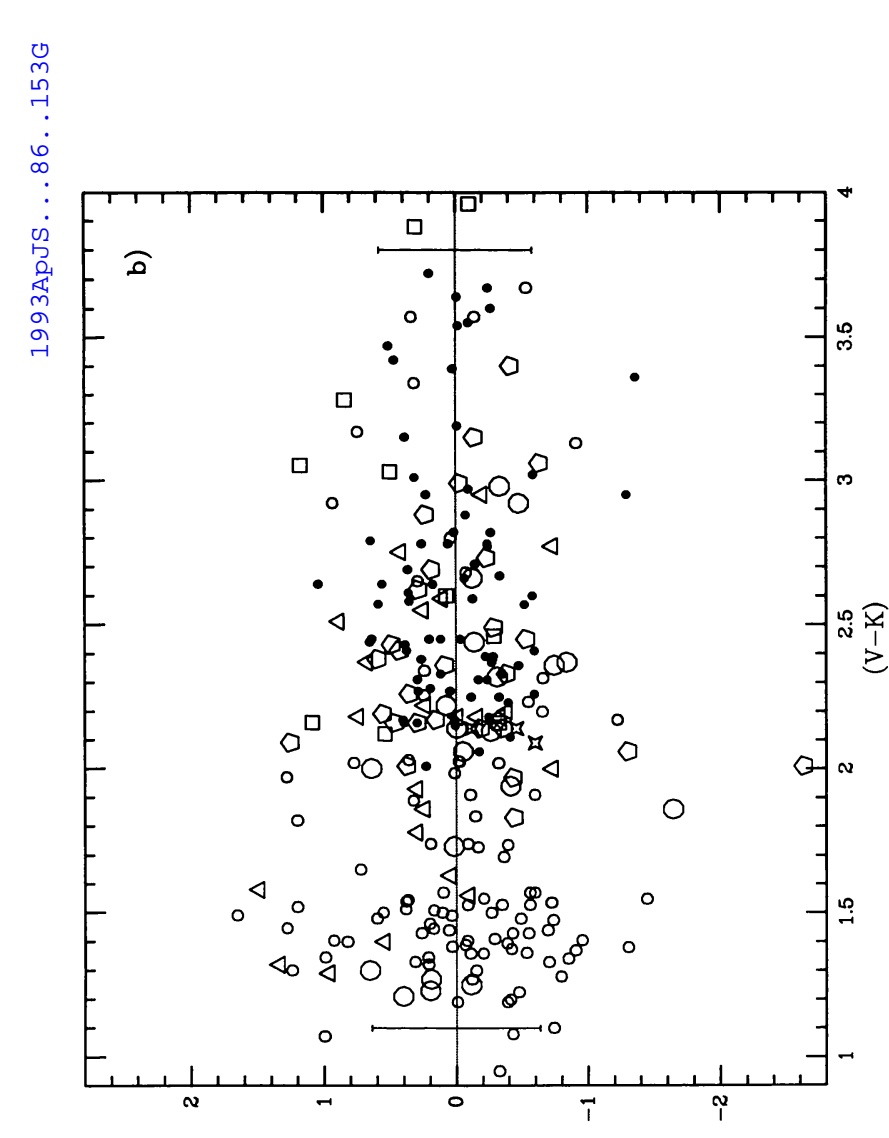

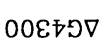

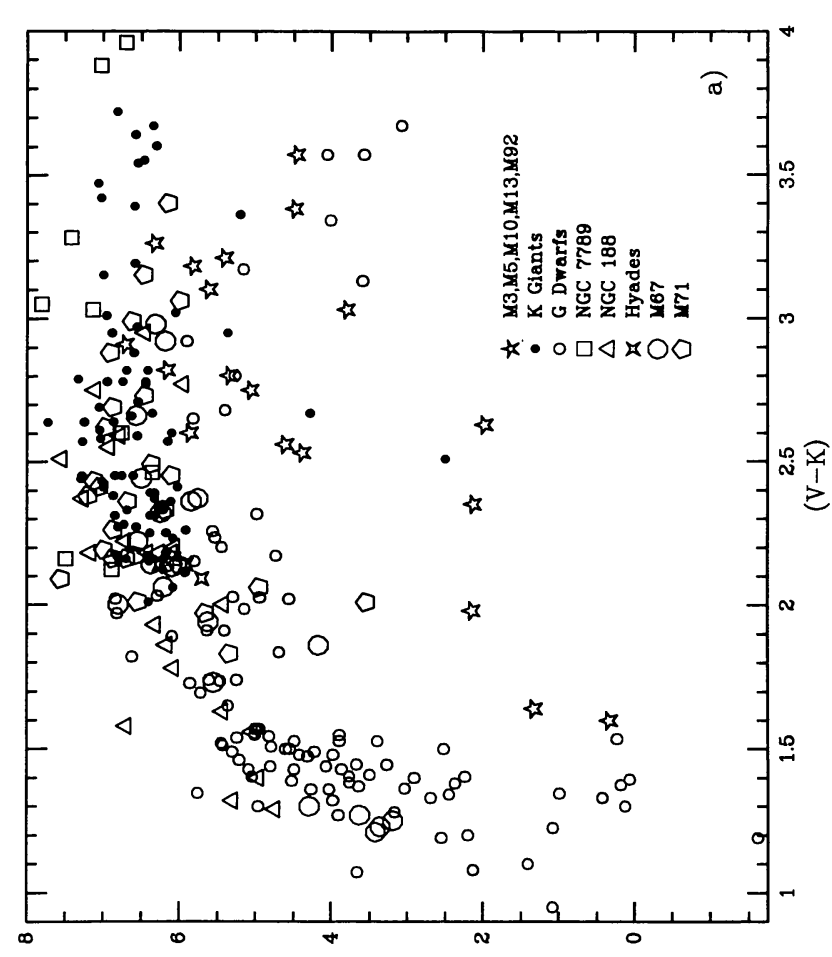

(Mеx)00EтD
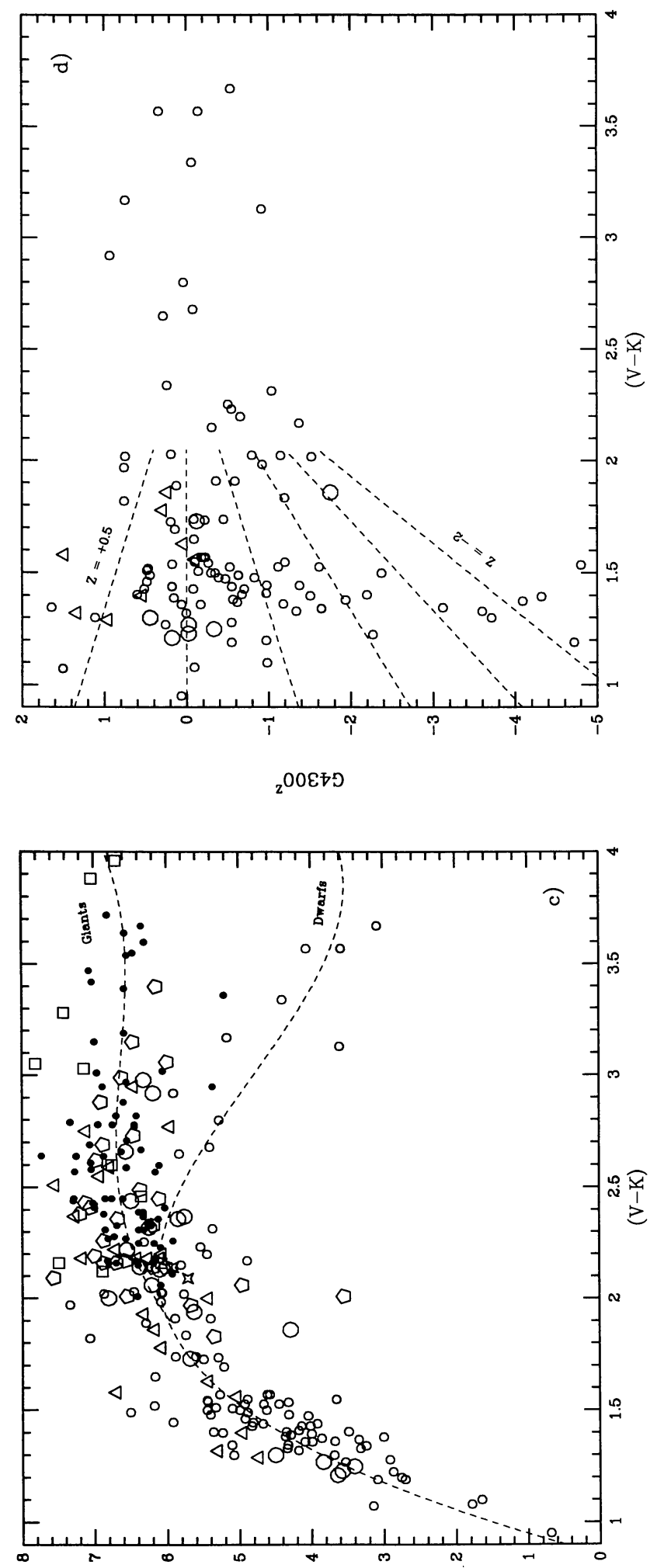

๑00Еฤつ 

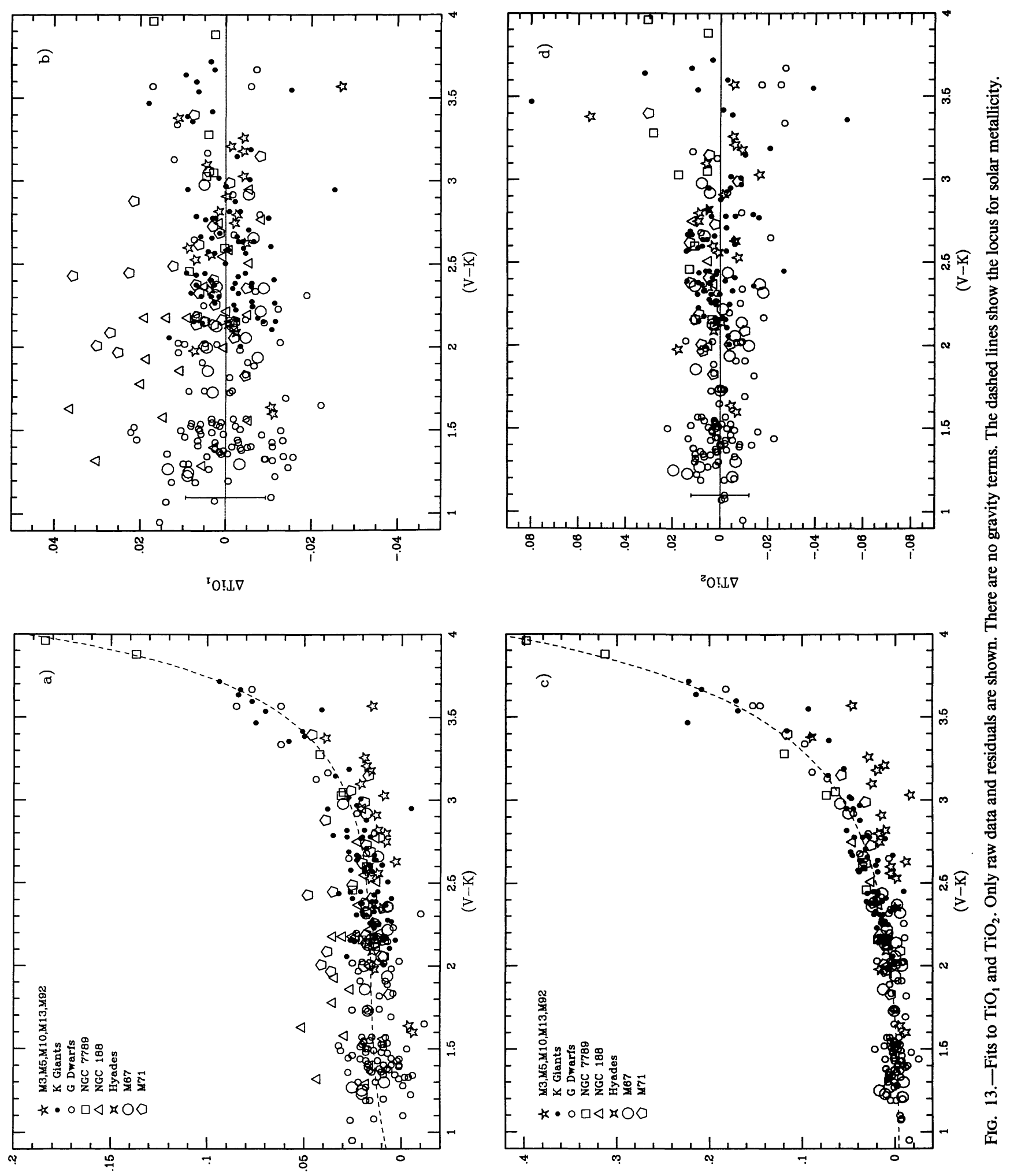

(MEI) $)^{\text {TotL }}$

(MEI) $)^{z}$ ILL 


\section{INVERTING THE FITTING FUNCTIONS: OUTPUT VALUES $\mathrm{OF}[\mathrm{Fe} / \mathrm{H}] \mathrm{AND} \log g$}

\subsection{Deriving Output Parameters}

The fitting functions can be turned around to deduce output metallicities and gravities for individual stars. This process tests the accuracy of the functions by showing that, on average, the output parameters agree with the input parameters and that the different line indices yield consistent results (with the few exceptions noted previously). We also explore how accurately basic stellar parameters can be estimated from the present spectra. In the process, we derive new abundances for all clusters and for many of the stars with no previous abundance estimates.

For each star we first take $V-K$ and $\log g$ as given and deduce an output value of $[\mathrm{Fe} / \mathrm{H}]$ from each of the six best features that are most sensitive to metallicity. Then we alter the process, take input $[\mathrm{Fe} / \mathrm{H}]$ as given, and deduce an output log $g$ from the two best features that are most sensitive to gravity. The results are shown in Table 5, which compares output and input parameters in each feature for all modeled stars (not all stars can be modeled since some lack basic input data or lie outside the region of validity of the fits). The estimated output parameters from the various features are averaged together (see the Notes to Table 5 for the weights) to form a final estimated abundance, $[\mathrm{Fe} / \mathrm{H}]_{\text {out }}$, and a final output gravity, $(\log g)_{\text {out }}$.

The output gravity and metallicity estimates from certain lines pose a special problem because of the rollover (quadratic term) in the gravity or metallicity functions. Stars in the double-valued region above the rollover are easily handled by taking the proper root, but stars below the rollover have no solution (imaginary roots). This is not unnatural, as most such stars have simply been scattered there by observational errors. Rather than concoct "guesstimates" for these stars, we have elected to omit them. However, as a consequence, the plots of the output versus input quantities for these indices tend to look biased, owing to the preferential loss of stars with negative observational errors. This does not mean that the original fits to the indices themselves are biased, as can be seen from the symmetric distribution of the line-strength residuals in Figures 4-13. The problem is worse for the less sensitive indices, as they are more perturbed by observational errors. It is also worse for weaker lined stars as, again, observational errors are fractionally more important. These effects can be seen in the following diagrams.

Input and output values of $[\mathrm{Fe} / \mathrm{H}]$ and $\log g$ are illustrated for seven features in Figures $14 a-14 g$ and for four features in Figures $15 a-15 d$. Stars with no solution (imaginary roots) are indicated at the bottom of each figure. Certain groups of stars are deleted from certain diagrams (i.e., no dwarfs in $\mathrm{CN}$, no giants in $\mathrm{CH}$ ) because data are missing, the spectral feature is insensitive to that parameter in those stars, or because some stars lie outside the region of validity of the fits.

Aside from the illusory bias due to imaginary roots, the output parameters for all features appear to be nicely distributed about the input lines, indicating at most small systematic errors in the fitting functions. (The only exception might be a slight overestimate of $[\mathrm{Fe} / \mathrm{H}]_{\text {out }}$ in $\mathrm{Fe} 5270$ by $\sim 0.1$ dex for the highest metallicity stars.) Most of the few discrepant points can be explained by small errors in the assumed $V-K$ 's.

The small scatter of the field $\mathrm{K}$ giants ( solid dots) in the $\mathrm{Mg}_{1}$ and $\mathrm{Mg}_{2}$ diagrams is misleading. This scatter is anomalously small owing to the force-fit of gravity to $\mathrm{Mg}_{2}$ for these stars; it has no physical significance.

\subsection{Output Metallicities}

The output metallicities derived by inverting the fitting functions are important in two respects. First, it is a requirement that the gross properties of the output plots, such as mean slope and curvature, be consistent with the $45^{\circ}$ line. Agreement here shows that we have not made major errors in either the first or the second derivatives in the fitting functions. Beyond that, the output metallicities give new, semi-independent metallicity estimates for each star and cluster- "semi-independent" because, although any one object contributes somewhat to the original fit, its fractional influence, or even that of a whole cluster, is rather small. This is especially true for the metallicity fits, for which the total number of input data is large. The output metallicities therefore effectively rank-order each object relative to all the others, and it is highly significant that the output rank-ordering agrees closely with the input rank-ordering. The output metallicities are in essence new lowresolution estimates, with the rest of the data set providing the calibration. As such, they constitute significant new determinations.

Table 7 summarizes the mean residuals in $[\mathrm{Fe} / \mathrm{H}]_{\text {out }}$ by stellar group for field stars and for clusters separately. For each cluster, the mean $[\mathrm{Fe} / \mathrm{H}]_{\text {out }}$ by feature is given for the eight features that are at all sensitive to metal abundance. The most reliable features have been averaged to yield a final weighted $[\mathrm{Fe} / \mathrm{H}]_{\text {out }}$ for each cluster and the net difference, $\Delta[\mathrm{Fe} / \mathrm{H}]$, from the assumed input cluster metallicity.

For the five metal-rich clusters, individual feature estimates agree within the errors (including NGC 188, despite the observed systematics), and the weighted averages agree well with the input values within the expected errors. This indicates satisfactory consistency in the fits and no measurable systematic errors in the overall abundance calibrations. For metal-poor clusters, the agreement is slightly poorer but is everywhere better than 0.2 dex, which is roughly the expected error. We conclude that the cluster metallicities are well understood.

For the field giants and dwarfs (Table 7B), we give the rms $\Delta \mathrm{Fe}$ for each feature and $\Delta \mathrm{Fe}$ averaged over all features. Again the results are consistent with the input metallicity scale within a few hundredths of a dex. We must repeat, however, the caveats about the metallicity scale for field $\mathrm{K}$ giants above solar metallicity that we wrote in Paper II (Faber et al. 1985). This portion of the scale is determined by the so-called super-metalrich (SMR) stars (cf. Spinrad \& Taylor 1969). Since high-dispersion studies of these stars are still discrepant, we have to resort to input metallicities from various intermediate-band photometric studies (Gottlieb \& Bell 1971; Gustafsson et al. 1974; and Hansen \& Kjaergaard 1971). We have presented arguments elsewhere (Paper II and Bond et al. 1985) as to why we think these stars really are above solar in metallicity. However, the present output metallicity estimates clearly do not 

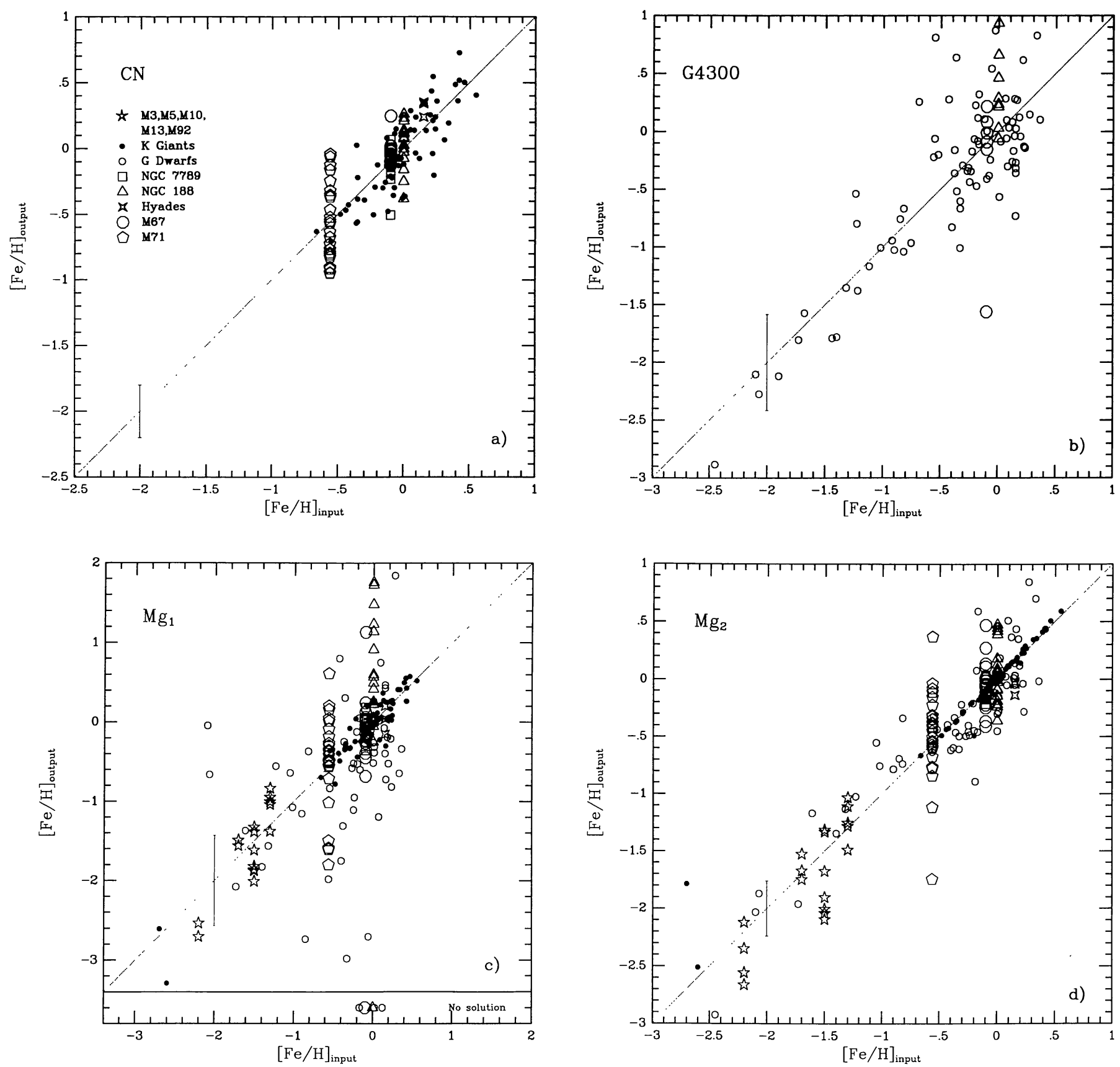

FIG. 14.-Output values $[\mathrm{Fe} / \mathrm{H}]_{\text {out }}$ vs. input values $[\mathrm{Fe} / \mathrm{H}]_{\text {in }}$ for seven metallicity-sensitive features. Error bars are the average of the dwarf and giant errors as tabulated in Table 8. Output values should lie along the one-to-one line if a star's index is perfectly fit. Missing stars signify either missing data or that the feature is not metallicity sensitive for that star. The small residuals for field $\mathrm{K}$ giants in the $\mathrm{Mg}$ indices are spurious, due to the gravity fit to $\mathrm{Mg}_{2}$ (

Stars that yield imaginary roots (for the quadratic $Z$ functions) are labeled "No Solution" and are plotted at bottom. Output distributions look asymmetric about the one-to-one line in some panels owing to loss of these stars, but feature residuals are symmetric, indicating the basic fits are unbiased (see text). Fits appear to be satisfactory for all features except $\mathrm{Fe} 5270$, for which $[\mathrm{Fe} / \mathrm{H}]_{\text {out }}$ may be 0.1 dex too high for the most metal-rich stars.

provide any independent information, since they are simply forced to be consistent in the mean with the assumed input scale.

To take the analysis one step further, we note that a single input metallicity was assumed for each star. The observed agreement among $[\mathrm{Fe} / \mathrm{H}]_{\text {out }}$ for different indices therefore requires that the relative abundances of these elements must all be moving together in lockstep. This agrees with the body of data that is steadily accumulating on element/Fe ratios in disk and halo stars (Lambert 1987; Gehren 1988; Wheeler et al. 1989), which suggests a nearly universal nucleosynthesis enrichment "trajectory" for elements in our Galaxy. While most of our data support this hypothesis, the $\mathrm{CN}$ index illustrates the fact that evolutionary processes may also play a role in deter- 

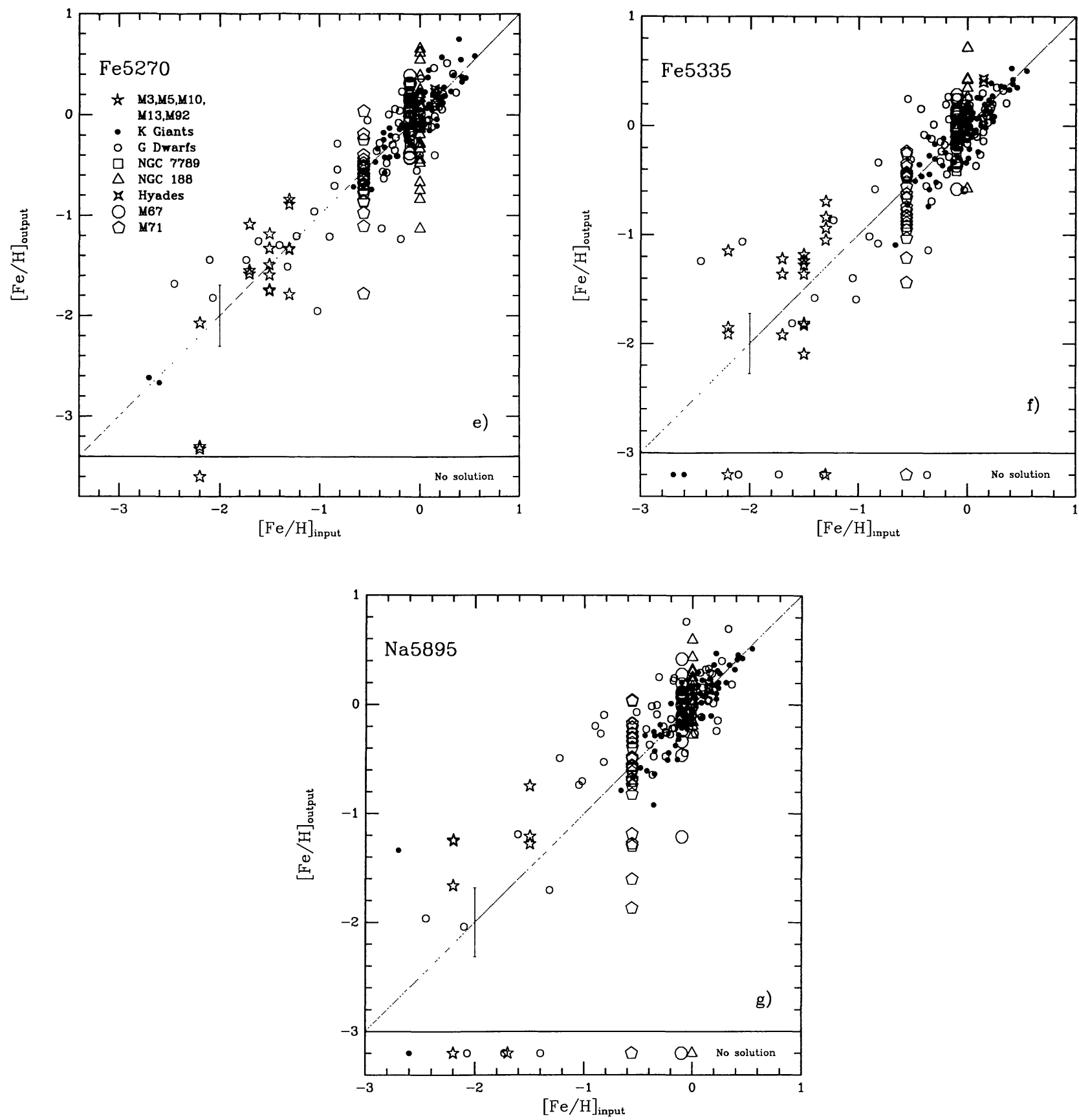

FIG. 14-Continued

mining at least some line strengths. Our method of using fitting functions permits direct tests of whether the solar neighborhood abundance trajectories can be used to model self-consistently the integrated spectra of elliptical galaxies, external globular clusters, and stars in the bulge of our own Galaxy. Initial results (Worthey et al. 1992) suggest that indeed the $\mathrm{Mg} / \mathrm{Fe}$ abundance ratio differs in giant ellipticals compared to the metal-rich calibrating stars here.

To assess the present spectra as abundance indicators, we calculate the rms error per star in $[\mathrm{Fe} / \mathrm{H}]_{\text {out }}$ as deduced from each feature. These errors have been calculated from the residuals in Figure 14 and are tabulated in Table 8. No error has been allowed for in $[\mathrm{Fe} / \mathrm{H}]_{\text {in }}$, so these errors are upper limits. The values given are means over the cluster and field stars together and represent the error for an average-quality observation in the present sample. The relative sizes of these errors in the various features have been used to fix the feature weights for computing the mean $[\mathrm{Fe} / \mathrm{H}]_{\text {out }}$ for each star in Table 5 , as given in the notes there. Finally, using these weights, we can predict what the net error of the mean should be if all feature 

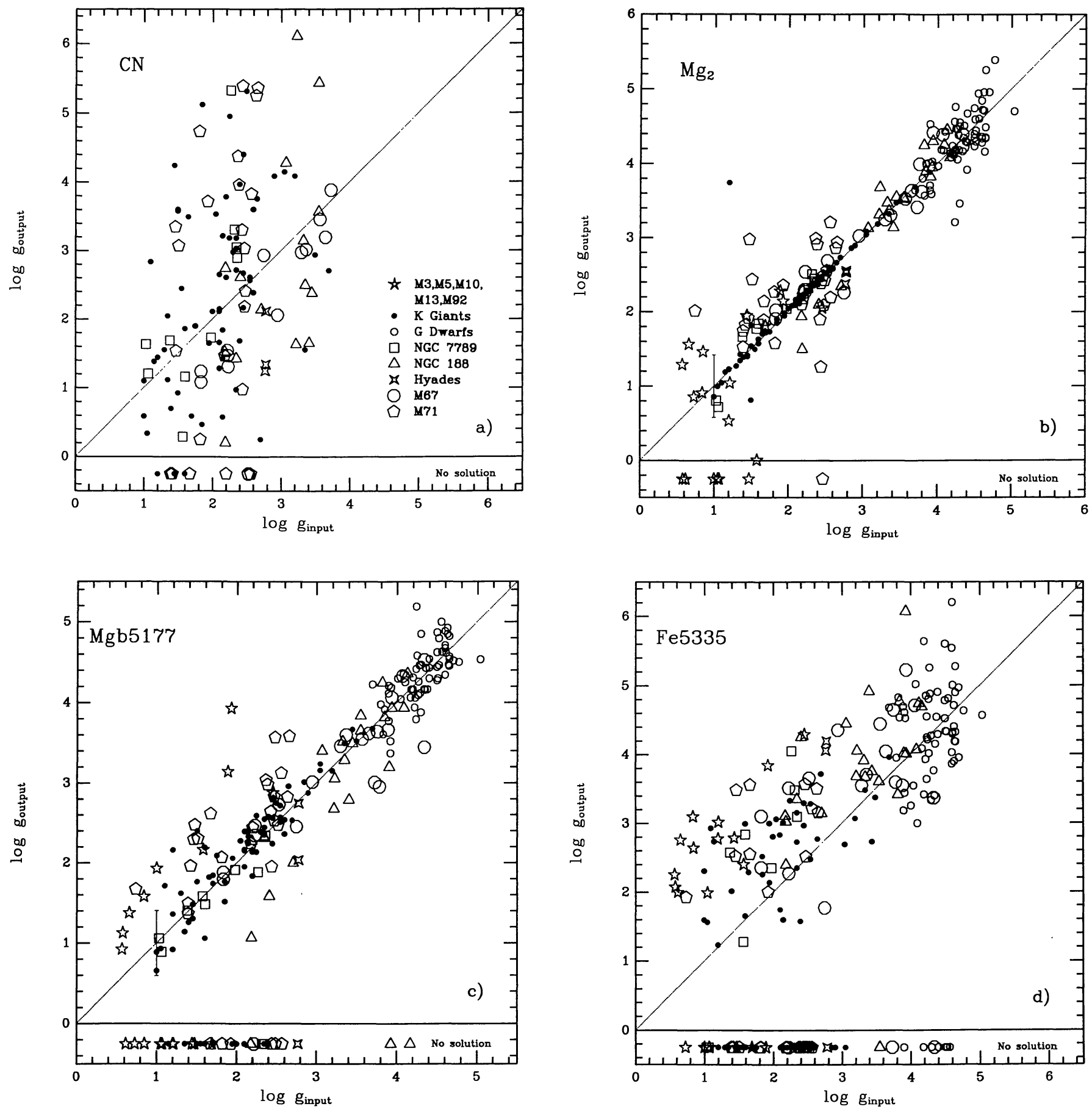

FIG. 15.-Same as Fig. 14, showing $\log g_{\text {out }}$ for four spectral features. Imaginary roots (No Solution) are more frequent in this figure, owing to the stronger quadratic dependence of the gravity fits. Note the relative unimportance of gravity for $\mathrm{CN}$ among giants, compared to the strong metallicity dependence shown in Fig. 14a. Error bars are as tabulated in Table 9. The small residuals for field $\mathrm{K}$ giants in the $\mathrm{Mg}$ indices are again spurious, due to the gravity fit to $\mathrm{Mg}_{2}$ (see text). No error bar is calculated for Fe 5335 since the distribution is too highly skewed. The log $g_{\text {out }}$ diagram for Na D is not shown but shows a scatter similar to but somewhat greater than Fe 5335.

errors add in a Gaussian manner. This is called the net predicted error and is also given in Table 8 .

For both dwarfs and giants, five features turn out to give useful estimates of $[\mathrm{Fe} / \mathrm{H}]$. The net predicted error in $[\mathrm{Fe} /$ $\mathrm{H}]_{\text {out }}$ based on a weighted mean of those five features is 0.10 dex for giants and 0.135 dex for dwarfs. The actual observed differences $[\mathrm{Fe} / \mathrm{H}]_{\text {out }}-[\mathrm{Fe} / \mathrm{H}]_{\text {in }}$ in Table 5 are 0.16 dex and 0.21 dex, respectively. These values are marginally consistent if errors of roughly $0.1 \mathrm{dex}$ in $[\mathrm{Fe} / \mathrm{H}]_{\text {in }}$ are allowed for. Overall, we conclude that $[\mathrm{Fe} / \mathrm{H}]$ can be deduced from a single spec- 
TABLE 7

MeAn Derived [Fe/H $]_{\text {out }}^{a}$ FOr Groups of Stars, by Feature

\begin{tabular}{|c|c|c|c|c|c|c|c|c|c|c|c|}
\hline Group & $\mathrm{CN}$ & $G$ band & $\mathbf{M g}_{2}$ & $\mathbf{M g}_{1}$ & $\mathrm{Mg} b$ & Fe52 & Fe53 & $\mathrm{Na} D$ & {$[\mathrm{Fe} / \mathrm{H}]_{\text {out }}^{\mathbf{a}}$} & {$[\mathrm{Fe} / \mathrm{H}]_{0}$} & $\Delta \mathrm{Fe}^{b}$ \\
\hline \multicolumn{12}{|c|}{ a) Clusters } \\
\hline NGC 188 & $+0.021^{c}$ & $+0.345^{d}$ & +0.043 & +0.307 & -0.221 & -0.108 & +0.127 & +0.104 & +0.039 & +0.000 & +0.039 \\
\hline NGC 7789 & -0.171 & $\ldots$ & -0.052 & -0.107 & -0.203 & -0.050 & -0.082 & +0.032 & -0.091 & -0.100 & +0.009 \\
\hline M 67 & $-0.200^{c}$ & $+0.00 \sigma^{d}$ & -0.056 & -0.102 & -0.190 & -0.122 & -0.047 & -0.078 & -0.088 & -0.100 & +0.012 \\
\hline Hyades & +0.318 & $\ldots e$ & -0.070 & +0.033 & -0.081 & +0.196 & +0.297 & +0.168 & +0.123 & +0.150 & -0.027 \\
\hline M 71 & -0.554 & $\ldots e^{e}$ & -0.481 & -0.490 & -0.411 & -0.639 & -0.690 & -0.614 & -0.535 & -0.560 & +0.025 \\
\hline M 3 & $\ldots f$ & $\ldots e^{e}$ & -1.647 & -1.514 & -1.928 & -1.407 & -1.501 & . & -1.599 & -1.700 & +0.101 \\
\hline M 5 & $\ldots f$ & $\ldots e^{e}$ & -1.232 & -1.041 & -1.211 & -1.235 & -0.878 & $\ldots$ & -1.119 & -1.300 & +0.181 \\
\hline M 10 & $\ldots f$ & $\ldots e$ & -1.780 & -1.721 & -1.814 & -1.464 & -1.468 & - . - g & -1.649 & -1.500 & -0.149 \\
\hline M 13 & $\ldots f$ & $\ldots . e$ & -1.746 & -1.670 & -1.646 & -1.521 & -1.638 & $\ldots$ & -1.644 & -1.500 & -0.144 \\
\hline M 92 & $\ldots f$ & $\ldots e^{e}$ & -2.421 & -2.621 & -1.884 & -2.900 & -1.636 & .... & -2.292 & -2.200 & -0.092 \\
\hline
\end{tabular}

b) Field Stars ${ }^{m}$

\begin{tabular}{|c|c|c|c|c|c|c|c|c|c|c|c|}
\hline K Giants & -0.025 & $\ldots{ }^{e}$ & $\ldots h^{h}$ & $\ldots h$ & $\ldots h^{h}$ & +0.007 & -0.045 & -0.034 & $\ldots$ & $\ldots$ & -0.024 \\
\hline G Dwarfs & $\ldots i$ & $-0.006^{j}$ & -0.008 & $\ldots k$ & -0.007 & +0.004 & +0.023 & $\ldots$. & $\ldots$ & $\ldots$ & +0.001 \\
\hline
\end{tabular}

${ }^{a}$ Unweighted mean of all entries not in italics.

b $\Delta \mathrm{Fe} \equiv[\mathrm{Fe} / \mathrm{H}]_{\text {out }}-[\mathrm{Fe} / \mathrm{H}]_{\mathrm{o}}$. For field giants and dwarfs, this is the straight mean of entries on this line.

${ }^{\mathrm{c}}$ Giants only with $V-K>1.90$.

d All dwarfs, plus subgiants with $V-K>1.90$; omitted in means because of higher error.

${ }^{\mathrm{e}}$ No dwarfs available; not abundance-sensitive in giants.

${ }^{\mathrm{f}}$ Not measurable; spectra centered too far to red.

${ }^{\mathrm{B}}$ Uncertain interstellar contamination; omitted.

${ }^{\text {h }}$ No independent $[\mathrm{Fe} / \mathrm{H}]$ can be derived since $\log g$ has been force-fitted to $\mathrm{Mg}_{2}$. See text.

${ }^{i}$ Not abundance-sensitive in dwarfs.

${ }^{j}$ Stars with $V-K<2.10$ only. Errors larger for cooler stars. $Z$-fit is valid only up to $V-K=2.50$.

${ }^{k}$ Noisy abundance indicator in dwarfs; not used.

${ }^{1}$ Biased too high due to metal-poor dwarfs (see Fig. 14g); omitted.

${ }^{\mathrm{m}}$ For field stars, the entries are mean $\Delta \mathrm{Fe}$ by feature and, in the far right-hand column, averaged over all features.

TABLE 8

ERRORS IN DERIVED $[\mathrm{Fe} / \mathrm{H}]_{\text {out }}$, BY FEATURE ${ }^{\mathrm{a}}$

\begin{tabular}{|c|c|c|c|c|c|c|c|c|c|c|}
\hline Group & $\mathrm{CN}$ & G band & $\mathbf{M g}_{2}$ & $\mathbf{M g}_{1}$ & $\mathbf{M g} b$ & Fe5270 & Fe5335 & $\mathrm{Na} D$ & Net Pred. Error & Obs. Error \\
\hline K Giants & $0.180^{d}$ & $\ldots e$ & $0.250^{f}$ & $0.350^{f}$ & $0.350^{f}$ & $0.250^{g, h}$ & $0.230^{g}$ & 0.200 & 0.100 & 0.16 \\
\hline G Dwarfs & $\ldots e$ & $0.400^{i}$ & $0.250^{i}$ & $\ldots e$ & 0.530 & $0.320^{9}$ & $0.350^{g}$ & $0.320^{9}$ & 0.135 & 0.21 \\
\hline
\end{tabular}
ble 5 .

${ }^{a} \mathrm{RMS}$ error in derived $[\mathrm{Fe} / \mathrm{H}]_{\text {out }}$ averaged over all stars, except as noted. Errors for NGC 188, M71, and metal-poor clusters are higher than the rms quoted here. Values given are in dex.

${ }^{\mathrm{b}}$ Predicted rms error in average $[\mathrm{Fe} / \mathrm{H}]_{\text {out }}$, assuming feature errors add Gaussianly and with feature weights as given in Notes to Table 5 . Errors for stars with $[\mathrm{Fe} / \mathrm{H}]<-1.00$ are larger.

${ }^{c}$ Observed rms $\left([\mathrm{Fe} / \mathrm{H}]_{\text {out }}-[\mathrm{Fe} / \mathrm{H}]_{\mathrm{o}}\right)$ from stars in Table 5.

${ }^{\mathrm{d}}$ For giants with $V-K>1.90$ and $[\mathrm{Fe} / \mathrm{H}]_{\text {out }}>-1.00$, using Fit 2 in Table 6.

${ }^{\mathrm{e}}$ Insensitive to $[\mathrm{Fe} / \mathrm{H}]$; not used in $[\mathrm{Fe} / \mathrm{H}]_{\text {out }}$.

${ }^{f}$ Field $\mathrm{K}$ giants excluded, since their $\log g$ has been forced to fit $\mathrm{Mg}_{2}$, and their residuals are anomalously small.

${ }^{8}$ Errors larger below $[\mathrm{Fe} / \mathrm{H}]=-1.00$.

${ }^{\mathrm{h}}$ Derived $[\mathrm{Fe} / \mathrm{H}]_{\text {out }}$ may be systematically slightly too high for stars with $[\mathrm{Fe} / \mathrm{H}]>+0.20$.

${ }^{\mathrm{i}}$ For dwarfs with $V-K<2.10$ only, from Fit 3 in Table 6. Errors larger for $2.10 \leq V-K \leq 2.50$. 
trum with an accuracy of order \pm 0.15 dex, comparable to the accuracy of other low-resolution techniques.

\subsection{Errors in the Output Gravities}

The input vs. output gravities in Figure 15 give no evidence for any systematic error as a function of atmospheric parameters. There are only two features, $\mathrm{Mg}_{2}$ and $\mathrm{Mg} b$, that yield useful estimates of gravity. The observed rms errors for each of these based on the residuals in Figure 15 are tabulated in Table 9. Again, assuming that errors add in a Gaussian manner, we predict a net rms error in $\log g$ of $\sim 0.23$ dex based on a weighted average of the two features. This compares favorably to the observed input-output rms difference of 0.25 dex from Table 5. The close equality of these numbers may reflect the fact that the estimated input gravity errors per star are small, of order 0.1 dex. An error of 0.23 dex corresponds to $0.58 \mathrm{mag}$ in luminosity, or $30 \%$ in distance. The two $\mathrm{Mg}$ indices together are thus moderately useful indicators of luminosity and/or distance.

In this and the preceding section, we have estimated output errors in one parameter under the condition that the other parameter is precisely known. That is not the case for a random field star, for which no information is usually available a priori. It can be shown that uncertainty in gravity has a negligible effect on the derived output metallicity, especially if estimates from $\mathrm{Mg}_{2}$ and $\mathrm{Mg} b$ are deweighted slightly. Uncertainty in assumed $[\mathrm{Fe} / \mathrm{H}]$ has a large effect on output gravity, however. For example, an error of 0.1 dex in assumed metallicity translates to an error of 0.30 dex in output gravity for $\mathrm{Mg}_{2}$. This suggests that reaching the above target error of $0.23 \mathrm{dex}$ per spectrum depends on having excellent metallicity estimates. Further, an appreciable fraction of the error we observe in our output gravities may actually stem from errors in the assumed input metallicities.

\section{DISCUSSION AND SUMMARY}

We have derived empirical fitting functions for 11 atomic and molecular features that are prominent in the spectra of $G$

TABLE 9

ERRORS IN DERIVED $(\log g)_{\text {out }}$, BY FEATURE ${ }^{a}$

\begin{tabular}{ccccc}
\hline \hline Group & $\mathrm{Mg}_{2}$ & $\mathrm{Mg} b$ & $\begin{array}{c}\text { Net } \\
\text { Predicted } \\
\text { Error }^{\mathrm{b}}\end{array}$ & $\begin{array}{c}\text { Observed } \\
\text { Error }^{\mathrm{c}}\end{array}$ \\
\hline $\mathrm{K}$ giants $\ldots \ldots .$. & 0.25 & 0.35 & 0.23 & 0.25 \\
$\mathrm{G}$ dwarfs $\ldots \ldots$ & 0.33 & 0.25 & 0.22 & 0.25 \\
\hline
\end{tabular}

NotE.-Values are given in dex. For feature weights, see Notes to Table 5 .

${ }^{\mathrm{a}} \mathrm{Rm}$ s errors in derived $(\log g)_{\text {out }}$ from stars in Table 5 with independent input gravities. For giants, these are averages of NGC 188, M67, NGC 7789 , and the Hyades (all other stars yield biased values). Errors for stars with $[\mathrm{Fe} / \mathrm{H}]<-1.00$ are larger. For dwarfs, values include all stars with $(\log g)_{\text {out }}$ in Table 5.

Predicted rms error in derived $(\log g)_{\text {out }}$ assuming the two feature errors add in Gaussian fashion and with feature weights as given in notes to Table 5 .

${ }^{c}$ Observed rms error $\left[(\log g)_{\text {out }}-(\log g)_{\text {o }}\right]$ from stars in Table 5. and $\mathrm{K}$ stars. The calibration should be accurate for stars in our region of the Galaxy over the range $3-15 \times 10^{9} \mathrm{yr}$ in age and near solar in metallicity. Although the calibrating stars densely sample the range -0.75 dex to +0.5 dex in metallicity, there is a large range in calibrating gravities only close to solar. The gravity partial derivatives are therefore likely to be more uncertain well away from solar metallicity.

In particular, the present stellar sample lacks the very old, very metal-rich stars likely to be present in elliptical galaxies. The current calibration derives the metallicity dependence at very high $Z$ from the highest metallicity stars in the solar neighborhood. However, because these stars are mostly young, we must infer the gravity correction for the older ages required in ellipticals by using the gravity partial derivatives of solar metallicity stars. The same caveat applies to young metal-poor stars, which are also missing from the stellar sample. The accuracy of the gravity calibration at both high and low metallicities away from solar can and should be checked with theoretical model spectra.

For most spectral features, the derived fitting functions give no surprise: the fitting behavior closely mimics that expected from the physics of stellar atmospheres. The one exception is the index CN (4150A), for which we find only minimal evidence for traditional gravity effects but strong evidence for evolutionary mixing of $\mathrm{CNO}$ products to the stellar surface. This conclusion is important for modeling the integrated $\mathrm{CN}$ indices of external stellar populations.

To test the fits, we have derived output gravities and metallicities for each of the calibrating stars and clusters. The internal random errors for these quantities are fairly small, being \pm 0.15 dex in $[\mathrm{Fe} / \mathrm{H}]$ and \pm 0.23 dex in $\log g$ (per stellar observation) and \pm (a few hundredths of a dex $)$ for cluster metallicities. The output metallicity scale merely smooths and averages the input metallicity scale and does not offer any new information of a systematic nature. Thus the status of the scale above solar metallicity is still provisional, pending reliable high-dispersion abundance determinations for the SMR stars.

For most indices, the line-strength residuals about the final fits are marginally larger than the observational errors-just sufficiently so that one feels obligated to inquire into their origin. A variety of factors may play a role: occasional data with large observational errors, errors in the assumed input parameters, evolutionary mixing anomalies in $\mathrm{CN}$, and possible cluster contamination by nonmembers (especially in M71). Nevertheless, one feels that at least some deviations cannot be explained in these ways, notably the systematic behavior of the $\mathrm{Mg}$ indices in NGC 188, the large but seemingly patternless scatter for several indices in M71 and NGC 7789, and the systematic residuals for the same two clusters in $\mathrm{H} \beta$. Reobservations of these objects would be worthwhile.

Despite these exceptions, it is worth emphasizing that just three basic input parameters-temperature, mean metallicity, and gravity-do a remarkably good job of predicting the spectral features seen in these stars. This suggests among other things that the abundances of elements other than $\mathrm{Fe}$ are closely and predictably tied to Fe itself-the so-called lockstep hypothesis. This conclusion is also emerging strongly from high-dispersion studies of disk and halo stars in the Galaxy. These trends in other elements versus $\mathrm{Fe}$ are of course built into the present calibration process; thus the application of our 
fitting functions to features in other galaxies will test whether the same trends are obeyed there also.

We view this analysis as a first step. Further tests are planned using theoretical model spectra, more detailed comparisons to Galactic cluster stars, and observations of old, metal-rich stars in the Galactic bulge. Models of elliptical galaxy spectra using the present fitting functions are also underway.

We would like to thank Eileen Friel for her help on earlier stages of this work, Jay Frogel for unpublished $V-K$ photome- try, John Laird for providing his $\mathrm{G}$ dwarf list in advance of publication, and Ron Marzke for assistance. Guy Worthey assisted in the gravity analysis in Appendix A, and a very careful referee made numerous suggestions that greatly improved the presentation. Gerri McLellan and Pat Shand ably prepared the manuscript and tables. J. G. would like to thank Lick Observatory for its hospitality. This work was partially supported by National Science Foundation grants AST 76-08258, 82-11551, and 87-02899 and by ASU Faculty Grants-in-Aid to D. B. J. G. was supported by a fellowship from the Fundacion del Amo of the Universidad Complutense.

\section{APPENDIX A \\ ERRORS IN THE INPUT GRAVITIES AND THEIR EFFECT ON COMPUTED STELLAR POPULATION MODELS}

In this Appendix we discuss more fully the accuracies and tests we have made of the assigned cluster-star gravities and the effect of residual gravity errors on the fitting functions and computed integrated indices. We already noted in the main text $(\$ 2.3 .3)$ that we get good agreement in the gravities using different published giant-branch tracks. The rms difference between the Yale and VandenBerg and Laskarides giant branches is only 0.03 dex in $\log g$, with no systematic offset. This stability occurs because we are using luminosity (rather than surface temperature) to place stars on the giant branch. We are thus insensitive to uncertainties in the color-temperature calibration, and any remaining error (due to mismatch in the mean temperature of the track to the mean colors of the stars) is found to be less than 0.1 dex for all clusters, except NGC 188 and NGC 7789.

These latter two clusters exhibit the largest residual deviation in $B-V$ from the theoretically predicted isochrones, as shown in Figures $2 a$ and $2 b$. In NGC 188, the giants look too red by $0.15 \mathrm{mag}$, which corresponds to an error of 0.15 dex in the assigned log $g$. NGC 7789 exhibits an offset of similar magnitude but of opposite sign.

For NGC 188, Twarog (1978) first proposed a mass loss of $\sim 0.20-0.30 M_{\odot}$ to explain the red giant branch. More recently, Twarog \& Anthony-Twarog (1989) have proposed a higher reddening coupled with a younger age of $6.5 \times 10^{9} \mathrm{yr}$ for NGC 188 . The effect on our assigned gravities for even this large an age change is surprisingly small. This is due to the fact that the fitted surface temperature is always tied closely to the observed colors and to the fact that various factors tend to cancel when the distance modulus is left as a free parameter in the fit, as was done there. The net result is that the gravities of both turnoff and giant-branch stars change by only 0.03-0.07 dex using the new parameters. The even newer parameters and newer tracks used by Hobbs, Thornburn, \& Rodriguez-Bell (1990) are intermediate in character between our values and the Twarogs' and therefore yield still smaller gravity changes. The conclusion is that new evolutionary tracks, or even different fits to the same tracks, by themselves have only a small effect on the assigned $\log g$ 's. To be doubly sure of this, we have compared to published values of $\log g$ fit by other authors using similar but not quite identical methods. The largest body of overlap is the atlas of globular cluster stars by Frogel, Persson, \& Cohen (1983). Comparison using several clusters shows a mean offset of $\leq 0.1$ dex in the sense that our gravity scale may be slightly lower than theirs.

As a final check, we have referred to the compilation of field-star giant gravities assembled by McWilliam (1990). Only accurate trigonometric values (plus three Hyades giants) have been used. These external estimates are compared to our output values of log $g$ obtained by inverting the fitting functions ( see Table 5 ). For eight nearby giants, the mean difference is found to be -0.1 dex (we are again too low), but with a scatter of only $0.16 \mathrm{dex}$. This is well within the rms output error of 0.23 dex per star quoted in $\S 5$.

From these various tests, we conclude that our input gravity errors must be less than 0.2 dex for essentially all stars and that systematic errors for the gravity scale as a whole are $\leq 0.1 \mathrm{dex}$.

It is of interest to inquire the effect of such a gravity error on the integrated line strengths of a stellar population model. $\mathrm{Mg}_{2}$ is the most gravity-sensitive of our indices; contours in Figure $4 c$ show that a systematic change of 0.1 dex for an entire old population would translate to a change in integrated $\mathrm{Mg}_{2}$ of about $2 \%$, or 0.007 mag. This is a small effect.

Finally, we mention why it is possible (at least in principle) to detect small age differences among external galaxy populations, yet the fitting functions themselves are not sensitive to the precise ages or tracks assumed for the calibrating clusters. This is so because the integrated spectra of external galaxies respond primarily to the mean temperature of the stellar population, which declines as the population ages. As we have gone about deriving the fitting functions, the crucial input temperatures of the calibrating stars (actually $V-K$ ) are tied directly to observations and are independent of any assumed tracks and ages. Tracks and ages do affect the assumed gravities, but the integrated spectra of old stellar populations are much less sensitive to this parameter, and it does not have to be known so precisely.

On the other hand, it is only the fitting functions themselves that are insensitive to the tracks. This insensitivity to tracks will end the moment we actually attempt to calculate a real model galaxy. At that point, the mean temperature of the adopted population isochrone will enter as the most important single factor in calculating integrated indices. For now, however, in deriving the fitting functions themselves we are still free of this effect. 


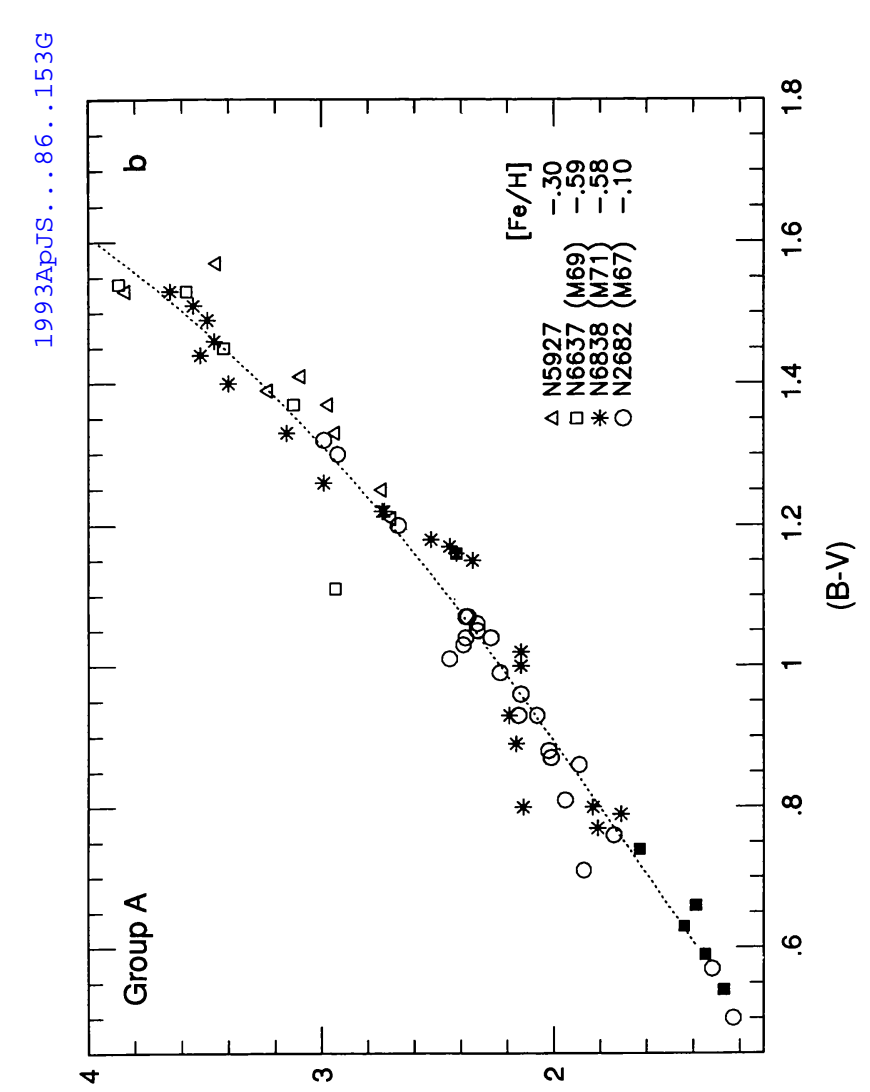

(X)-N)
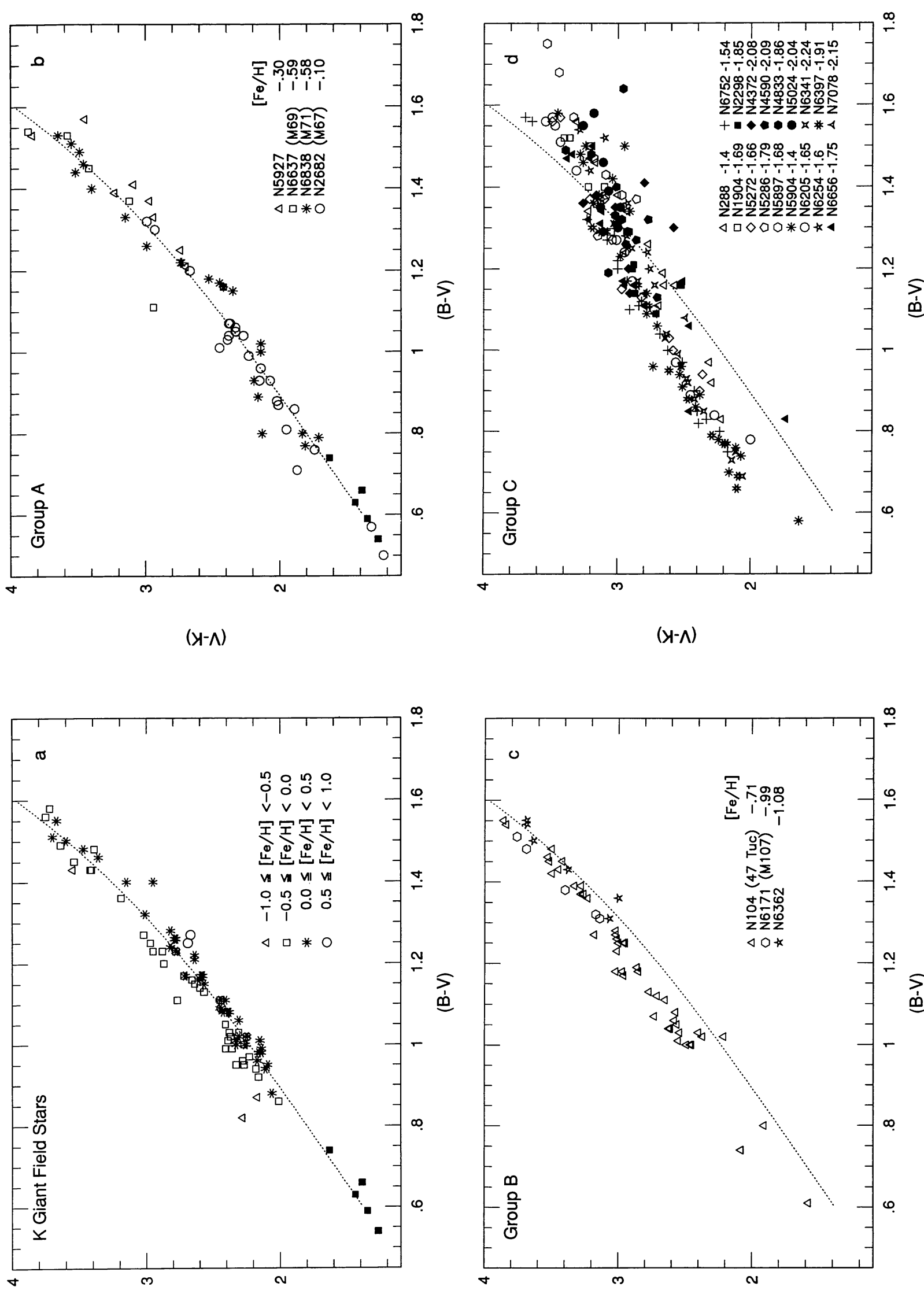

$(x-\Lambda)$

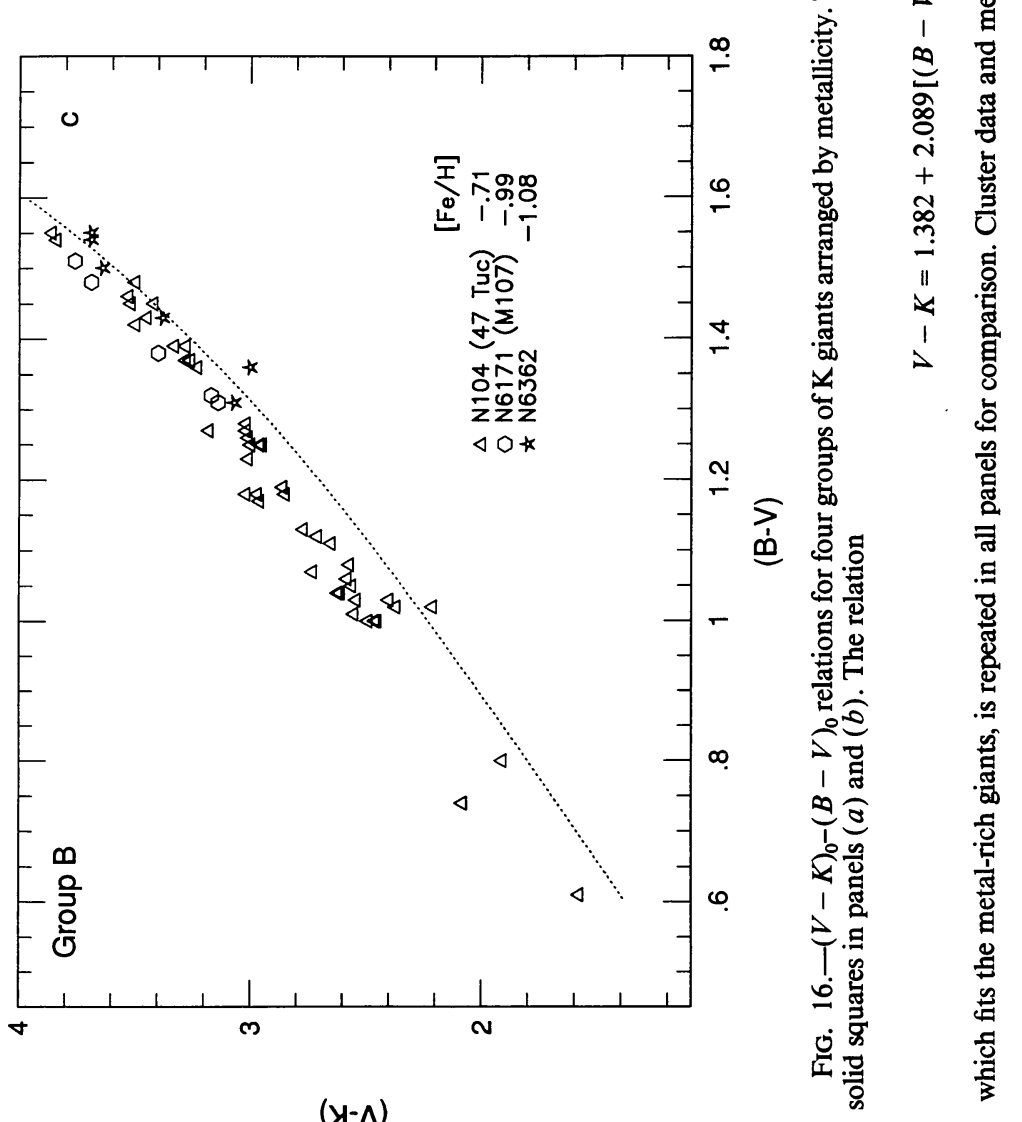




\section{APPENDIX B \\ $V-K$ VERSUS $B-V$ CALIBRATION FOR K GIANTS}

For several clusters, the lack of $K$-band observations necessitated the calculation of $V-K$ from $B-V$. The $B-V$ versus $V-K$ relation is a function of metallicity, and therefore a sample of stars covering a wide range in [ $\mathrm{Fe} / \mathrm{H}]$ is required to establish the correspondence. The field $\mathrm{K}$ giants from Paper II provide a group of stars near solar metallicity for which $V-K$ and $B-V$ are known. For lower metallicities, the reddening-corrected globular cluster observations of Frogel et al. (1983) were used. Four metallicity groups were formed: field $\mathrm{K}$ giants above $[\mathrm{Fe} / \mathrm{H}]=-0.6$, group A globulars with $-0.6 \leq[\mathrm{Fe} / \mathrm{H}] \leq-0.1$, group $\mathrm{B}$ globulars with $-1.25 \leq[\mathrm{Fe} / \mathrm{H}] \leq-0.6$, and group $\mathrm{C}$ globulars with $[\mathrm{Fe} / \mathrm{H}] \leq-1.25$. The metallicities for the globular clusters have been taken from Frogel et al. (1983).

The $B-V, V-K$ relations for each of these groups is shown in Figures $16 a-16 d$. In NGC 188, there are turnoff and subgiant stars that are hotter than the calibrating $\mathrm{K}$ giants, so it is important to check that the giants merge smoothly with the dwarf main-sequence calibration of Johnson (1966). Johnson's calibration is shown by the solid squares at the lower left of Figures $16 a$ and $16 b$. It merges satisfactorily with the $\mathrm{K}$ giants.

The relations for the field stars and group A globulars, plotted in Figures $16 a$ and $16 b$, agree well, which is not surprising since their metallicities are similar. To guide the eye, the function $V-K=1.382+2.089[(B-V)-0.6]+0.500[(B-V)-0.6]^{4}$ has been plotted as the dotted line in all panels. This fits the metal-rich giants well in Figures $16 a$ and $16 b$. The field $\mathrm{K}$ giants in panel a may show a slight systematic offset with metallicity, but this trend does not appear to continue unbroken into the group A globulars. We have not corrected for it, and in any case it is only a few hundredths of a magnitude.

Relative to this, the metal-poor giants are offset but in a rather complicated way. The locus for group B ( -0.6 to -1.25 dex ) is somewhat straighter but shallower than the dotted line and crosses it at $B-V \sim=1.5$. Group $\mathrm{C}([\mathrm{Fe} / \mathrm{H}] \leq-1.25)$ is shallower still but crosses to the blue, at $B-V \simeq 1.3$.

Attempts to model this complicated dependence with a single fitting formula failed; no simple expression could be found that matched the behavior of all groups satisfactorily. As an expedient, we simply read off a star's missing $V-K$ color from the panel with the appropriate abundance. From the scatter in Figure 16, we estimate errors of $\sim 0.15$ mag, with occasional errors up to 0.3 mag for metal-poor stars.

\section{APPENDIX C COMPARISON OF $\mathrm{Mg}_{2}$ TO MOULD'S THEORETICAL Mg INDICES}

In this Appendix, we show an example of how the fitting functions can be used to test theoretical spectral indices by comparing the fitting function for $\mathrm{Mg}_{2}$ (expression [6] in Table 6) with Mould's (1978) models for the Mg feature. Mould defined two indices, $w$ and $D$, and we compare to both. The only subtlety is defining $V-K$ from $T_{e}, \log g$, and $Z$. For this, we have used a $T_{e}$, $(V-K)$ solar-abundance relation that is a compromise among Ridgway et al. (1980), Cohen et al. (1978), and (for dwarfs) Johnson (1966). This has been corrected to other abundances using the blanketing vector for $(V-K)$ given by Taylor et al. (1987), scaled in a plausible way with temperature. However, virtually all the points plotted refer to solar abundance, so this correction makes little difference.

Figure 17 compares the fitting function estimates to the raw index values, which have been plotted logarithmically to allow for a possible scale difference between the theoretical and observed indices. The agreement is fair and is about equally good in either index if transformation is allowed. Further insight comes from inspecting the partial derivatives with $Z$ and log $g$, given in Table $A 1$.

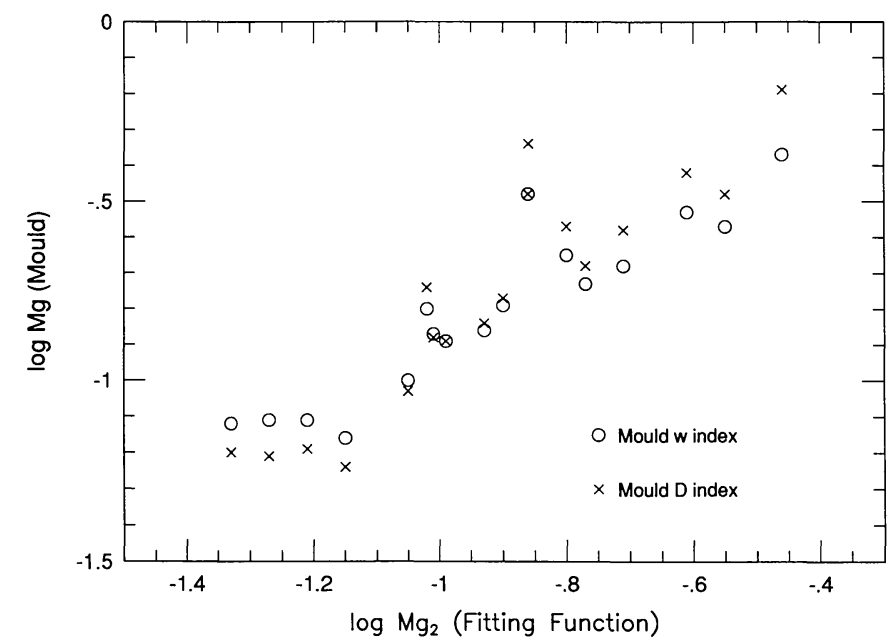

FIG. 17.-Mould (1978) synthetic $\mathrm{Mg}$ indices $w$ and $D$ vs. fitting function values for $\mathbf{M g}_{2}$ from Table 6 . 
TABLE A1

Partial Derivatives OF Mg INDICES ${ }^{a}$

\begin{tabular}{|c|c|c|c|c|c|c|}
\hline \multirow[b]{2}{*}{$T_{e}$} & \multirow[b]{2}{*}{$\log g$} & \multirow[b]{2}{*}{$Z$} & \multicolumn{2}{|c|}{ MOULD $^{\mathbf{b}}$} & \multicolumn{2}{|c|}{ FITTING FUNCTION $^{c}$} \\
\hline & & & $\log w$ & $\log D$ & Full $V-K$ & No $V-K$ \\
\hline \multicolumn{7}{|c|}{ A. Metallicity Derivatives } \\
\hline 6000 & 4.5 & -0.25 & 0.24 & 0.34 & 0.26 & 0.24 \\
\hline 5500 . & 3.5 & -0.25 & 0.23 & 0.31 & 0.34 & 0.24 \\
\hline 5500 . & 3.0 & -0.25 & 0.22 & 0.30 & 0.33 & 0.24 \\
\hline 5000 . & 3.0 & -0.25 & 0.25 & 0.32 & 0.31 & 0.25 \\
\hline 4500 . & 2.25 & -0.25 & 0.26 & 0.31 & 0.38 & 0.27 \\
\hline 4000 . & 0.75 & -0.25 & 0.10 & 0.13 & 0.42 & 0.28 \\
\hline \multicolumn{7}{|c|}{ B. Gravity Derivatives } \\
\hline 5500 & 3.0 & 0.00 & 0.23 & 0.30 & 0.13 & - \\
\hline 5000 & 3.5 & 0.00 & 0.16 & 0.20 & 0.22 & - \\
\hline 4500 & 2.0 & 0.00 & 0.16 & 0.16 & 0.13 & - \\
\hline
\end{tabular}

${ }^{a}$ Logarithmic derivative per unit dex change in $[\mathrm{Fe} / \mathrm{H}]$ or $\log g$ at the location given.

${ }^{b}$ From Mould 1978.

${ }^{\text {c } F u l l ~} V-K$ uses the full $Z$-correction to $V-K$ from Taylor et al. 1987. No $(V-K)$ omits this. The gravity derivatives are taken at fixed $V-K$ and do not involve a $Z$-correction.

We have added an extra value for our derivative that corresponds to omitting the Taylor et al. $Z$-correction to $V-K$. This shows the sensitivity to this assumption and probably brackets the real situation.

The table shows that Mould's theoretical $w$ index provides a better match to the observed partial derivatives than does $D$ if there is in fact little $Z$ dependence in $V-K$. The match to the metallicity derivative is particularly good except for the coolest point at 4000 $\mathrm{K}$, where both $w$ and $D$ are much less sensitive to $Z$ than observed in real stars. The gravity match is also good except for the hottest stars. These have weak lines, however, and the observed derivatives are not well determined.

The overall agreement is encouraging but is marred somewhat by the fact that Mould assumed $[\mathrm{Mg} / \mathrm{Fe}]=0$ throughout, whereas our $\mathrm{Mg}_{2}$ fitting function incorporates whatever $[\mathrm{Mg} / \mathrm{Fe}]$ abundance trajectory is present in local metal-rich Galactic stars. We give reasons in Worthey et al. (1992) why it is plausible that $[\mathrm{Mg} / \mathrm{Fe}]$ in fact is 0 for the calibrating stars, but uncertainty clouds somewhat the present comparison.

\section{REFRENCES}

Bell, R. A., \& Gustafsson, B. 1989, MNRAS, 236, 653

Bond, H. E., Faber, S. M., Burstein, D., \& Luck, R. E. 1985, ApJ, 90, 1349

Brodie, J., Huchra, J., \& Kent, S. 1991, ApJ, 370, 495

Brown, J. A. 1987, ApJ, 317, 701

Burstein, D., Faber, S. M., Gaskell, C. M., \& Krumm, N. 1984, ApJ, 287, 586 (Paper I)

Burstein, D., Faber, S. M., \& Gonzalez, J. J. 1986, AJ, 91, 1130 (Paper III)

Burstein, D., \& McDonald, L. H. 1975, AJ, 80, 17

Buzzoni, A. 1989, ApJS, 71, 817

Cacciari, C., \& Freeman, K. C. 1983, ApJ, 286, 185

Carbon, D. F., Langer, G. E., Butler, D., Kraft, R. P., Suntzeff, N. B., Kemper, E., \& Trefzger, C. F. 1982, ApJS, 49, 207

Carney, B. W. 1983a, AJ, 88, 610 1983b, AJ, 88, 623

Carney, B. W., \& Aaronson, M. 1979, AJ, 84, 867

Cayrel de Strobel, G., Bentolila, C., Hauck, B., \& Curchod, A. 1980 A\&AS, 41,405

Cayrel de Strobel, G., Bentolila, C., Hauch, B., \& Duquennoy, A. 1985, A\&AS, 59, 145

Clegg, R. E. S., Lambert, D. C., \& Tomkin, J. 1981, ApJ, 250, 262

Cohen, J. G. 1973, ApJ, 186, 149

- 1974, ApJ, 194, 37

. 1975, ApJ, 197, 117

1977, ApJ, 214, 86
Cohen, J. G. 1978, ApJ, 223, 487

. 1979, ApJ, 227, 499

1980, ApJ, 241, 981

Cohen, J. G., Frogel, J. A., \& Persson, S. E. 1978, ApJ, 222, 165

Cudworth, K. M. 1985, AJ, 90, 65

Dickow, P., Gyldenkerne, K., Hansen, L., Jacobsen, P.-U., Johansen, K. T., Kjaergaard, P., \& Olsen, E. H. 1970, A\&AS, 2, 1

Eggen, O. J. 1985, AJ, 90, 333

Eggen, O. J., \& Sandage, A. R. 1964, ApJ, 140, 130

. 1969, ApJ, 158, 669

Faber, S. M. 1973, ApJ, 179, 731

Faber, S. M., Friel, E. D., Burstein, D., \& Gaskell, C. M. 1985, ApJS, 57, 711 (Paper II)

Frogel, J. A., Persson, S. E., \& Cohen, J. G. 1979, ApJ, 227, 499 1983, ApJS, 53, 713

Gehren, T. 1988, Rev. Mod. Astron., 1, 52

Gilroy, K. K., Sneden, C., Pilachowski, C. A., \& Cowan, J. J. 1990, ApJ, 327, 298

Gilroy, K. K., \& Brown, J. A. 1991, ApJ, 371, 578

Gliese, W. 1969, Catalogue of Nearby Stars, Veroff. Astr. Rechen-Inst., Heidelberg, No. 22

Gottlieb, D. J., \& Bell, R. A. 1971, A\&A, 19, 434

Green, E. M., Demarque, P., \& King, C. R. 1987, The Revised Yale Isochrones and Luminosity Functions (New Haven: Yale Univ. Obs.) 
Greenstein, J. L., \& Oinas, V. 1974, ApJ, 193, L91

Griffin, R. A. 1968, A Photometric Atlas of Arcturus (Cambridge: Cambridge Phil. Soc.)

Griffin, R. A., \& Griffin, R. 1979, A Photometric Atlas of the Spectrum of Procyon (Cambridge: Inst. of Astronomy)

Gulati, R. K., Malagnini, M. L., \& Morossi, C. 1991, A\&A, 247, 447

$\operatorname{lil}_{I \rightarrow 1}$ Gustafsson, B., Kjaergaard, P., \& Anderson, S. 1974, A\&A, 34, 99

Hansen, L., \& Kjaergaard, P. 1971, A\&A, 15, 123

Harris, W. E., Racine, R., \& de Roux, J., 1976, ApJS, 31, 13

Hearnshaw, J. B. 1974a, A\&A, 34, 263

1974b, A\&A, 36, 191

1976a, A\&A, 51, 71

- 1976b, A\&A, 51, 85

Hobbs, L. M., Thorburn, J. A., \& Rodriguez-Bell, T. 1990, AJ, 100, 710

Hoffleit, D. 1982, The Bright Star Catalogue (4th ed.; New Haven: Yale Univ. Obs.)

Janes, K. A. 1975, ApJS, 29, 161

. 1977, AJ, 82, 35

Johnson, H. L. 1965, ApJ, 141, 170

. 1966, ARA\&A, 4, 193

Johnson, H. L., \& Sandage, A. R. 1956, ApJ, 124, 379

Johnson, H. L., Mitchell, R. I., Iriarte, B., \& Wiśniewski, W. Z. 1966, Comm. Lunar Planet. Lab., 4, 99

Johnson, H. L., MacArthur, J. W., \& Mitchell, R. I. 1968, ApJ, 152, 465

Kraft, R. P. 1979, ARA\&A, 17, 309

Kurucz, R. 1992, private communication

Laird, J. B. 1985, ApJS, 57, 389

Lambert, D. L. 1987, JA\&A, 8, 103

McWilliam, A. 1990, ApJS, 74, 1075

Mathieu, R. D., \& Latham, D. W. 1986, AJ, 90, 1364

McClure, R. D., \& van den Bergh, S. 1968, AJ, 73, 313

McClure, R. D., \& Twarog, B. A. 1977, ApJ, 214, 111

McNamara, B. J., \& Solomon, S. 1981, A\&AS, 43, 337

Mould, J. 1978, ApJ, 220, 434

Mould, J., \& McElroy, D. 1978, ApJ, 220, 580

Norris, J. 1986, in IAU Symp. 126, The Harlow Shapley Symposium in Globular Cluster Systems in Galaxies, ed. J. E. Grindlay \& A. G. Davis Philip (Dordrecht: Reidel), 93
Osborn, W. 1973, ApJ, 186, 725

Peterson, R. C. 1981, ApJS, 45, 421

Peterson, R. C., \& Carney, B. W. 1979, ApJ, 231, 762

Ridgway, S. T., Joyce, R. R., White, N. M., \& Wing, R. F. 1980, ApJ, 235, 126

Robinson, L., \& Wampler, E. J. 1972, PASP, 84, 161

Sanders, W. L. 1977, ApJS, 27, 89

Spinrad, H., \& Taylor, B. J. 1969, ApJ, 157, 1279

Sweigart, A. V. 1987, ApJS, 65, 95

Taylor, B. J. 1980, AJ, 85, 242 1986, ApJS, 60, 577

Taylor, B. J., Johnson, S. B., \& Joner, M. D. 1987, AJ, 93, 1253

Trefzger, C. F., Carbon, D. F., Langer, G. E., Suntzeff, N. B., \& Kraft, R. P. 1983, ApJ, 266, 144

Tripicco, M. J. 1989, AJ, 97, 735

Tripicco, M. J., \& Bell, R. A. 1990, AJ, 99, 691

1991, AJ, 102, 744

. 1992, AJ, 103, 1285

Twarog, B. A. 1978, ApJ, 220, 890

Twarog, B. A., \& Anthony-Twarog, B. J. 1989, AJ, 97, 759

Twarog, B. A., \& Tyson, N. 1985, AJ, 990, 1247

Upgren, A. R., Mesrobian, W. S., \& Kerridge, S. J. 1972, AJ, 77, 74

VandenBerg, D. A., 1983, ApJS, 51, 29 . 1985, ApJS, 58, 711

VandenBerg, D. A., \& Bridges, T. J. 1984, ApJ, 278, 679

VandenBerg, D. A., \& Laskarides, P. G. 1987, ApJS, 64, 103

VandenBerg, D. A., Hartwick, F. D. A., Dawson, P., \& Alexander, D. R. 1983, ApJ, 266, 747

Veeder, G. J. 1974, AJ, 79, 1056

Wallerstein, G. 1961, ApJS, 6, 407

Wheeler, J. C., Sneden, C., \& Truran, J. W., Jr. 1989, ARA\&A, 27, 279

Williams, P. M. 1971a, MNRAS, 153, 171 1971b, MNRAS, 155, 215

Worthey, G., Faber, S. M., \& Gonzalez, J. J. 1992, ApJ, 398, 69 